\title{
Synthesis of amine-functionalized heparin oligosaccharides for the investigation of carbohydrate-protein interactions in microtiter plates
}

Susana Maza, Giuseppe Macchione, Rafael Ojeda, Javier López-Prados, Jesús Angulo, José L. de Paz,* Pedro M. Nieto*

Glycosystems Laboratory, Instituto de Investigaciones Químicas (IIQ), Centro de Investigaciones Científicas Isla de La Cartuja, CSIC and Universidad de Sevilla, Américo Vespucio, 49, 41092 Sevilla, Spain.

* Corresponding author. Fax: +34 954 460565; e-mail: jlpaz@iiq.csic.es

\begin{abstract}
The synthesis of well-defined oligosaccharides is crucial for the establishment of structure-activity relationships for specific sequences of heparin, contributing to the understanding of the biological role of this polysaccharide. It is highly convenient that the synthetic oligosaccharides contain an orthogonal functional group that allows selective conjugation of the probes and expands their use as chemical tools in glycobiology. We present here the synthesis of a series of amine-functionalized heparin oligosaccharides using a $n+2$ modular approach. The conditions of the glycosylation reactions were carefully optimized to produce efficiently the desired synthetic intermediates with an $\mathrm{N}$-benzyloxycarbonyl-protected aminoethyl spacer at the reducing end. The use of microwave heating greatly facilitates $O$ - and $N$-sulfation steps, avoiding experimental problems associated with these reactions. The synthesized oligosaccharides were immobilized in 384-well microtiter plates and successfully probed with a heparin-binding protein, the basic fibroblast growth factor FGF-2. The use of hexadecyltrimethylammonium bromide minimized the amount of sugar required
\end{abstract}


for attachment to the solid support. Using this approach we quantified heparin-protein interactions, and surface dissociation constants for the synthetic heparin derivatives were determined.

\section{Introduction}

Glycosaminoglycans (GAGs) are natural, linear polysaccharides that in general present a high level of sulfation. This family of molecules includes heparin, heparan sulfate, and chondroitin sulfate, among others. GAGs are biosynthesized in the Golgi apparatus of eukaryotic cells through the sequential action of a series of enzymes. This natural process results in GAG chains with a high level of structural diversity. The heterogeneity of GAG polysaccharides enables their binding to a wide range of proteins. ${ }^{1-5}$ As a consequence of these interactions, GAGs influence many biological processes such as inflammation, angiogenesis and blood coagulation. ${ }^{6}$ However, the GAG structural requirements for binding and mediating biological activity have been only established for a few cases, the most studied example being the interaction of antithrombin with heparin. ${ }^{7}$ In many other cases, the encoded information contained in specific GAG sequences is still poorly understood. This fact can be explained by the difficulties to obtain well-defined, homogeneous GAG oligosaccharides from natural sources, due to the structural complexity of these molecules.

In this context, chemical synthesis appears as an alternative, powerful tool to access well-defined GAG oligosaccharides ${ }^{8,9}$ for the determination of structure-activity relationships and the correlation of specific sequences and sulfation patterns with protein binding and biological activity. ${ }^{10,11}$ Furthermore, chemical synthesis can provide unnatural GAG sequences and analogues, as potential tools to modulate GAGprotein interactions. 
It is highly desirable that synthesized structures contain an orthogonal functional group, with unique reactivity, for further selective conjugation of the probes. ${ }^{12}$ The incorporation of such a group, usually at the reducing end of the oligosaccharide, opens the way for a wide variety of valuable experiments in glycobiology. For example, orthogonally functionalized sugars can be selectively immobilized on glass surfaces to create carbohydrate microarrays for the high-throughput screening of interactions, ${ }^{13}, 14$ or selectively conjugated to multivalent scaffolds to generate heparin/GAG mimetics. ${ }^{15-}$ ${ }^{17}$ The protecting group that masks this orthogonal functionality should be compatible with the complex protecting-group strategy required for the synthesis of heparin oligosaccharides.

Since the pioneering synthesis of the antithrombim III binding heparin pentasaccharide, ${ }^{18}$ several synthetic approaches for heparin have been described, ${ }^{19-31}$ including polymer-supported strategies, ${ }^{32-35}$ preactivation-based one-pot synthesis, ${ }^{36}$ and reactivity-based one-pot synthesis. ${ }^{37}$ Despite all these significant advances in the field, the synthesis of these molecules is still a challenging task. Various difficulties are associated with these syntheses due to the complex structure of heparin and the great diversity of native sequences. A careful design of an appropriate set of protecting groups is required for the introduction of sulfate groups on selected hydroxyl and amino functions and for the stereoselective construction of glycosidic bonds. These protecting groups can have a profound effect on the efficiency of glycosylation reactions that sometimes is not easily predictable. On the other hand, deprotection/sulfation steps may require more effort and time than the assembly of the protected intermediates. These final steps, particularly sulfation reactions, on valuable and elaborated compounds, often lead to a significant decrease in isolated overall yield. 
We report here the preparation of a series of heparin oligosaccharides (one di-, one tetra- and two hexamers) that contain the $\mathrm{GlcNSO}_{3}\left(6-\mathrm{OSO}_{3}\right)-\mathrm{IdoA}\left(2-\mathrm{OSO}_{3}\right)$ repeating unit of the major sequence of heparin. An aminoethyl spacer was placed at the reducing end of the structures. A careful optimization of the reaction conditions was necessary for the efficient glycosylation of building blocks containing an $\mathrm{N}$-benzyloxycarbonylprotected amino spacer. The use of microwave irradiation facilitated $O$ - and $N$-sulfation steps, reducing reaction times and increasing isolated yields. ${ }^{38}$ The reported experimental protocol adds valuable knowledge to the existing data on heparin-like oligosaccharide synthesis. Furthermore, the synthesized oligosaccharides were immobilized in appropriate microtiter plates by using the anomeric amino group. The resulting heparin arrays were employed to measure, qualitative- and quantitatively, sugar-protein interactions. We demonstrated the utility of this system by studying the carbohydrate affinity of a well-characterized heparin-binding protein, the basic fibroblast growth factor FGF-2 that is implicated in angiogenesis, cell growth and differentiation.

\section{Results and discussion}

\section{Synthesis of amine-functionalized heparin oligosaccharides}

For the synthesis of these orthogonally functionalized oligosaccharides, we followed a stereoselective $\mathrm{n}+2$ modular approach that utilized disaccharide $\mathbf{4}$ as key building block (Scheme 1). $\mathbf{4}$ was prepared by glycosylation of trichloroacetimidate $\mathbf{1}^{26}$ and diol $\mathbf{2}^{39}$ followed by pivaloylation at position 2 , desilylation and anomeric activation, according to the synthetic strategy developed by Martín-Lomas and co-workers. ${ }^{40}$ Interestingly, lactone $\mathbf{5}$ was systematically detected in the desilylation and trichloroacetimidation reactions. ${ }^{41}$ This side product resulted from intramolecular transesterification of the methyl ester group with the free anomeric hydroxyl group and could be totally removed 
by silica gel chromatography at a later stage. Then, we introduced a protected aminoethyl spacer at the anomeric position. The choice of the amino protecting group is a crucial point of the synthetic scheme. The yields of glycosylation reactions are significantly lower when using benzyloxycarbonyl amino linkers as reported by Seeberger and co-workers ${ }^{20}$ and recently indicated by Boons and co-workers. ${ }^{21}$ An alternative $N$-(benzyl)benzyloxycarbonyl-amino linker has been successfully considered to avoid the deactivating effect of the $-\mathrm{NH}$ group. However, in preliminary experiments on a model monosaccharide, we found that the use of $N$-(benzyl)benzyloxycarbonyl amino spacer did not provide the target compound (Scheme 2). Final hydrogenolysis over $\mathrm{Pd}(\mathrm{OH})_{2}$ proceeded sluggishly and gave a complex mixture of compounds. Interestingly, Boons and co-workers reported a similar difficulty in the one-step hydrogenation of a heparin hexasaccharide containing benzyl ethers and a $\mathrm{N}$ (benzyl)benzyloxycarbamate, although they could solve this problem by using a twostep procedure involving hydrogenation over $\mathrm{Pd} / \mathrm{C}$ and then over $\mathrm{Pd}(\mathrm{OH})_{2} .{ }^{21}$ On the other hand, hydrogenolysis of monosaccharide $\mathbf{6}$ gave cleanly the fully deprotected compound 7. For this reason, we decided to employ $N$-benzyloxycarbonyl aminoethanol to protect the reducing end of our synthetic structures. Thus, $\mathbf{4}$ was efficiently transformed into acceptor 9 (Scheme 1).

We then investigated the glycosylation between $\mathbf{4}$ and $\mathbf{9}$ under standard conditions (table 1 , entry 1 ) using catalytic TMSOTf as promoter at $0^{\circ} \mathrm{C}$, but no target tetrasaccharide was isolated. The acceptor $\mathbf{9}$ and the hydrolized donor $\mathbf{3}$ were recovered from the reaction mixture. We found that addition of further quantities of promoter (up to $30 \mathrm{~mol} \%$ with respect to the donor) was necessary to obtain tetrasaccharide $\mathbf{1 0}$ in good yield (table 1, entry 4). Glycal 11 was detected as side product in the experiments carried out with large amounts of TMSOTf (table 1, entries 2-4). Treatment of 
tetrasaccharide $\mathbf{1 0}$ with hydrazine monohydrate in a pyridine/acetic acid solution followed by coupling with $\mathbf{4}$ afforded hexasaccharide $\mathbf{1 3}$ that was subsequently delevulinated $(\rightarrow \mathbf{1 4}$, Scheme 3$)$.

With the oligosaccharides $\mathbf{9 , 1 2}$ and $\mathbf{1 4}$ at hand, we initially planned the benzylation reaction of OH-4 under neutral conditions in order to introduce the required permanent protecting group at this position of the non-reducing end. Treatment of 9 with $\mathrm{BnBr}$ and silver oxide in DMF gave the fully protected disaccharide 15 although in low $25 \%$ yield (Scheme 4). ${ }^{42}$ Starting material and two side products, one resulting from the $\mathrm{N}$ benzylation of the carbamate linker and a second one derived from $\mathrm{H}-5$ iduronic acid elimination reaction ${ }^{43}$ with concomitant cleavage of the GlcN-IdoA glycosidic bond, were detected in the reaction mixture by mass spectrometry and NMR. Attempts of consuming 9 with longer reaction times resulted in an increase of byproduct formation and lower yields of target $\mathbf{1 5}$. Therefore, an alternative approach was considered to install a permanent benzyl group at position 4 of the non-reducing end of the oligosaccharides involving glycosydation with capped disaccharide 18 (Scheme 5). Coupling of monosaccharide $\mathbf{1 6}^{19,26}$ and diol $\mathbf{2}$, followed by pivaloylation at position 2 , desilylation and trichloroacetimidate activation gave 18. Then, glycosylation of 9 with 18 afforded tetrasaccharide 19 in moderate yield (Scheme 5). A considerable amount $(45 \%)$ of unreacted starting material was recovered from the reaction mixture. Surprisingly, analogous coupling between 12 and 18 failed to provide the corresponding hexasaccharide $\mathbf{2 0}$ under the reaction conditions previously optimized for the preparation of similar oligosaccharides (Table 2, entry 1). This reaction was also unsuccessful at room temperature, using the inverse procedure ${ }^{44}$ and lowering the amount of TMSOTf (Table 2, entries 2-4). In all cases, the acceptor was partly 
recovered and only traces of the desired hexasaccharide were detected in the crude mixtures by mass spectrometry.

Poor yields of glycosylations involving NH-benzyloxycarbonyl containing sugars can be associated with those obtained with $\mathrm{N}$-acetylglucosamine derivatives.

Bonnaffé and co-workers reported the failure of a glycosylation between a 2azidoglucose trichloroacetimidate disaccharide donor and a range of disaccharide acceptors containing an $\mathrm{N}$-acetyl group. ${ }^{45}$ They demonstrated that the presence of the NHAc group, even at a position remote from the reactive $\mathrm{OH}$ acceptor, inhibits the coupling. The low reactivity of the 4-hydroxy group of $\mathrm{N}$-acetylglucosamine acceptors and the poor yields associated with glycosylations at this position have long been known. Several explanations have been proposed for this general trend in preparative carbohydrate chemistry. It has been demonstrated the participation of the NHAc group in intra- and intermolecular hydrogen bonds that decrease the reactivity of the $\mathrm{OH}$ group. ${ }^{46}$ Alternatively, poor yields of couplings of $\mathrm{N}$-acetyl containing carbohydrates also result from the formation of stable glycosyl imidate side products. ${ }^{47}$ These intermediates, proposed as the kinetic products of a glycosylation reaction, derive from the nucleophilic attack of the oxygen of the NHAc group to the oxocarbenium cation. Auzanneau and co-workers reported successful glycosylations at $\mathrm{OH}-4$ of $\mathrm{N}$ acetylglucosamine using an excess (2 equiv.) of a mild Lewis acid such as $\mathrm{BF}_{3} \cdot \mathrm{Et}_{2} \mathrm{O}$ at elevated temperatures. ${ }^{48-50}$ They hypothesized that 1 equiv. of $\mathrm{BF}_{3} \cdot \mathrm{Et}_{2} \mathrm{O}$ interacts noncovalently with the nucleophilic $N$-acetyl group and the second one promotes coupling. We decided to employ these conditions for the coupling between 12 and 18. We envisioned that the excess of $\mathrm{BF}_{3} \cdot \mathrm{Et}_{2} \mathrm{O}$ would reduce the nucleophilicity of the $N$ benzyloxycarbonyl group. Gratifyingly, coupling between 12 and 18 with 1.5 equiv. of $\mathrm{BF}_{3} \cdot \mathrm{Et}_{2} \mathrm{O}$ at room temperature gave the target hexasaccharide $\mathbf{2 0}$ in acceptable yield 
(Table 2, entry 5). A larger excess of donor or the application of the inverse procedure ${ }^{44}$ did not significantly improve the yield (Table 2, entries 6-7). As long as sugar derivatives withstand treatment with multiple equivalents of Lewis acid, these conditions seem an attractive and practical alternative to couple unreactive building blocks containing $N$-benzyloxycarbonyl groups.

After preparing the fully protected oligosaccharides $\mathbf{1 5}, \mathbf{1 9}$ and $\mathbf{2 0}$, we carried out the deprotection and sulfation steps to produce the final amine-terminated probes. The use of microwave irradiation greatly facilitated $O$-sulfations, avoiding experimental problems associated with this chemical transformation such as long reaction times and poor isolated yields. ${ }^{38}$

Treatment of $\mathbf{1 5}$ with lithium hydroperoxide and then $\mathrm{NaOH}$ hydrolised acyl and methyl ester groups. Then, $O$-sulfation using $\mathrm{SO}_{3} \cdot \mathrm{Me}_{3} \mathrm{~N}$ in DMF at $100^{\circ} \mathrm{C}$ under microwave heating gave the corresponding di- $O$-sulfated disaccharide in excellent yield. Reduction of the azido group using Staudinger conditions followed by $N$-sulfation using $\mathrm{SO}_{3} \cdot \mathrm{Py}$, and hydrogenolysis to remove benzyl and carbamate groups yielded the desired disaccharide 21 in excellent $45 \%$ isolated yield from $\mathbf{1 5}$ (Scheme 6), averaging 85\% yield per step.

Starting from tetrasaccharide 19, saponification followed by microwave-assisted $O$ sulfation, Staudinger reduction, $N$-sulfation and hydrogenolysis afforded tetrasaccharide 22 in $36 \%$ yield over five steps (Scheme 6). We also performed the deprotection and sulfation of hexasaccharide $\mathbf{1 4}$ to obtain a non-natural heparin-like oligosaccharide that contains an additional $O$-sulfate group at position 4 of the non-reducing terminus, not presented in heparin. The removal of methoxycarbonyl and acyl groups was performed on 14 with lithium hydroperoxide and then $\mathrm{NaOH}$ (Scheme 7). The resulting saponified hexasaccharide was $O$-sulfated by treatment with $\mathrm{SO}_{3} \cdot \mathrm{Me}_{3} \mathrm{~N}$ complex under microwave 
irradiation and submitted to Staudinger reduction. At this point, conventional $\mathrm{N}$ -

sulfation with $\mathrm{SO}_{3} \cdot$ Py complex in a mixture $\mathrm{Et}_{3} \mathrm{~N} /$ pyridine at room temperature afforded a complex mixture of partially sulfated hexasaccharides. Fortunately, the same reaction proceeded smoothly with microwave heating, using $\mathrm{SO}_{3} \cdot \mathrm{Me}_{3} \mathrm{~N}$ in $\mathrm{DMF} / \mathrm{Et}_{3} \mathrm{~N}$ at $60^{\circ} \mathrm{C}$, to afford the $N$-sulfated intermediate in excellent yield after simple gel filtration. To the best of our knowledge, this is the first example of microwave-assisted sulfation of an amine group. These results highlight the potential of microwaves to overcome practical problems presented during sulfation steps. Final hydrogenation afforded deprotected hexasaccharide 23. Similarly, the deprotection/sulfation sequence was carried out on fully protected $\mathbf{2 0}$ to give target hexasaccharide $\mathbf{2 4}$ (Scheme 7).

\section{Binding studies in microtiter plates}

The amine-functionalized heparin-like oligosaccharides 21-24 were immobilized in Nunc Immobilizer Amino ${ }^{\mathrm{TM}}$ microtiter plates. Monosaccharide 7 was used as negative control. We employed 384-well plates to minimize the volume of sugar solutions required for immobilization. Oligosaccharides were dissolved in sodium bicarbonate buffer $(50 \mathrm{mM}, \mathrm{pH} 9.6)$ and incubated overnight at room temperature in the microtiter plate wells. During optimization of this protocol it was found that the addition of hexadecyltrimethylammonium bromide in the immobilization buffer, at 100-fold higher concentration than the carbohydrate, greatly reduced the sugar concentration required for efficient attachment. The use of this ammonium salt has been previously reported to improve the efficiency of the attachment of heparin oligosaccharides to SPR gold chips. ${ }^{15,51}$ We hypothesized that electrostatic repulsion between highly sulfated oligosaccharides that could hamper their coupling to a solid support is reduced in the presence of positively charged micelles, facilitating the immobilization of these compounds in the wells. In this way, as low as $10 \mu \mathrm{M}$ concentration of sugar in 
immobilization buffer containing $1 \mathrm{mM}$ of hexadecyltrimethylammonium bromide resulted in adequate attachment to study binding events in the wells.

After immobilization and subsequent quenching with ethanolamine, the binding of the attached compounds to a model heparin-binding protein, FGF-2, was studied. The binding assay involved incubation with the protein, followed by incubation with antiFGF polyclonal and fluorescently labelled secondary antibodies. Bound protein was detected by using a standard fluorescence microplate reader. No binding of the antibodies to wells without FGF-2 was observed. Fluorescence signals at hexa- and tetrasaccharide positions (Figure 1) were observed. FGF-2 bound to disaccharide 21 with the lowest affinity while no interaction was detected for disulfated monosaccharide 7. These results are in good agreement with the minimal structural requirements of GAGs to bind FGF-2. ${ }^{20,52-54}$ Interestingly, the short length of the C-2 amino spacer did not negatively influence sugar recognition.

Figure 1 shows a one-step qualitative analysis using only one protein concentration. This experiment gives information to differentiate compounds that interact strongly (22, 23, 24), weakly (21) or not at all (7) with FGF-2. However, classification of relative binding strengths of hexasaccharides $\mathbf{2 3}$ and $\mathbf{2 4}$ and tetrasaccharide 22 from this experiment can be inaccurate. Ranking of relative binding affinities requires the quantification of the interactions by using different protein concentrations. ${ }^{55,56}$ Thus, oligosaccharides 22, 23 and 24 were incubated with 9 concentrations of FGF-2, ranging from 581 to $3 \mathrm{nM}$. For each sugar, average fluorescence intensities of five replicate wells were plotted against protein concentration (Figure 2). Assuming that the system reached equilibrium during incubation, the curves were analyzed as Langmuir isotherms to determine surface dissociation constants $\left(\mathrm{K}_{\mathrm{D} \text {, surf }}\right) .{ }^{55}$ The best fit was obtained with a

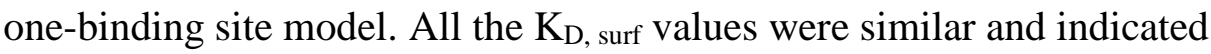


oligosaccharide binding to FGF-2 in the nanomolar range. The $\mathrm{K}_{\mathrm{D} \text {, surf values for }}$ hexasaccharides 24 and 23 were close, $32 \pm 4$ and $44 \pm 7 \mathrm{nM}$ respectively, suggesting that the presence of a non natural sulfate group at position 4 of the non reducing end of 23 did not significantly affect binding. A $K_{D}$, surf value of $68 \pm 11 \mathrm{nM}$ was determined for 22, indicating a slight reduction in affinity compared to the hexasaccharides. These values were consistent with previous measurements of heparin-FGF2 binding affinities. ${ }^{57}$ These results show that the apparent binding strength of heparin-protein interactions can be quantitatively analyzed by this method with small amounts of carbohydrate and protein and using standard microplate equipment.

\section{Conclusions}

In summary, we have reported several practical improvements that facilitate the production of amine-functionalized heparin-like oligosaccharides. We have demonstrated that the difficulties encountered in glycosylations involving $\mathrm{N}$ benzyloxycarbonyl containing building blocks can be overcome by the use of an excess of $\mathrm{BF}_{3} \cdot \mathrm{Et}_{2} \mathrm{O}$. These couplings are often associated with poor yields due to the nucleophilicity of the $\mathrm{NH}$ group. We have shown that the application of these reaction conditions can solve this problem as long as donor and acceptor are stable under these harsh conditions. On the other hand, microwave heating increased the yield and efficiency of $O$ - and $\mathrm{N}$-sulfations. The use of microwave irradiation avoided some problems related to the complete sulfation of oligosaccharides containing multiple $\mathrm{OH}$ and $\mathrm{NH}_{2}$ groups. Moreover, we have developed an approach to study interactions between these synthetic oligosaccharides and proteins in microtiter plates. Highly negatively charged sugars were efficiently attached to the solid surface with the help of positively charged hexadecyltrimethylammonium bromide. The binding of the immobilized oligosaccharides to FGF-2 was analized qualitative- and quantitatively by 
using standard microplate equipment, with minimal consumption of sugar $(0.2$

$\mathrm{nmol} /$ well) and protein (pmol/well). The presented approach provides useful

information for the preparation of heparin oligosaccharides and the evaluation of their interactions with proteins that are crucial aspects to improve our understanding of the biological role of heparin at the molecular level.

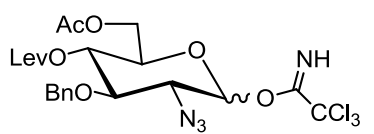

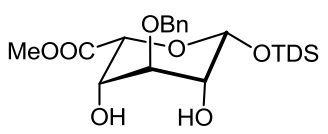

TMSOTf, $\mathrm{CH}_{2} \mathrm{Cl}_{2}-30^{\circ} \mathrm{C}$

ii) PivCl, DMAP, Py, $46 \%$, two steps

iii) $(\mathrm{HF})_{n} \cdot \mathrm{Py}, \mathrm{THF}, 68 \%$

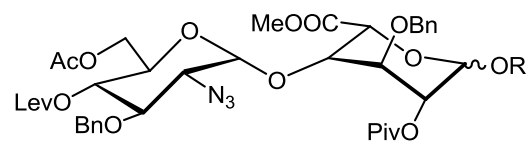

$\mathrm{Cl}_{3} \mathrm{CCN}, \mathrm{K}_{2} \mathrm{CO}_{3}, \mathrm{CH}_{2} \mathrm{Cl}_{2}, 88 \%\left(\begin{array}{l}\text { 3: } \mathrm{R}=\mathrm{H} \\ 4: \mathrm{R}=\mathrm{C}(\mathrm{NH}) \mathrm{CCl}_{3}\end{array}\right.$

$\mathrm{HO}\left(\mathrm{CH}_{2}\right)_{2} \mathrm{NHCO}_{2} \mathrm{Bn}, \mathrm{TMSOTf}$

$\mathrm{CH}_{2} \mathrm{Cl}_{2}, 0^{\circ} \mathrm{C}, 77 \%$

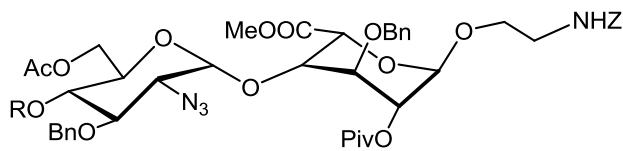

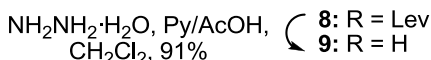

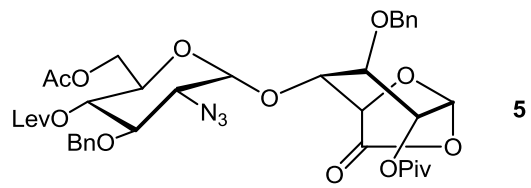

Scheme 1. Synthesis of disaccharide building blocks. 


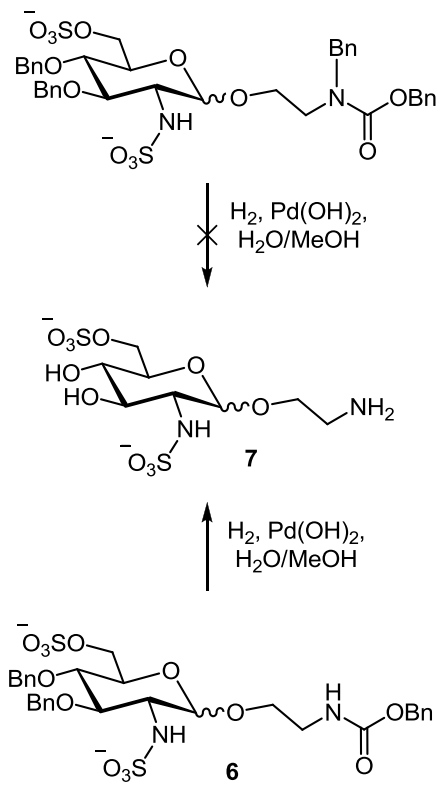

Scheme 2. Previous experiments with glucosamine monosaccharides.

Table 1. Reaction conditions for the glycosylation between $\mathbf{4}$ and 9

Isolated
Entry




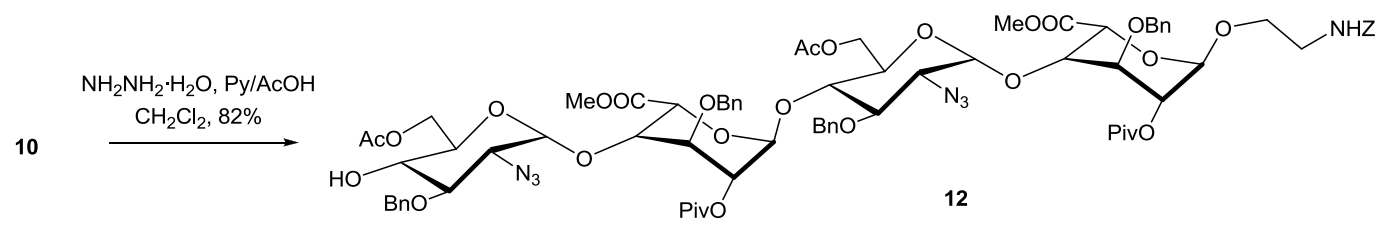

4, TMSOTf, $\mathrm{CH}_{2} \mathrm{Cl}_{2}, 0^{\circ} \mathrm{C}, 61 \%+28 \% 12$

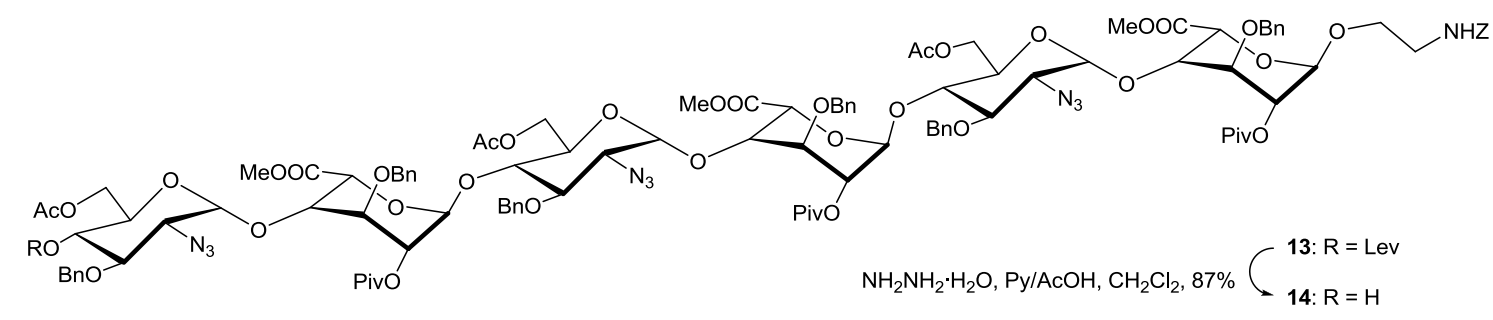

Scheme 3. Synthesis of hexasaccharide intermediate 14.

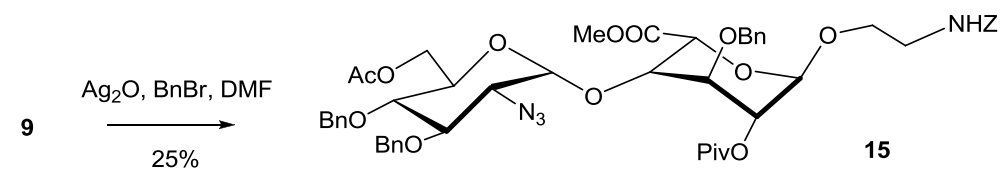

Scheme 4. Benzylation of disaccharide 9.

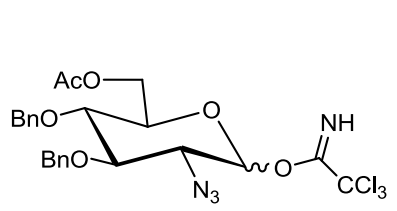

16

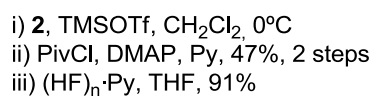

ii) $\mathrm{PivCl}$, DMAP, Py, $47 \%, 2$ steps

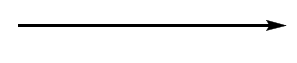

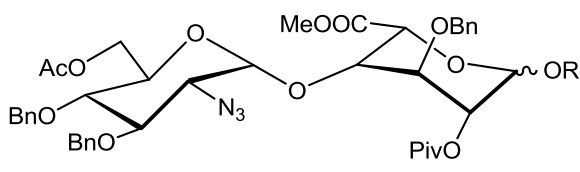

$\mathrm{Cl}_{3} \mathrm{CCN}, \mathrm{K}_{2} \mathrm{CO}_{3}, \mathrm{CH}_{2} \mathrm{Cl}_{2}, 94 \%$

C 17: $\mathrm{R}=\mathrm{H}$

18: $\mathrm{R}=\mathrm{C}(\mathrm{NH}) \mathrm{CCl}_{3}$

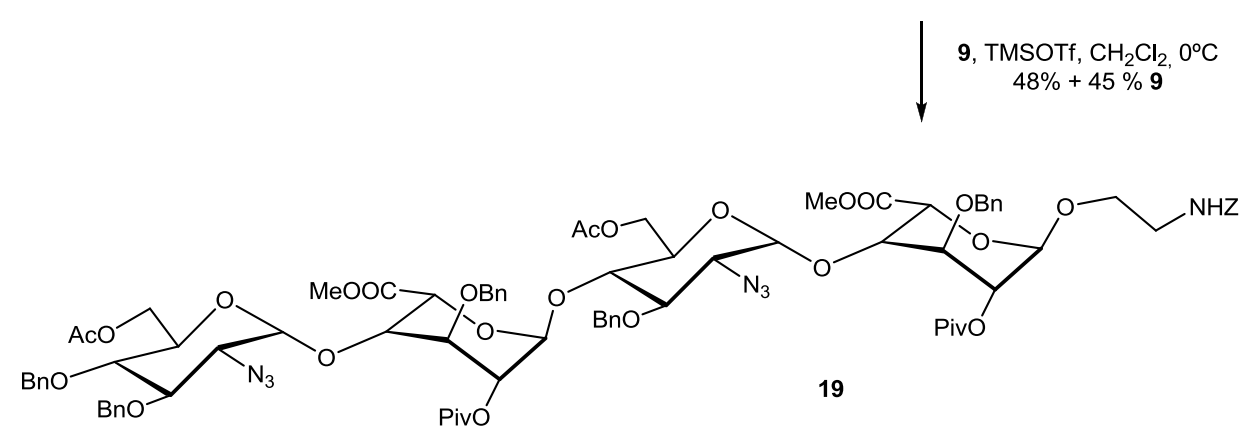

Scheme 5. Synthesis of tetrasaccharide derivative $\mathbf{1 9}$. 
Table 2. Reaction conditions for the glycosylation between 18 and 12

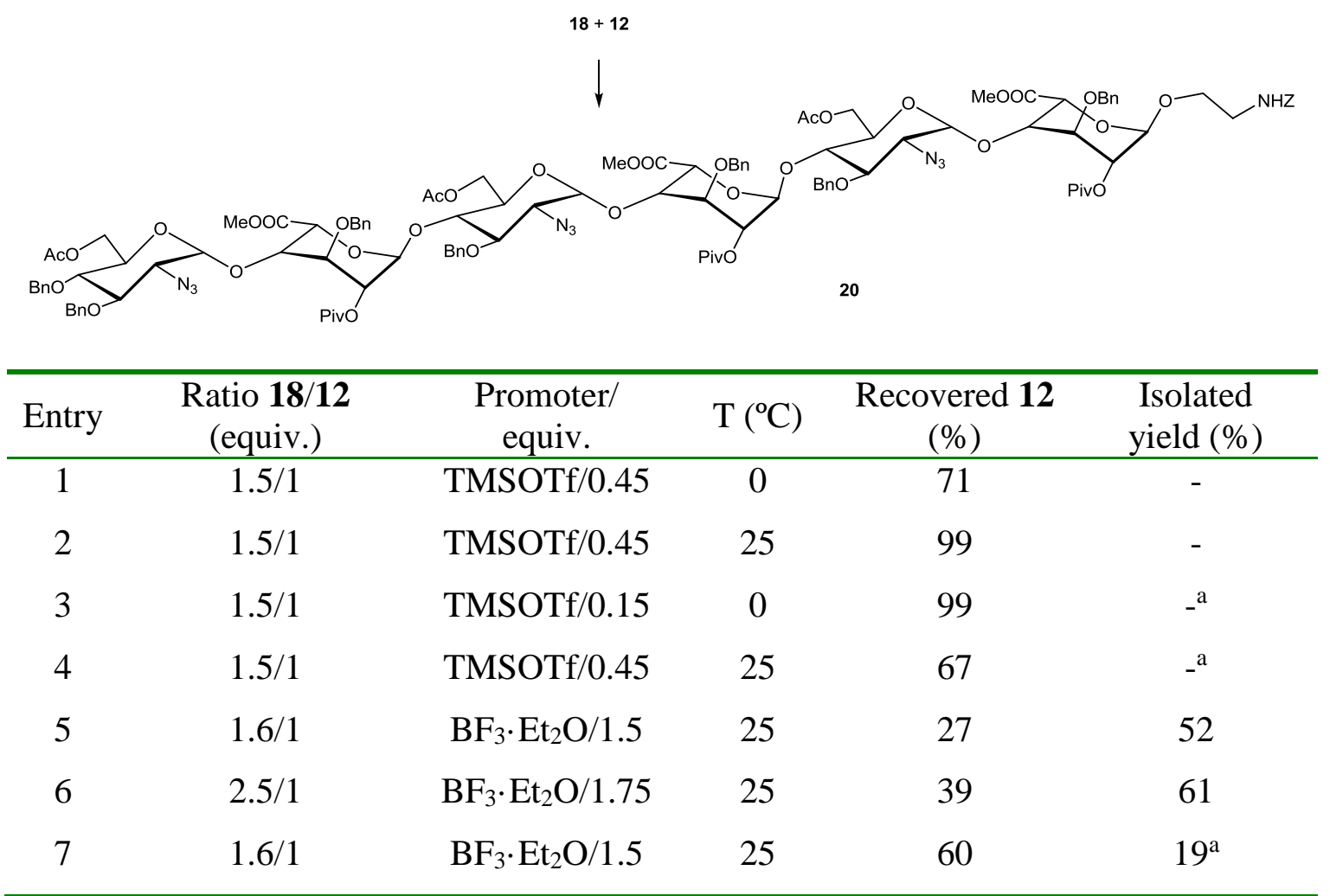

${ }^{\mathrm{a}}$ Inverse procedure

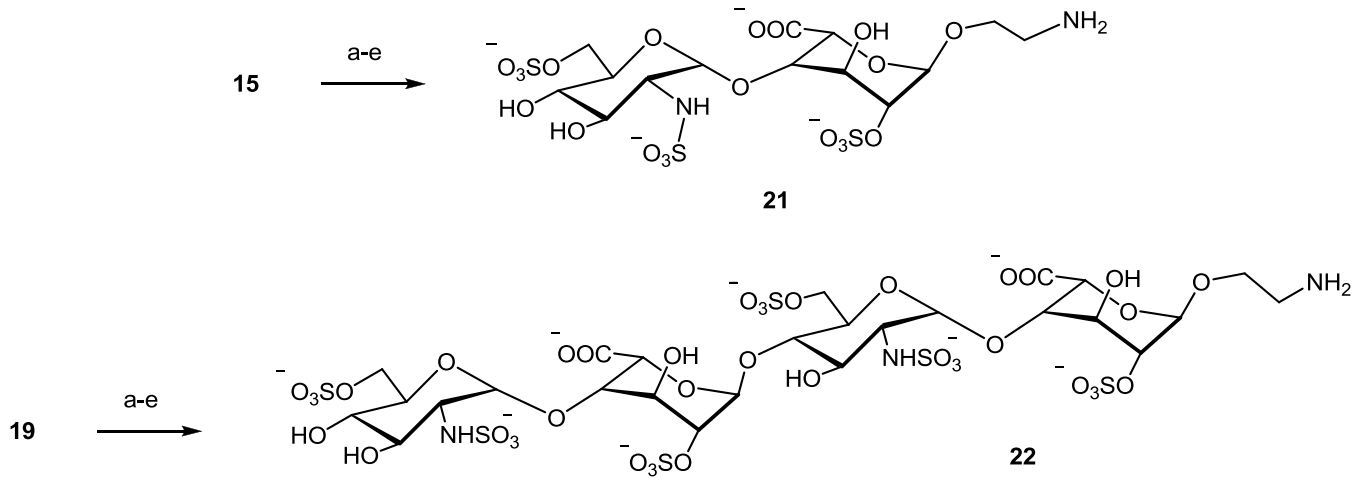

Scheme 6. Synthesis of disaccharide 21 and tetrasaccharide 22. Reaction conditions: a)

$\mathrm{LiOH}, \mathrm{H}_{2} \mathrm{O}_{2}$, THF; $\mathrm{NaOH}, \mathrm{MeOH}$; b) $\mathrm{SO}_{3} \cdot \mathrm{Me}_{3} \mathrm{~N}, \mathrm{DMF}, 100^{\circ} \mathrm{C}, \mathrm{MW}$; c) $\mathrm{Me}_{3} \mathrm{P}, \mathrm{THF}$, $\mathrm{NaOH}$; d) $\mathrm{SO}_{3} \cdot \mathrm{Py}, \mathrm{Py}, \mathrm{Et}_{3} \mathrm{~N}$; e) $\mathrm{H}_{2}, \mathrm{Pd}(\mathrm{OH})_{2}, \mathrm{H}_{2} \mathrm{O} / \mathrm{MeOH}, 21,45 \%$; 22, 36\%, five steps. 


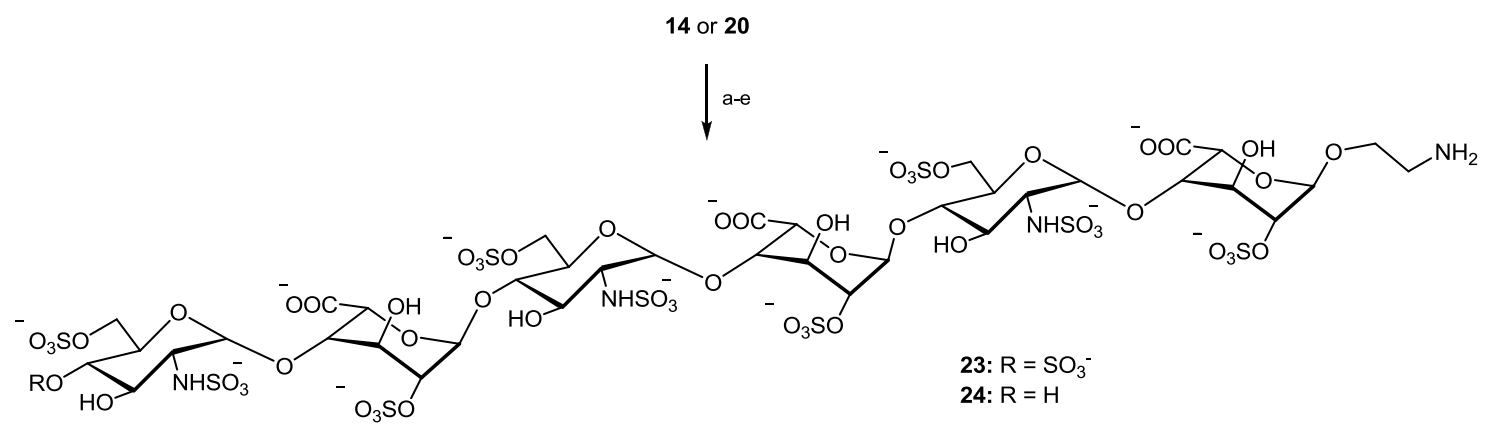

Scheme 7. Synthesis of hexasaccharides $\mathbf{2 3}$ and 24. Reaction conditions: a) LiOH, $\mathrm{H}_{2} \mathrm{O}_{2}$, THF; $\mathrm{NaOH}, \mathrm{MeOH}$; b) $\mathrm{SO}_{3} \cdot \mathrm{Me}_{3} \mathrm{~N}, \mathrm{DMF}, 100^{\circ} \mathrm{C}, \mathrm{MW}$; c) $\mathrm{Me}_{3} \mathrm{P}$, THF, $\mathrm{NaOH}$; d) $\mathrm{SO}_{3} \cdot \mathrm{Me}_{3} \mathrm{~N}, \mathrm{DMF}, \mathrm{Et}_{3} \mathrm{~N}, 60^{\circ} \mathrm{C}, \mathrm{MW}$; e) $\mathrm{H}_{2}, \mathrm{Pd}(\mathrm{OH})_{2}, \mathrm{H}_{2} \mathrm{O} / \mathrm{MeOH}, \mathbf{2 3}, \mathbf{3 0} \%$; 24, $13 \%$, five steps.

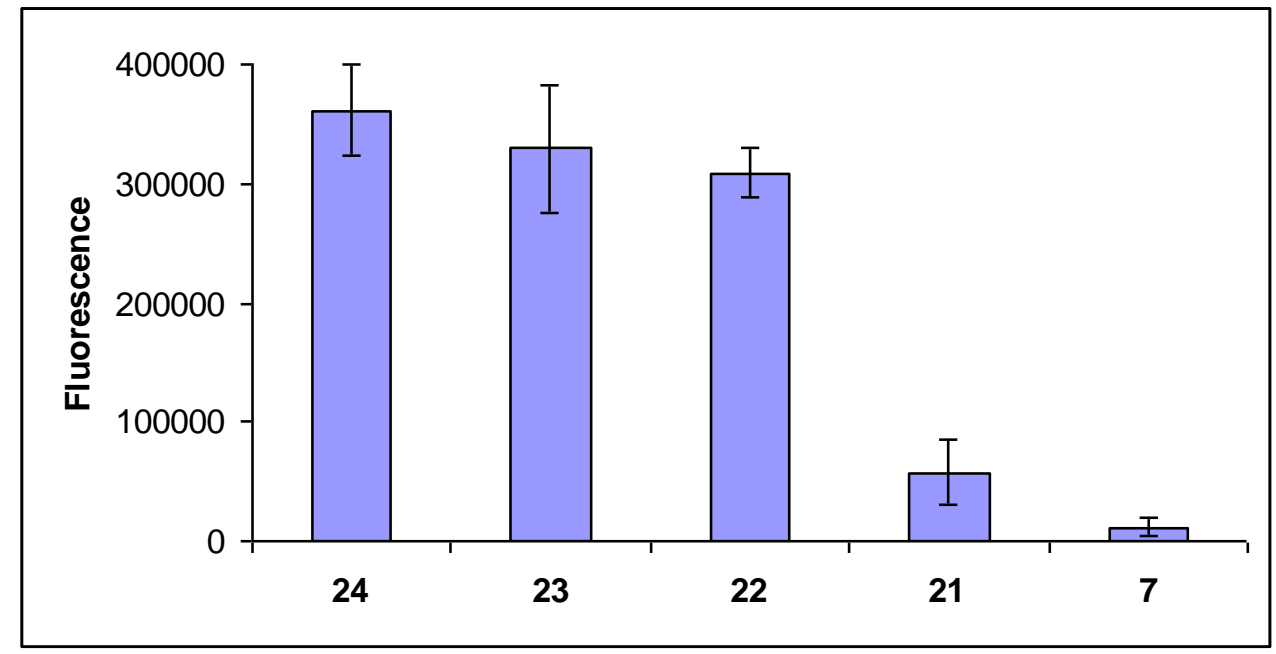

Figure 1. Binding of synthetic heparin-like oligosaccharides to FGF-2. Sugar-coated wells were incubated with $291 \mathrm{nM}$ protein. For each oligosaccharide, fluorescence signals are the average of five replicate wells and the error bars show the standard deviations for these measurements. 

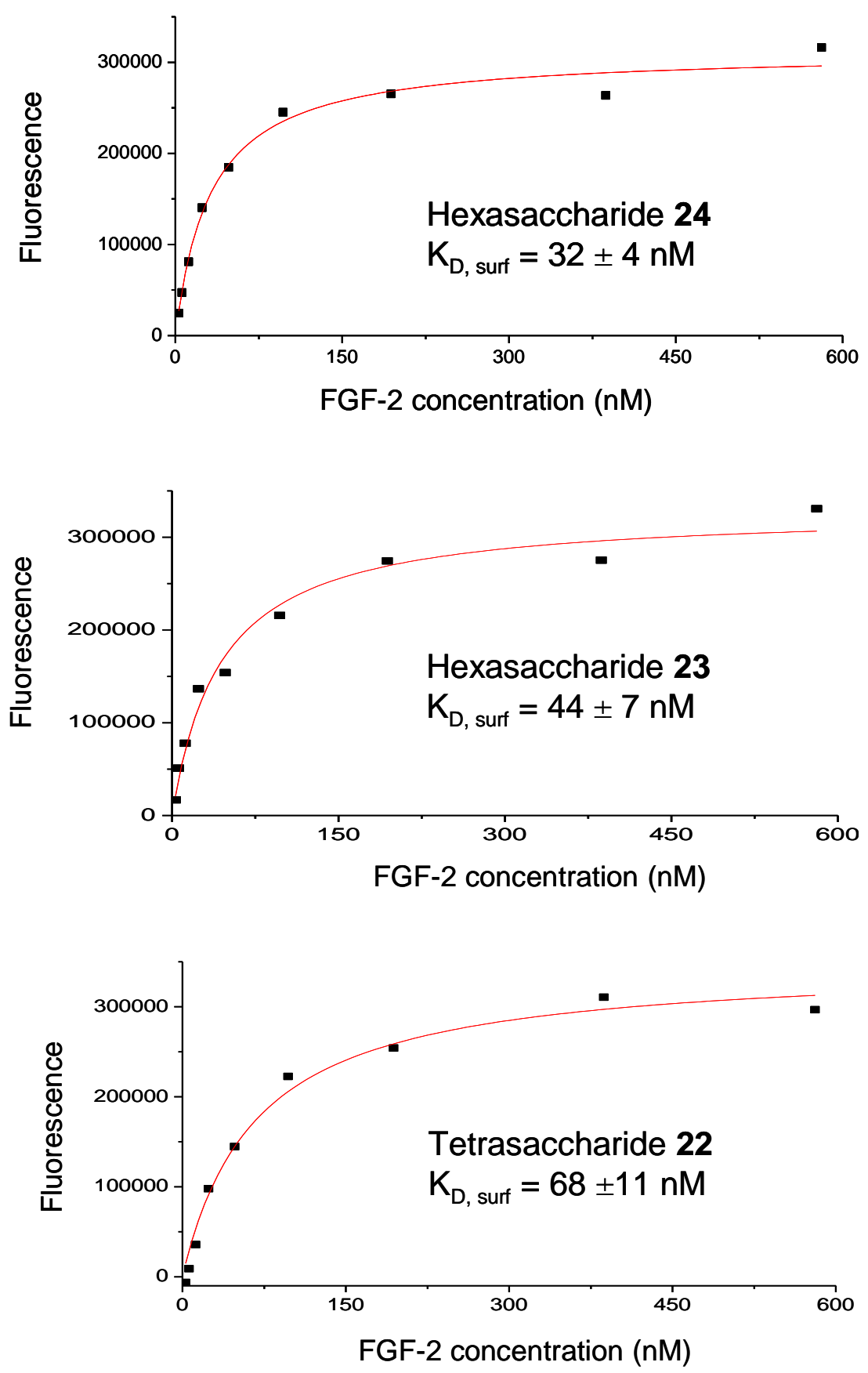

Figure 2. Binding curves for oligosaccharides 22, 23 and 24. Average fluorescence intensities of five replicates were plotted against FGF-2 concentrations. The $\mathrm{K}_{\mathrm{D} \text {, surf }}$ values were obtained by fitting the curves to a Langmuir isotherm for a one-site model of interaction. 


\section{Experimental}

General procedures: Thin layer chromatography (TLC) analyses were performed on silica gel $60 \mathrm{~F}_{254}$ precoated on aluminium plates (Merck) and the compounds were detected by staining with sulfuric acid/ethanol (1:9), with cerium (IV) sulfate (10 g), phosphomolybdic acid (13 g), sulfuric acid $(60 \mathrm{~mL})$ solution in water $(1 \mathrm{~L})$ or with anisaldehyde solution (anisaldehyde $(25 \mathrm{~mL})$ with sulfuric acid $(25 \mathrm{~mL})$, ethanol $(450 \mathrm{~mL})$ and acetic acid $(1 \mathrm{~mL})$ ) followed by heating at over $200^{\circ} \mathrm{C}$. Column chromatography was carried out on silica gel $60(0.2-0.5 \mathrm{~mm}, 0.2-0.063 \mathrm{~mm}$ or $0.040-$ $0.015 \mathrm{~mm}$; Merck). Optical rotations were determined with a Perkin-Elmer 341 polarimeter. ${ }^{1} \mathrm{H}$ - and ${ }^{13} \mathrm{C}-\mathrm{NMR}$ spectra were acquired on Bruker DPX-300, and DRX500 spectrometers. Unit A refers to the reducing end monosaccharide in the NMR data. Electrospray mass spectra (ESI-MS) were carried out with an Esquire 6000 ESI-Ion Trap from Bruker Daltonics. High resolution mass spectra (HR MS) were carried out by the Mass Spectrometry Service, CITIUS, Universidad de Sevilla. ESI-MS of final compounds 21, 22, 23 and 24 were obtained with a QSTAR mass spectrometer (Applied Biosystems) at SIdI, Universidad Autónoma de Madrid. Microwave-based sulfation reactions were performed using a Biotage Initiator Eight synthesizer in sealed reaction vessels.

Binding assays in microtiter plates: Oligosaccharides 21-24 and monosaccharide 7 were dissolved in sodium bicarbonate buffer (50 mM, pH 9.6) containing hexadecyltrimethylammonium bromide $(1 \mathrm{mM})$ to afford $10 \mu \mathrm{M}$ sugar solutions. These solutions were transferred to the wells of Nunc Immobilizer Amino ${ }^{\mathrm{TM}} 384$ microtiter plates (from Thermo Scientific) $(20 \mu \mathrm{L} /$ well). Blank wells were incubated with $100 \mathrm{mM}$ ethanolamine in sodium phosphate buffer ( $50 \mathrm{mM}, \mathrm{pH} 9.6)$ containing hexadecyltrimethylammonium bromide $(1 \mathrm{mM})(80 \mu \mathrm{L} /$ well $)$. All samples were 
performed in replicates of five. The plate was shaken overnight at room temperature and the wells were emptied and washed with water. All wells were then blocked by $1 \mathrm{~h}$ incubation with $100 \mathrm{mM}$ ethanolamine in sodium phosphate buffer and washed with water. $20 \mu \mathrm{L}$ of a recombinant human basic Fibroblast Growth Factor solution (FGF-2, from Peprotech, $5 \mu \mathrm{g} / \mathrm{mL}$ in PBS containing 1\% BSA) were added to each well. The microplate was incubated with gentle agitation at room temperature for $1 \mathrm{~h}$ and washed with PBS containing $1 \%$ Tween 20 and $0.1 \%$ BSA, and water. Rabbit anti-human FGF2 antibody (from Peprotech, $5 \mu \mathrm{g} / \mathrm{mL}$ in PBS containing 1\% BSA, $20 \mu \mathrm{L} /$ well) was added to the wells. The plate was shaken for $1 \mathrm{~h}$ and washed with PBS containing $1 \%$ Tween 20 and $0.1 \%$ BSA, and water. The primary anti-FGF2 antibody was detected by using Alexa Fluor 488 labelled anti-rabbit IgG (from Invitrogen, $20 \mu \mathrm{g} / \mathrm{mL}$ in PBS containing $1 \%$ BSA, $20 \mu \mathrm{L} /$ well). The plate was incubated with shaking in the dark for $1 \mathrm{~h}$ and then washed with PBS containing $1 \%$ Tween 20 and $0.1 \%$ BSA, and water. The fluorescence was read at $535 \mathrm{~nm}$ using a TRIAD multimode microplate reader (from Dynex). The blank well measurements were subtracted from all values.

Determination of surface dissociation constants (KD, surf): Oligosaccharides 22-24 were attached to Nunc Immobilizer Amino ${ }^{\mathrm{TM}} 384$ microplates as described before. The wells were incubated with 9 different concentrations of FGF-2, ranging from 581 to 3 nM. All concentrations and samples were performed in five replicates. The bound protein was detected by using the corresponding primary and secondary antibodies as above. Data were fitted to a Langmuir equation, assuming a one-site model of the interaction.

Methyl 4-O-(6-O-acetyl-2-azido-3-O-benzyl-2-deoxy-4- $O$-levulinoyl- $\alpha$-Dglucopyranosyl)-3- $O$-benzyl-2- $O$-pivaloyl- $\alpha, \beta$-L-idopyranosuronate (3): TMSOTf $(126 \mu \mathrm{L}, 0.70 \mathrm{mmol})$ was added under an $\operatorname{argon}$ atmosphere at $-30^{\circ} \mathrm{C}$ to a mixture of $\mathbf{1}$ 
(10.1 g, $17.4 \mathrm{mmol})$ and 2 (15.2 g, $34.5 \mathrm{mmol})$ in dry $\mathrm{CH}_{2} \mathrm{Cl}_{2}(625 \mathrm{~mL})$. After stirring for $30 \mathrm{~min}$ at $-30^{\circ} \mathrm{C}$, the reaction mixture was neutralized with $\mathrm{Et}_{3} \mathrm{~N}$ and concentrated to dryness. The residue was separated by flash column chromatography (hexane/EtOAc 2:1) to obtain unreacted acceptor $(7.2 \mathrm{~g}, 47 \%)$ and fractions containing the desired disaccharide; these were combined, concentrated, and dissolved in Py (100 mL). Pivaloyl chloride (40 mL) and DMAP (cat.) were added and the solution was stirred at room temperature. After $36 \mathrm{~h}$, the mixture was diluted with $\mathrm{CH}_{2} \mathrm{Cl}_{2}$, washed with $1 \mathrm{M}$ $\mathrm{HCl}$ aqueous solution, saturated $\mathrm{NaHCO}_{3}$ aqueous solution and $\mathrm{H}_{2} \mathrm{O}$, dried $\left(\mathrm{MgSO}_{4}\right)$, filtered, and concentrated in vacuo. The residue was purified by flash column chromatography (hexane/EtOAc $8: 1 \rightarrow 3: 1$ ) to yield the corresponding 2-O-pivaloylated disaccharide (7.6 g, 46\%; 2 steps).

An excess of $(\mathrm{HF})_{n} \cdot \mathrm{Py}(7.6 \mathrm{~mL})$ was added at $-15^{\circ} \mathrm{C}$ to a solution of this disaccharide $(1.52 \mathrm{~g}, 1.61 \mathrm{mmol})$ in dry THF $(40 \mathrm{~mL})$. The reaction was warmed to room temperature and stirred for $28 \mathrm{~h}$ under an argon atmosphere. The mixture was diluted with $\mathrm{CH}_{2} \mathrm{Cl}_{2}$ and washed with $\mathrm{H}_{2} \mathrm{O}$ and saturated $\mathrm{NaHCO}_{3}$ solution until neutral $\mathrm{pH}$. The organic layers were dried $\left(\mathrm{MgSO}_{4}\right)$, filtered and concentrated in vacuo. The residue was purified by column chromatography (hexane-EtOAc 1:1) to afford 3 (880 $\mathrm{mg}, 68 \%$ ) as a mixture of $\alpha / \beta$ anomers. TLC (hexane-EtOAc $1: 1$ ) $\mathrm{R}_{\mathrm{f}} 0.18 ;{ }^{1} \mathrm{H}-\mathrm{NMR}$ (300 MHz, $\mathrm{CDCl}_{3}$ ) (data for major anomer): $\delta 7.38-7.28(\mathrm{~m}, 10 \mathrm{H}, \mathrm{Ar}), 5.36\left(\mathrm{dd}, 1 \mathrm{H}, J_{1,2}\right.$ $\left.=4.4 \mathrm{~Hz}, J_{1, \mathrm{OH}}=8.6 \mathrm{~Hz}, \mathrm{H}-1 \mathrm{~A}\right), 5.06(\mathrm{~m}, 1 \mathrm{H}, \mathrm{H}-4 \mathrm{~B}), 5.04\left(\mathrm{~d}, 1 \mathrm{H}, J_{1,2}=3.5 \mathrm{~Hz}, \mathrm{H}-1 \mathrm{~B}\right)$, 4.88 (m, 1H, H-2A), 4.80 (m, 1H, H-5A), 4.80-4.62 (m, 4H, $\left.\mathrm{CH}_{2}(\mathrm{OBn})\right), 4.19$ (dd, 1H, $\left.J_{5,6 \mathrm{a}}=4.5 \mathrm{~Hz}, J_{6 \mathrm{a}, 6 \mathrm{~b}}=12.5 \mathrm{~Hz}, \mathrm{H}-6 \mathrm{aB}\right), 4.10(\mathrm{~m}, 2 \mathrm{H}, \mathrm{H}-3 \mathrm{~A}, \mathrm{H}-4 \mathrm{~A}), 4.09\left(\mathrm{dd}, 1 \mathrm{H}, J_{5,6 \mathrm{~b}}=\right.$ $2.2 \mathrm{~Hz}, \mathrm{H}-6 \mathrm{bB}), 4.00\left(\mathrm{ddd}, 1 \mathrm{H}, J_{4,5}=10.3 \mathrm{~Hz}, \mathrm{H}-5 \mathrm{~B}\right), 3.86\left(\mathrm{t}, 1 \mathrm{H}, J_{2,3}=J_{3,4}=10.2 \mathrm{~Hz}\right.$, H-3B), 3.80 (s, 3H, COOMe), 3.52 (d, 1H, OH), 3.37 (dd, 1H, H-2B), 2.70 (t, 2H, $\left.\mathrm{CH}_{2}(\mathrm{Lev})\right), 2.56-2.37$ (m, 2H, $\left.\mathrm{CH}_{2}(\mathrm{Lev})\right), 2.16$ (s, 3H, $\left.\mathrm{CH}_{3}(\mathrm{Lev})\right), 2.06$ (s, 3H, 
$\mathrm{CH}_{3}(\mathrm{Ac})$ ), 1.25 (s, 9H, $\left.\left(\mathrm{CH}_{3}\right)_{3}(\mathrm{Piv})\right) ;{ }^{13} \mathrm{C}-\mathrm{NMR}\left(75 \mathrm{MHz}, \mathrm{CDCl}_{3}\right.$ ) (data for major anomer): $\delta 206.2$ (CO(Lev)), 178.5, 171.6, 170.8, 169.6 (CO(Lev, Ac, Piv, COOMe)), 137.5, 137.1 (Ar-C), 128.7-128.1 (Ar-CH), 99.0 (C-1B), 93.7 (C-1A), 77.4 (C-3B), 75.6 (C-4A or C-3A), 74.8, $74.7\left(\mathrm{CH}_{2}(\mathrm{OBn})\right), 74.1$ (C-4A or $\left.\mathrm{C}-3 \mathrm{~A}\right), 71.3(\mathrm{C}-2 \mathrm{~A}), 70.2$ (C-4B), 69.8 (C-5A), 69.2 (C-5B), 63.0 (C-2B), 61.8 (C-6B), 52.5 (COOMe), 39.1 (C(Piv)), $37.9\left(\mathrm{CH}_{2}(\mathrm{Lev})\right), 29.9\left(\mathrm{CH}_{3}(\mathrm{Lev})\right), 28.0\left(\mathrm{CH}_{2}(\mathrm{Lev})\right), 27.3\left(\left(\mathrm{CH}_{3}\right)_{3}(\mathrm{Piv})\right), 20.9$ $\left(\mathrm{CH}_{3}(\mathrm{Ac})\right.$ ); ${ }^{1} \mathrm{H}-\mathrm{NMR}\left(300 \mathrm{MHz}, \mathrm{CDCl}_{3}\right.$ ) (selected data for minor anomer): $\delta 7.38-7.28$ $(\mathrm{m}, 10 \mathrm{H}, \mathrm{Ar}), 5.22\left(\mathrm{dd}, 1 \mathrm{H}, J_{1,2}=2.4 \mathrm{~Hz}, J_{1, \mathrm{OH}}=9.9 \mathrm{~Hz}, \mathrm{H}-1 \mathrm{~A}\right), 4.97\left(\mathrm{~d}, 1 \mathrm{H}, J_{1,2}=3.8\right.$ Hz, H-1B), 4.94 (dd, 1H, J J,3 = 4.9 Hz, H-2A), 4.80-4.62 (m, 4H, $\mathrm{CH}_{2}(\mathrm{OBn})$ ), 4.58 (d, $\left.1 \mathrm{H}, J_{4,5}=3.3 \mathrm{~Hz}, \mathrm{H}-5 \mathrm{~A}\right), 3.80(\mathrm{~m}, 4 \mathrm{H}, \mathrm{COOMe}, \mathrm{OH}), 3.40\left(\mathrm{dd}, 1 \mathrm{H}, J_{2,3}=10.0 \mathrm{~Hz}\right.$, $\mathrm{H}-2 \mathrm{~B}$ ), 1.29 (s, 9H, $\left(\mathrm{CH}_{3}\right)_{3}(\mathrm{Piv})$ ); ${ }^{13} \mathrm{C}-\mathrm{NMR}\left(75 \mathrm{MHz}, \mathrm{CDCl}_{3}\right.$ ) (selected data for minor anomer): $\delta$ 128.7-127.8 (Ar-CH), 98.6 (C-1B), 92.7 (C-1A), 75.0-74.7 $\left(\mathrm{CH}_{2}(\mathrm{OBn})\right)$, 73.6 (C-5A), 70.3 (C-2A), 52.8 (COOMe), 39.2 (C(Piv)), $27.4\left(\left(\mathrm{CH}_{3}\right)_{3}(\mathrm{Piv})\right), 20.9$ $\left(\mathrm{CH}_{3}(\mathrm{Ac})\right)$; HR MS: $m / z$ : calcd for $\mathrm{C}_{39} \mathrm{H}_{49} \mathrm{~N}_{3} \mathrm{O}_{15} \mathrm{Na}$ : 822.3061; found: 822.3064 $[M+\mathrm{Na}]^{+}$.

$O$-(Methyl 4-O-(6-O-acetyl-2-azido-3-O-benzyl-2-deoxy-4-O-levulinoyl- $\alpha$-Dglucopyranosyl)-3- $O$-benzyl-2- $O$-pivaloyl- $\alpha, \beta$-L-idopyranosyluronate)

trichloroacetimidate (4): $\mathrm{K}_{2} \mathrm{CO}_{3}(83 \mathrm{mg}, 0.61 \mathrm{mmol})$ and trichloroacetonitrile $(0.82$ $\mathrm{mL}, 8.25 \mathrm{mmol}$ ) were added at room temperature to a solution of $\mathbf{3}(440 \mathrm{mg}, 0.55$ mmol) in dry $\mathrm{CH}_{2} \mathrm{Cl}_{2}(5 \mathrm{~mL})$. After stirring for $6 \mathrm{~h}$ at room temperature, the reaction mixture was filtered through a pad of Celite and concentrated to dryness. The residue was purified by column chromatography (hexane-EtOAc 2:1 $\left.+1 \% \mathrm{Et}_{3} \mathrm{~N}\right)$ to yield $4(460$ $\mathrm{mg}, 88 \%$ ). TLC (hexane-EtOAc $1: 1) \mathrm{R}_{\mathrm{f}}$ 0.44, 0.51 ( $\alpha$ and $\beta$ anomers); ${ }^{1} \mathrm{H}-\mathrm{NMR}$ (300 $\mathrm{MHz}, \mathrm{CDCl}_{3}$ ) (data for major anomer): $\delta 8.72(\mathrm{~s}, 1 \mathrm{H}, \mathrm{NH}), 7.36-7.25(\mathrm{~m}, 10 \mathrm{H}, \mathrm{Ar}), 6.42$ $\left(\mathrm{d}, 1 \mathrm{H}, J_{1,2}=2.1 \mathrm{~Hz}, \mathrm{H}-1 \mathrm{~A}\right), 5.20$ (bt, $\left.1 \mathrm{H}, \mathrm{H}-2 \mathrm{~A}\right), 5.10$ (bt, $1 \mathrm{H}, J_{3,4}=J_{4,5}=9.6 \mathrm{~Hz}$, 
H-4B), $5.04\left(\mathrm{~d}, 1 \mathrm{H}, J_{1,2}=3.5 \mathrm{~Hz}, \mathrm{H}-1 \mathrm{~B}\right), 4.92\left(\mathrm{~d}, 1 \mathrm{H}, J_{4,5}=3.0 \mathrm{~Hz}, \mathrm{H}-5 \mathrm{~A}\right), 4.86-4.63$ (m, 4H, $\left.\mathrm{CH}_{2}(\mathrm{OBn})\right), 4.25-4.03$ (m, 4H, H-4A, H-6aB, H-6bB, H-3A), 4.01 (m, 1H, H-5B), $3.90\left(\mathrm{t}, 1 \mathrm{H}, J_{2,3}=J_{3,4}=9.2 \mathrm{~Hz}, \mathrm{H}-3 \mathrm{~B}\right), 3.80(\mathrm{~s}, 3 \mathrm{H}, \mathrm{COOMe}), 3.40(\mathrm{dd}, 1 \mathrm{H}$, H-2B), 2.70 (m, 2H, CH $2(\mathrm{Lev})$ ), 2.56-2.37 (m, 2H, $\left.\mathrm{CH}_{2}(\mathrm{Lev})\right), 2.17$ (s, 3H, $\mathrm{CH}_{3}(\mathrm{Lev})$ ), 2.04 (s, 3H, $\left.\mathrm{CH}_{3}(\mathrm{Ac})\right), 1.28\left(\mathrm{~s}, 9 \mathrm{H},\left(\mathrm{CH}_{3}\right)_{3}(\mathrm{Piv})\right) ;{ }^{13} \mathrm{C}-\mathrm{NMR}\left(75 \mathrm{MHz}, \mathrm{CDCl}_{3}\right)$ (data for major anomer): $\delta 206.2(\mathrm{CO}(\mathrm{Lev})), 177.6,171.6,170.8,168.8,(\mathrm{CO}(\mathrm{Lev}, \mathrm{Ac}, \mathrm{Piv}$, COOMe)), $160.3(\mathrm{C}=\mathrm{NH}), 137.5,137.2(\mathrm{Ar}-\mathrm{C}), 128.6-127.7$ (Ar-CH), 98.6 (C-1B), 95.6 (C-1A), $90.8\left(\mathrm{CCl}_{3}\right), 77.5(\mathrm{C}-3 \mathrm{~B}), 74.7,73.3\left(\mathrm{CH}_{2}(\mathrm{OBn})\right), 74.2(\mathrm{C}-4 \mathrm{~A}), 74.1$ (C-3A), 70.0 (C-4B), 69.8 (C-5A), 69.2 (C-5B), 67.2 (C-2A), 63.0 (C-2B), 61.5 (C-6B), 52.5 (COOMe), 39.0 (C(Piv)), 37.9( $\left.\mathrm{CH}_{2}(\mathrm{Lev})\right), 29.9\left(\mathrm{CH}_{3}(\mathrm{Lev})\right), 27.9\left(\mathrm{CH}_{2}(\mathrm{Lev})\right), 27.3$ (( $\left.\mathrm{CH}_{3}\right)_{3}(\mathrm{Piv})$ ), $20.7\left(\mathrm{CH}_{3}(\mathrm{Ac})\right)$; ${ }^{1} \mathrm{H}-\mathrm{NMR}\left(300 \mathrm{MHz}, \mathrm{CDCl}_{3}\right)$ (selected data for minor anomer): $\delta 8.66(\mathrm{~s}, 1 \mathrm{H}, \mathrm{NH}), 7.36-7.25(\mathrm{~m}, 10 \mathrm{H}, \operatorname{Ar}), 6.35\left(\mathrm{~d}, 1 \mathrm{H}, J_{1,2}=2.7 \mathrm{~Hz}\right.$, H-1A), $5.24\left(\mathrm{dd}, 1 \mathrm{H}, J_{2,3}=5.6 \mathrm{~Hz}, \mathrm{H}-2 \mathrm{~A}\right), 5.00\left(\mathrm{~d}, 1 \mathrm{H}, J_{1,2}=3.7 \mathrm{~Hz}, \mathrm{H}-1 \mathrm{~B}\right), 4.86-4.63$ (m, 5H, H-5A, $\mathrm{CH}_{2}(\mathrm{OBn})$ ), 4.33 (t, 1H, H-3A), 3.79 (s, 3H, COOMe), 3.44 (dd, 1H, H2B), 2.70 (m, 2H, $\mathrm{CH}_{2}(\mathrm{Lev})$ ), 2.56-2.37 (m, 2H, $\mathrm{CH}_{2}(\mathrm{Lev})$ ), 2.15 (s, 3H, $\mathrm{CH}_{3}(\mathrm{Lev})$ ), 2.05 (s, 3H, $\left.\mathrm{CH}_{3}(\mathrm{Ac})\right), 1.25$ (s, 9H, $\left.\left(\mathrm{CH}_{3}\right)_{3}(\mathrm{Piv})\right) ;{ }^{13} \mathrm{C}-\mathrm{NMR}\left(75 \mathrm{MHz}, \mathrm{CDCl}_{3}\right)$ (selected data for minor anomer): $\delta 99.5(\mathrm{C}-1 \mathrm{~B}), 94.8(\mathrm{C}-1 \mathrm{~A}), 74.6(\mathrm{C}-3 \mathrm{~A}), 68.1(\mathrm{C}-2 \mathrm{~A}), 63.3(\mathrm{C}-$ 2B), 52.3 (COOMe), 39.1 (C(Piv)), $37.9 \quad\left(\mathrm{CH}_{2}(\mathrm{Lev})\right), \quad 29.9 \quad\left(\mathrm{CH}_{3}(\mathrm{Lev})\right), \quad 27.9$ $\left(\mathrm{CH}_{2}(\mathrm{Lev})\right), \quad 27.3 \quad\left(\left(\mathrm{CH}_{3}\right)_{3}(\mathrm{Piv})\right), \quad 20.7 \quad\left(\mathrm{CH}_{3}(\mathrm{Ac})\right) ; \quad$ ESI $\mathrm{MS}: \quad m / z:$ calcd for $\mathrm{C}_{41} \mathrm{H}_{49} \mathrm{Cl}_{3} \mathrm{~N}_{4} \mathrm{O}_{15} \mathrm{Na}$ : 965.2; found: $965.7[M+\mathrm{Na}]^{+}$.

(4-O-(6-O-acetyl-2-azido-3-O-benzyl-2-deoxy-4- $O$-levulinoyl- $\alpha$-D-glucopyranosyl)3- $O$-benzyl-2- $O$-pivaloyl- $\beta$-L-idopyranosyl)-6,1-lactone (5): TLC (hexane-EtOAc 1:1) $\mathrm{R}_{\mathrm{f}} 0.57 ;{ }^{1} \mathrm{H}-\mathrm{NMR}\left(300 \mathrm{MHz}, \mathrm{CDCl}_{3}\right): \delta 7.37-7.24(\mathrm{~m}, 10 \mathrm{H}, \mathrm{Ar}), 5.86\left(\mathrm{~d}, 1 \mathrm{H}, J_{1,2}=\right.$ $2.1 \mathrm{~Hz}, \mathrm{H}-1 \mathrm{~A}), 5.14\left(\mathrm{~d}, 1 \mathrm{H}, J_{1,2}=3.9 \mathrm{~Hz}, \mathrm{H}-1 \mathrm{~B}\right), 5.09\left(\mathrm{dd}, 1 \mathrm{H}, J_{3,4}=9.3 \mathrm{~Hz}, J_{4,5}=10.2\right.$ $\mathrm{Hz}, \mathrm{H}-4 \mathrm{~B}), 5.01\left(\mathrm{~d}, 1 \mathrm{H}, \mathrm{CH}_{2}(\mathrm{OBn})\right), 4.96\left(\mathrm{dt}, 1 \mathrm{H}, J_{2,3}=6.0 \mathrm{~Hz}, J_{2,4}=1.8 \mathrm{~Hz}, \mathrm{H}-2 \mathrm{~A}\right)$, 
$4.76\left(\mathrm{~m}, 2 \mathrm{H}, \mathrm{CH}_{2}(\mathrm{OBn})\right), 4.73(\mathrm{~m}, 2 \mathrm{H}, \mathrm{H}-4 \mathrm{~A}, \mathrm{H}-5 \mathrm{~A}), 4.66\left(\mathrm{~d}, 1 \mathrm{H}, \mathrm{CH}_{2}(\mathrm{OBn})\right), 4.40$ $\left(\mathrm{ddd}, 1 \mathrm{H}, J_{5,6 \mathrm{a}}=5.1 \mathrm{~Hz}, J_{5,6 \mathrm{~b}}=2.1 \mathrm{~Hz}, \mathrm{H}-5 \mathrm{~B}\right), 4.23\left(\mathrm{dd}, 1 \mathrm{H}, J_{6 \mathrm{a}, 6 \mathrm{~b}}=12.3 \mathrm{~Hz}, \mathrm{H}-6 \mathrm{aB}\right)$, 4.13 (dd, 1H, H-6bB), 3.95 (m, 2H, H-3A, H-3B), 3.60 (dd, 1H, J2,3 = $10.2 \mathrm{~Hz}, \mathrm{H}-2 \mathrm{~B})$, $2.71\left(\mathrm{t}, 2 \mathrm{H}, \mathrm{CH}_{2}(\mathrm{Lev})\right), 2.50$ (m, 2H, $\left.\mathrm{CH}_{2}(\mathrm{Lev})\right), 2.17$ (s, 3H, $\left.\mathrm{CH}_{3}(\mathrm{Lev})\right), 2.05$ (s, 3H, $\left.\mathrm{CH}_{3}(\mathrm{Ac})\right), 1.24\left(\mathrm{~s}, 9 \mathrm{H},\left(\mathrm{CH}_{3}\right)_{3}(\mathrm{Piv})\right) ;{ }^{13} \mathrm{C}-\mathrm{NMR}\left(75 \mathrm{MHz}, \mathrm{CDCl}_{3}\right): \delta 206.2(\mathrm{CO}(\mathrm{Lev}))$, 177.8, 171.7, 170.5, 169.4 (CO(Ac, Lev, Piv, lactone)), 137.6, 137.5, 128.6, 128.2, 128.1, 127.8 (Ar), 100.6 (C-1B), 100.3 (C-1A), 79.4 (C-3A), 77.9 (C-3B), 75.9, 75.4 $\left(\mathrm{CH}_{2}(\mathrm{OBn})\right), 73.5$ (C-2A), 70.9 (C-4A, C-5A), 70.5 (C-4B), 69.1 (C-5B), 63.5 (C-2B), 62.4 (C-6B), 39.0 (C(Piv)), $37.9\left(\mathrm{CH}_{2}(\mathrm{Lev})\right), 29.9\left(\mathrm{CH}_{3}(\mathrm{Lev})\right), 28.0\left(\mathrm{CH}_{2}(\mathrm{Lev})\right), 27.1$ (( $\left.\left.\mathrm{CH}_{3}\right)_{3}(\mathrm{Piv})\right), 20.9\left(\mathrm{CH}_{3}(\mathrm{Ac})\right)$; ESI MS: $\mathrm{m} / z$ : calcd for $\mathrm{C}_{38} \mathrm{H}_{45} \mathrm{~N}_{3} \mathrm{O}_{14} \mathrm{Na}$ : 790.3; found: $790.2[M+\mathrm{Na}]^{+}$.

Methyl ( $N$-benzyloxycarbonyl-2-aminoethyl 4-O-(6-O-acetyl-2-azido-3- $O$-benzyl-2deoxy-4-O-levulinoyl- $\alpha$-D-glucopyranosyl)-3-O-benzyl-2- $O$-pivaloyl- $\alpha-\mathrm{L}-$ idopyranoside) uronate (8): TMSOTf $\left(170 \mu \mathrm{L}\right.$ of a $0.092 \mathrm{M}$ solution in dry $\left.\mathrm{CH}_{2} \mathrm{Cl}_{2}\right)$ was added under an argon atmosphere at $0^{\circ} \mathrm{C}$ to a mixture of $4(280 \mathrm{mg}, 0.30 \mathrm{mmol})$ and benzyl $\mathrm{N}$-(2-hydroxyethyl)-carbamate $(116 \mathrm{mg}, 0.593 \mathrm{mmol})$ in dry $\mathrm{CH}_{2} \mathrm{Cl}_{2}(1.5 \mathrm{~mL})$. After stirring for $15 \mathrm{~min}$ at $0^{\circ} \mathrm{C}$, the reaction mixture was neutralized with $\mathrm{Et}_{3} \mathrm{~N}$ and concentrated to dryness. The residue was purified by column chromatography (tolueneEtOAc 3:2) to afford 8 (224 mg, 77\%). TLC (toluene-EtOAc 3:2) $\mathrm{R}_{\mathrm{f}}$ 0.3; ${ }^{1} \mathrm{H}-\mathrm{NMR}(300$ $\left.\mathrm{MHz}, \mathrm{CDCl}_{3}\right): \delta 7.36-7.28(\mathrm{~m}, 15 \mathrm{H}, \mathrm{Ar}), 5.17(\mathrm{bt}, 1 \mathrm{H}, \mathrm{NH}), 5.08(\mathrm{~m}, 5 \mathrm{H}, \mathrm{H}-1 \mathrm{~A}, \mathrm{H}-1 \mathrm{~B}$, $\left.\mathrm{H}-4 \mathrm{~B}, \mathrm{CH}_{2}(\mathrm{Z})\right), 4.95\left(\mathrm{t}, 1 \mathrm{H}, J_{1,2}=J_{2,3}=4.5 \mathrm{~Hz}, \mathrm{H}-2 \mathrm{~A}\right), 4.79-4.70\left(\mathrm{~m}, 4 \mathrm{H}, \mathrm{CH}_{2}(\mathrm{OBn})\right.$, H-5A (4.73)), $4.65\left(\mathrm{~d}, 1 \mathrm{H}, \mathrm{CH}_{2}(\mathrm{OBn})\right), 4.19\left(\mathrm{dd}, 1 \mathrm{H}, J_{5,6 \mathrm{a}}=4.2 \mathrm{~Hz}, J_{6 \mathrm{a}, 6 \mathrm{~b}}=12.6 \mathrm{~Hz}\right.$, $\mathrm{H}-6 \mathrm{aB}), 4.14\left(\mathrm{t}, 1 \mathrm{H}, J_{3,4}=J_{4,5}=4.8 \mathrm{~Hz}, \mathrm{H}-4 \mathrm{~A}\right), 4.08\left(\mathrm{dd}, 1 \mathrm{H}, J_{5,6 \mathrm{~b}}=2.1 \mathrm{~Hz}, \mathrm{H}-6 \mathrm{bB}\right)$, $4.00\left(\mathrm{ddd}, 1 \mathrm{H}, J_{4,5}=10.2 \mathrm{~Hz}, \mathrm{H}-5 \mathrm{~B}\right), 3.96(\mathrm{t}, 1 \mathrm{H}, \mathrm{H}-3 \mathrm{~A}), 3.87\left(\mathrm{t}, 1 \mathrm{H}, J_{2,3}=J_{3,4}=10.2\right.$ $\mathrm{Hz}, \mathrm{H}-3 \mathrm{~B}), 3.78$ (m, 4H, COOMe, $\mathrm{CH}_{2}-\mathrm{O}$ ), 3.63 (m, 1H, $\left.\mathrm{CH}_{2}-\mathrm{O}\right), 3.41$ (m, 2H, $\left.\mathrm{CH}_{2}-\mathrm{N}\right)$, 
$3.35\left(\mathrm{dd}, 1 \mathrm{H}, J_{1,2}=3.6 \mathrm{~Hz}, \mathrm{H}-2 \mathrm{~B}\right), 2.70\left(\mathrm{t}, 2 \mathrm{H}, \mathrm{CH}_{2}(\mathrm{Lev})\right), 2.46\left(\mathrm{~m}, 2 \mathrm{H}, \mathrm{CH}_{2}(\mathrm{Lev})\right)$, 2.16 (s, 3H, $\left.\mathrm{CH}_{3}(\mathrm{Lev})\right), 2.04$ (s, 3H, $\left.\mathrm{CH}_{3}(\mathrm{Ac})\right), 1.22$ (s, 9H, $\left.\left(\mathrm{CH}_{3}\right)_{3}(\mathrm{Piv})\right) ;{ }^{13} \mathrm{C}-\mathrm{NMR}$ (75 $\left.\mathrm{MHz}, \mathrm{CDCl}_{3}\right): \delta 206.2(\mathrm{CO}(\mathrm{Lev})), 177.6,171.6,170.7,169.7$ (CO(Lev, Ac, Piv, COOMe)), 156.5 (CO(Z)), 137.5-136.6 (Ar-C), 128.6-127.7 (Ar-CH), 99.2 (C-1A), 98.3 (C-1B), 77.4 (C-3B), $75.4(\mathrm{C}-3 \mathrm{~A}), 74.8\left(\mathrm{CH}_{2}(\mathrm{OBn})\right), 74.1(\mathrm{C}-4 \mathrm{~A}), 73.3\left(\mathrm{CH}_{2}(\mathrm{OBn})\right)$, $70.2\left(\mathrm{CH}_{2}(\mathrm{Z})\right), 70.0(\mathrm{C}-2 \mathrm{~A}), 69.7$ (C-5A), 69.1 (C-5B), $68.3\left(\mathrm{CH}_{2}-\mathrm{O}\right), 66.9(\mathrm{C}-4 \mathrm{~B}), 62.9$ (C-2B), 61.8 (C-6B), $52.5(\mathrm{COOMe}), 40.9\left(\mathrm{CH}_{2}-\mathrm{N}\right), 38.9(\mathrm{C}(\mathrm{Piv})), 37.9\left(\mathrm{CH}_{2}(\mathrm{Lev})\right)$, $29.8\left(\mathrm{CH}_{3}(\mathrm{Lev})\right), 27.9\left(\mathrm{CH}_{2}(\mathrm{Lev})\right), 27.2\left(\left(\mathrm{CH}_{3}\right)_{3}(\mathrm{Piv})\right), 20.9\left(\mathrm{CH}_{3}(\mathrm{Ac})\right)$; HR MS: $m / z$ : calcd for $\mathrm{C}_{49} \mathrm{H}_{60} \mathrm{~N}_{4} \mathrm{O}_{17} \mathrm{Na}$ : 999.3851; found: $999.3880[M+\mathrm{Na}]^{+}$.

Methyl ( $N$-benzyloxycarbonyl-2-aminoethyl 4-O-(6-O-acetyl-2-azido-3-O-benzyl-2deoxy- $\alpha$-D-glucopyranosyl)-3- $O$-benzyl-2- $O$-pivaloyl- $\alpha$-L-idopyranoside) uronate (9): Compound 8 (250 mg, $0.26 \mathrm{mmol})$ was dissolved in $\mathrm{CH}_{2} \mathrm{Cl}_{2}(1.7 \mathrm{~mL})$ and hydrazine monohydrate $(1.0 \mathrm{~mL}$ of a $0.5 \mathrm{M}$ solution in $\mathrm{Py} / \mathrm{AcOH} 3: 2)$ was added. After stirring at room temperature for $2 \mathrm{~h}$, the reaction mixture was quenched with acetone (1.5 mL). The mixture was diluted with $\mathrm{CH}_{2} \mathrm{Cl}_{2}$ and washed with $\mathrm{H}_{2} \mathrm{O}$. The organic layer was dried $\left(\mathrm{MgSO}_{4}\right)$, filtered and concentrated in vacuo. The residue was purified by column chromatography (hexane-EtOAc 1:1) to yield 9 (204 mg, 91\%). TLC (hexane-EtOAc 1:1) $\mathrm{R}_{\mathrm{f}} 0.5 ;[\alpha]_{\mathrm{D}}^{20}+1^{\mathrm{o}}\left(c\right.$ 1.2, $\left.\mathrm{CH}_{2} \mathrm{Cl}_{2}\right) ;{ }^{1} \mathrm{H}-\mathrm{NMR}\left(300 \mathrm{MHz}, \mathrm{CDCl}_{3}\right): \delta$ 7.42-7.29 (m, $15 \mathrm{H}, \mathrm{Ar}), 5.19$ (bt, 1H, NH), 5.08 (m, 3H, H-1A, $\left.\mathrm{CH}_{2}(\mathrm{Z})\right), 5.03$ (d, 1H, $\left.J_{1,2}=3.3 \mathrm{~Hz}, \mathrm{H}-1 \mathrm{~B}\right), 4.95\left(\mathrm{t}, 1 \mathrm{H}, J_{1,2}=J_{2,3}=4.5 \mathrm{~Hz}, \mathrm{H}-2 \mathrm{~A}\right), 4.85\left(\mathrm{~m}, 2 \mathrm{H}, \mathrm{CH}_{2}(\mathrm{OBn})\right)$, $4.75\left(\mathrm{~m}, 2 \mathrm{H}, \mathrm{CH}_{2}(\mathrm{OBn})\right), 4.72\left(\mathrm{~d}, 1 \mathrm{H}, J_{4,5}=4.2 \mathrm{~Hz}, \mathrm{H}-5 \mathrm{~A}\right), 4.55\left(\mathrm{dd}, 1 \mathrm{H}, J_{5,6 \mathrm{a}}=3.6 \mathrm{~Hz}\right.$, $\left.J_{6 \mathrm{a}, 6 \mathrm{~b}}=12.6 \mathrm{~Hz}, \mathrm{H}-6 \mathrm{aB}\right), 4.17\left(\mathrm{dd}, 1 \mathrm{H}, J_{5,6 \mathrm{~b}}=2.0 \mathrm{~Hz}, \mathrm{H}-6 \mathrm{bB}\right), 4.12(\mathrm{t}, 1 \mathrm{H}, \mathrm{H}-4 \mathrm{~A}), 3.97$ (t, 1H, $\left.J_{3,4}=5.1 \mathrm{~Hz}, \mathrm{H}-3 \mathrm{~A}\right), 3.81\left(\mathrm{~m}, 2 \mathrm{H}, \mathrm{H}-5 \mathrm{~B}, \mathrm{CH}_{2}-\mathrm{O}\right), 3.77$ (s, 3H, COOMe), 3.72 $\left(\mathrm{dd}, 1 \mathrm{H}, J_{2,3}=10.2 \mathrm{~Hz}, J_{3,4}=9.0 \mathrm{~Hz}, \mathrm{H}-3 \mathrm{~B}\right), 3.63\left(\mathrm{~m}, 1 \mathrm{H}, \mathrm{CH}_{2}-\mathrm{O}\right), 3.47(\mathrm{~m}, 1 \mathrm{H}, \mathrm{H}-4 \mathrm{~B})$, $3.41\left(\mathrm{~m}, 2 \mathrm{H}, \mathrm{CH}_{2}-\mathrm{N}\right), 3.23(\mathrm{dd}, 1 \mathrm{H}, \mathrm{H}-2 \mathrm{~B}), 2.09$ (s, 3H, $\left.\mathrm{CH}_{3}(\mathrm{Ac})\right), 1.21(\mathrm{~s}, 9 \mathrm{H}$, 
$\left.\left(\mathrm{CH}_{3}\right)_{3}(\mathrm{Piv})\right) ;{ }^{13} \mathrm{C}-\mathrm{NMR}\left(75 \mathrm{MHz}, \mathrm{CDCl}_{3}\right): \delta 177.7,172.1,169.8$ (CO(Ac, Piv, COOMe)), 156.5 (CO(Z)), 137.9-136.6 (Ar-C), 128.8-127.7 (Ar-CH), 99.1 (C-1A), 98.6 (C-1B), 78.9 (C-3B), $75.6(\mathrm{C}-3 \mathrm{~A}), 75.3\left(\mathrm{CH}_{2}(\mathrm{OBn})\right), 74.0(\mathrm{C}-4 \mathrm{~A}), 73.3\left(\mathrm{CH}_{2}(\mathrm{OBn})\right)$, 71.2 (C-5B), 70.6 (C-4B), 70.1 (C-2A), $70.0(\mathrm{C}-5 \mathrm{~A}), 68.4\left(\mathrm{CH}_{2}-\mathrm{O}\right), 66.8\left(\mathrm{CH}_{2}(\mathrm{Z})\right), 62.8$ (C-2B, C-6B), 52.5 (COOMe), $40.9\left(\mathrm{CH}_{2}-\mathrm{N}\right), 38.9$ (C(Piv)), $27.2\left(\left(\mathrm{CH}_{3}\right)_{3}(\mathrm{Piv})\right), 20.9$ $\left(\mathrm{CH}_{3}(\mathrm{Ac})\right)$; HR MS: $m / z$ : calcd for $\mathrm{C}_{44} \mathrm{H}_{54} \mathrm{~N}_{4} \mathrm{O}_{15} \mathrm{Na}$ : 901.3483 ; found: 901.3514 $[M+\mathrm{Na}]^{+}$.

\section{$N$-Benzyloxycarbonyl-2-aminoethyl (6-O-acetyl-2-azido-3- $O$-benzyl-}

2-deoxy-4-O-levulinoyl- $\alpha$-D-glucopyranosyl)-( $1 \rightarrow 4)-($ methyl

3-O-benzyl-2-Opivaloyl- $\alpha$-L-idopyranosyluronate)-( $1 \rightarrow 4)$-(6-O-acetyl-2-azido-3-O-benzyl-2-deoxy$\alpha$-D-glucopyranosyl)-(1 $\rightarrow 4)$-methyl 3-O-benzyl-2-O-pivaloyl- $\alpha-\mathrm{L}-$ idopyranosiduronate (10): TMSOTf (305 $\mu \mathrm{L}$ of a $0.18 \mathrm{M}$ solution in dry $\mathrm{CH}_{2} \mathrm{Cl}_{2}$ ) was added under an argon atmosphere at $0^{\circ} \mathrm{C}$ to a mixture of $\mathbf{9}(107 \mathrm{mg}, 0.122 \mathrm{mmol})$ and 4 $(172 \mathrm{mg}, 0.183 \mathrm{mmol})$ in dry $\mathrm{CH}_{2} \mathrm{Cl}_{2}(1.2 \mathrm{~mL})$. After stirring for $20 \mathrm{~min}$ at $0^{\circ} \mathrm{C}$, the reaction mixture was neutralized with $\mathrm{Et}_{3} \mathrm{~N}$ and concentrated to dryness. The residue was purified by column chromatography (toluene-EtOAc $4: 1 \rightarrow 2: 1)$ to afford $\mathbf{1 0}$ (150 $\mathrm{mg}, 74 \%)$, unreacted $9(25 \mathrm{mg}, 23 \%)$ and 11 (42 mg, $0.054 \mathrm{mmol})$. TLC (hexaneEtOAc 2:3) $\mathrm{R}_{\mathrm{f}} 0.57 ;[\alpha]_{\mathrm{D}}^{20}+8^{\mathrm{o}}\left(c \quad 0.9, \mathrm{CH}_{2} \mathrm{Cl}_{2}\right) ;{ }^{1} \mathrm{H}-\mathrm{NMR}\left(500 \mathrm{MHz}, \mathrm{CDCl}_{3}\right): \delta$ 7.34-7.25 (m, 25H, Ar), $5.26\left(\mathrm{~d}, 1 \mathrm{H}, J_{1,2}=5.0 \mathrm{~Hz}, \mathrm{H}-1 \mathrm{C}\right), 5.16(\mathrm{bt}, 1 \mathrm{H}, \mathrm{NH}), 5.11(\mathrm{~d}$, $\left.1 \mathrm{H}, J_{1,2}=4.5 \mathrm{~Hz}, \mathrm{H}-1 \mathrm{~A}\right), 5.10-5.06\left(\mathrm{~m}, 3 \mathrm{H}, \mathrm{H}-4 \mathrm{D}, \mathrm{CH}_{2}(\mathrm{Z})\right), 5.05\left(\mathrm{~d}, 1 \mathrm{H}, J_{1,2}=3.6 \mathrm{~Hz}\right.$, H-1D), $5.03\left(\mathrm{~d}, 1 \mathrm{H}, J_{1,2}=3.6 \mathrm{~Hz}, \mathrm{H}-1 \mathrm{~B}\right), 4.98\left(\mathrm{t}, 1 \mathrm{H}, J_{2,3}=5.0 \mathrm{~Hz}, \mathrm{H}-2 \mathrm{C}\right), 4.94-4.92(\mathrm{~m}$, 2H, H-2A, $\left.\mathrm{CH}_{2}(\mathrm{OBn})\right), 4.80-4.63$ (m, 9H, $\mathrm{CH}_{2}(\mathrm{OBn}), \mathrm{H}-5 \mathrm{~A}$ (4.67), H-5C (4.65)), 4.36-4.30 (m, 2H, H-6aB, H-6bB), $4.16\left(\mathrm{dd}, 1 \mathrm{H}, J_{5,6 \mathrm{a}}=4.0 \mathrm{~Hz}, J_{6 \mathrm{a}, 6 \mathrm{~b}}=12.5 \mathrm{~Hz}, \mathrm{H}-6 \mathrm{aD}\right)$, $4.11\left(\mathrm{t}, 1 \mathrm{H}, J_{3,4}=J_{4,5}=5.0 \mathrm{~Hz}, \mathrm{H}-4 \mathrm{~A}\right), 4.07\left(\mathrm{t}, 1 \mathrm{H}, J_{3,4}=J_{4,5}=5.0 \mathrm{~Hz}, \mathrm{H}-4 \mathrm{C}\right), 4.02(\mathrm{dd}$, $\left.1 \mathrm{H}, J_{5,6 \mathrm{~b}}=2.0 \mathrm{~Hz}, \mathrm{H}-6 \mathrm{bD}\right), 3.96-3.93(\mathrm{~m}, 5 \mathrm{H}, \mathrm{H}-3 \mathrm{~A}, \mathrm{H}-3 \mathrm{C}, \mathrm{H}-4 \mathrm{~B}, \mathrm{H}-5 \mathrm{~B}, \mathrm{H}-5 \mathrm{D})$, 
3.84-3.80 (m, 2H, H-3D, $\left.\mathrm{CH}_{2}-\mathrm{O}\right), 3.75-3.70$ (m, 4H, H-3B, COOMe), 3.64-3.59 (m, 4H, COOMe, $\mathrm{CH}_{2}-\mathrm{O}$ ), 3.40 (m, 2H, $\left.\mathrm{CH}_{2}-\mathrm{N}\right), 3.35$ (dd, 1H, J J,3 $\left.=10.3 \mathrm{~Hz}, \mathrm{H}-2 \mathrm{D}\right), 3.29$ (dd, $\left.1 \mathrm{H}, J_{2,3}=10.3 \mathrm{~Hz}, \mathrm{H}-2 \mathrm{~B}\right), 2.74-2.66\left(\mathrm{~m}, 2 \mathrm{H}, \mathrm{CH}_{2}(\mathrm{Lev})\right), 2.52-2.36\left(\mathrm{~m}, 2 \mathrm{H}, \mathrm{CH}_{2}(\mathrm{Lev})\right)$, 2.16 (s, 3H, $\left.\mathrm{CH}_{3}(\mathrm{Lev})\right), 2.11,1.99$ (2s, 6H, $\left.\mathrm{CH}_{3}(\mathrm{Ac})\right)$, 1.22, 1.21 (2s, 18H, $\left.\left(\mathrm{CH}_{3}\right)_{3}(\mathrm{Piv})\right)$; ${ }^{13} \mathrm{C}-\mathrm{NMR}\left(125 \mathrm{MHz}, \mathrm{CDCl}_{3}\right): \delta 206.1$ (CO(Lev)), 177.6, 177.3, 171.5, 170.7, 170.6, 169.8, 169.5 (CO(Lev, Ac, Piv, COOMe)), $156.4(\mathrm{CO}(\mathrm{Z}))$, 137.8-136.6 (Ar-C), 129.1-127.5 (Ar-CH), 99.1 (C-1A), 98.4 (C-1D), 98.1 (C-1B), 98.0 (C-1C), 78.1 (C-3B), 77.4 (C-3D), 76.0 (C-3C), 75.9 (C-3A), 75.0 (C-4B, $\left.\mathrm{CH}_{2}(\mathrm{OBn})\right), 74.7$ $\left(\mathrm{CH}_{2}(\mathrm{OBn})\right), 74.0$ (C-4C), 73.8 (C-4A), $73.7\left(\mathrm{CH}_{2}(\mathrm{OBn})\right), 73.5\left(\mathrm{CH}_{2}(\mathrm{OBn})\right), 70.5$ (C-2C), 70.4 (C-2A, C-5C), 70.3 (C-5A), $70.0\left(\mathrm{CH}_{2}(\mathrm{Z})\right), 69.8$ (C-5B), 69.0 (C-5D), 68.4 ( $\left.\mathrm{CH}_{2}-\mathrm{O}\right), 66.8$ (C-4D), 63.0 (C-2B), 62.9 (C-2D), 62.1 (C-6B), 61.6 (C-6D), 52.4, 52.1 (COOMe), $40.9\left(\mathrm{CH}_{2}-\mathrm{N}\right), 38.9$ (C(Piv)), $37.8\left(\mathrm{CH}_{2}(\mathrm{Lev})\right), 29.8\left(\mathrm{CH}_{3}(\mathrm{Lev})\right), 27.9$ $\left(\mathrm{CH}_{2}(\mathrm{Lev})\right)$, 27.2, $27.1\left(\left(\mathrm{CH}_{3}\right)_{3}(\mathrm{Piv})\right), 20.9,20.8\left(\mathrm{CH}_{3}(\mathrm{Ac})\right)$; HR MS: m/z: calcd for $\mathrm{C}_{83} \mathrm{H}_{101} \mathrm{~N}_{7} \mathrm{O}_{29} \mathrm{Na}$ : 1682.6541 ; found: $1682.6498[M+\mathrm{Na}]^{+}$.

Methyl 4-O-(6-O-acetyl-2-azido-3-O-benzyl-2-deoxy-4- $O$-levulinoyl- $\alpha$-Dglucopyranosyl)-1,5-anhydro-3-O-benzyl-2-O-pivaloyl-L- $x y l o$-hex-1-

enitoluronate (11): TLC (toluene-EtOAc 2:1) $\mathrm{R}_{\mathrm{f}}$ 0.33; $[\alpha]_{\mathrm{D}}^{20}+6^{\circ}\left(c \quad 1.0, \mathrm{CHCl}_{3}\right.$ ); ${ }^{1} \mathrm{H}-\mathrm{NMR}\left(500 \mathrm{MHz}, \mathrm{CDCl}_{3}\right.$ ): $\delta$ 7.38-7.25 (m, 10H, Ar), 6.79 (s, 1H, H-1A), 5.07 (dd, $\left.1 \mathrm{H}, J_{3,4}=J_{4,5}=9.7 \mathrm{~Hz}, \mathrm{H}-4 \mathrm{~B}\right), 4.98\left(\mathrm{~d}, 1 \mathrm{H}, J_{1,2}=3.3 \mathrm{~Hz}, \mathrm{H}-1 \mathrm{~B}\right), 4.79(\mathrm{~d}, 1 \mathrm{H}$, $\left.\mathrm{CH}_{2}(\mathrm{OBn})\right), 4.67$ (bs, 2H, $\left.\mathrm{CH}_{2}(\mathrm{OBn})\right), 4.65$ (d, 1H, $\left.\mathrm{CH}_{2}(\mathrm{OBn})\right), 4.58$ (bs, 1H, H-5A), $4.35(\mathrm{~m}, 1 \mathrm{H}, \mathrm{H}-4 \mathrm{~A}), 4.19\left(\mathrm{dd}, 1 \mathrm{H}, J_{5,6 \mathrm{a}}=4.0 \mathrm{~Hz}, J_{6 \mathrm{a}, 6 \mathrm{~b}}=12.1 \mathrm{~Hz}, \mathrm{H}-6 \mathrm{aB}\right), 4.19$ (bd, $1 \mathrm{H}$, $\left.J_{3,4}=1.9 \mathrm{~Hz}, \mathrm{H}-3 \mathrm{~A}\right), 4.09\left(\mathrm{dd}, 1 \mathrm{H}, J_{5,6 \mathrm{~b}}=2.1 \mathrm{~Hz}, \mathrm{H}-6 \mathrm{bB}\right), 3.96\left(\mathrm{dd}, 1 \mathrm{H}, J_{2,3}=9.8 \mathrm{~Hz}\right.$, H-3B), 3.83 (m, 4H, H-5B, COOMe), 3.35 (dd, 1H, H-2B), 2.72-2.29 (m, 4H, $\left.\mathrm{CH}_{2}(\mathrm{Lev})\right), 2.15$ (s, 3H, $\left.\mathrm{CH}_{3}(\mathrm{Lev})\right), 2.06$ (s, 3H, $\left.\mathrm{CH}_{3}(\mathrm{Ac})\right), 1.24$ (s, 9H, $\left(\mathrm{CH}_{3}\right)_{3}(\mathrm{Piv})$ ); ${ }^{13} \mathrm{C}-\mathrm{NMR}\left(75 \mathrm{MHz}, \mathrm{CDCl}_{3}\right.$ ) (Significant data from HMQC experiment): $\delta 138.6$ 
(C-1A), 97.6 (C-1B), 77.0 (C-3B), $74.5\left(\mathrm{CH}_{2}(\mathrm{OBn})\right), 73.2$ (C-4A), 72.0 (C-5A), 71.9 $\left(\mathrm{CH}_{2}(\mathrm{OBn})\right), 69.6$ (C-4B, C-3A), 69.0 (C-5B), 62.7 (C-2B), 61.4 (C-6B), 52.5 (COOMe), $38.2\left(\mathrm{CH}_{2}(\mathrm{Lev})\right), 29.5\left(\mathrm{CH}_{3}(\mathrm{Lev})\right), 28.4\left(\mathrm{CH}_{2}(\mathrm{Lev})\right), 26.9\left(\left(\mathrm{CH}_{3}\right)_{3}(\mathrm{Piv})\right)$, $20.7\left(\mathrm{CH}_{3}(\mathrm{Ac})\right)$; ESI MS: m/z: calcd for $\mathrm{C}_{39} \mathrm{H}_{47} \mathrm{~N}_{3} \mathrm{O}_{14} \mathrm{Na}$ : 804.3; found: $804.1[M+\mathrm{Na}]^{+}$; HR MS: $m / z$ : calcd for $\mathrm{C}_{39} \mathrm{H}_{47} \mathrm{~N}_{3} \mathrm{O}_{14} \mathrm{Na}$ : 804.2956; found: 804.2982 [M+Na] $]^{+}$

\section{$\mathrm{N}$-Benzyloxycarbonyl-2-aminoethyl (6-O-acetyl-2-azido-3- $O$-benzyl-}

2-deoxy- $\alpha$-D-glucopyranosyl)-(1 $\rightarrow 4)$-(methyl

3-O-benzyl-2-O-pivaloyl- $\alpha$-Lidopyranosyluronate)-(1 $\rightarrow 4)-(6-O$-acetyl-2-azido-3- $O$-benzyl-2-deoxy- $\alpha$-Dglucopyranosyl)-(1 $\rightarrow 4)$-methyl $\quad 3-O$-benzyl-2- $O$-pivaloyl- $\alpha$-L-idopyranosiduronate (12): Compound 10 (110 mg, $0.066 \mathrm{mmol})$ was dissolved in $\mathrm{CH}_{2} \mathrm{Cl}_{2}(1.0 \mathrm{~mL})$ and hydrazine monohydrate $(0.26 \mathrm{~mL}$ of a $0.5 \mathrm{M}$ solution in Py/AcOH 3:2) was added. After stirring at room temperature for $2 \mathrm{~h}$, the reaction mixture was quenched with acetone (0.5 mL). The mixture was diluted with $\mathrm{CH}_{2} \mathrm{Cl}_{2}$ and washed with $\mathrm{H}_{2} \mathrm{O}$. The organic layer was dried $\left(\mathrm{MgSO}_{4}\right)$, filtered and concentrated in vacuo. The residue was purified by column chromatography (toluene-EtOAc 5:2) to yield $\mathbf{1 2}$ (85 mg, 82\%). TLC (hexane-EtOAc 2:3) $\mathrm{R}_{\mathrm{f}} 0.63 ;[\alpha]_{\mathrm{D}}^{20}-2^{\mathrm{o}}\left(c\right.$ 1.0, $\left.\mathrm{CHCl}_{3}\right) ;{ }^{1} \mathrm{H}-\mathrm{NMR}\left(300 \mathrm{MHz}, \mathrm{CDCl}_{3}\right): \delta$ 7.41-7.28 (m, 25H, Ar), $5.29\left(\mathrm{~d}, 1 \mathrm{H}, J_{1,2}=4.6 \mathrm{~Hz}, \mathrm{H}-1 \mathrm{C}\right), 5.14(\mathrm{bt}, 1 \mathrm{H}, \mathrm{NH}), 5.11(\mathrm{~d}$, $\left.1 \mathrm{H}, J_{1,2}=4.7 \mathrm{~Hz}, \mathrm{H}-1 \mathrm{~A}\right), 5.09\left(\mathrm{~m}, 2 \mathrm{H}, \mathrm{CH}_{2}(\mathrm{Z})\right), 5.04-5.00(\mathrm{~m}, 2 \mathrm{H}, \mathrm{H}-1 \mathrm{D}, \mathrm{H}-1 \mathrm{~B})$, 4.99-4.96 (m, 1H, H-2C), 4.95-4.91 (m, 2H, H-2A, $\left.\mathrm{CH}_{2}(\mathrm{OBn})\right), 4.89-4.82(\mathrm{~m}, 2 \mathrm{H}$, $\left.\mathrm{CH}_{2}(\mathrm{OBn})\right), 4.81-4.66\left(\mathrm{~m}, 6 \mathrm{H}, \mathrm{CH}_{2}(\mathrm{OBn}), \mathrm{H}-5 \mathrm{~A}(4.67)\right), 4.60\left(\mathrm{~d}, 1 \mathrm{H}, J_{4,5}=4.9 \mathrm{~Hz}\right.$, H-5C), $4.51\left(\mathrm{dd}, 1 \mathrm{H}, J_{5,6 \mathrm{a}}=3.2 \mathrm{~Hz}, J_{6 \mathrm{a}, 6 \mathrm{~b}}=12.5 \mathrm{~Hz}, \mathrm{H}-6 \mathrm{aD}\right), 4.32(\mathrm{~m}, 2 \mathrm{H}, \mathrm{H}-6 \mathrm{aB}$, H-6bB), 4.13-4.06 (m, 3H, H-4A, H-4C, H-6bD), 3.99-3.93 (m, 4H, H-3A, H-3C, H-4B, H-5B), 3.85-3.76 (m, 2H, H-5D, $\left.\mathrm{CH}_{2}-\mathrm{O}\right), 3.76-3.71$ (m, 4H, H-3D, COOMe), $3.68\left(\mathrm{dd}, 1 \mathrm{H}, J_{2,3}=10.2 \mathrm{~Hz}, J_{3,4}=8.7 \mathrm{~Hz}, \mathrm{H}-3 \mathrm{~B}\right), 3.64-3.57\left(\mathrm{~m}, 4 \mathrm{H}, \mathrm{COOMe}, \mathrm{CH}_{2}-\mathrm{O}\right)$, 3.48-3.36 (m, 3H, H-4D, $\left.\mathrm{CH}_{2}-\mathrm{N}\right), 3.29\left(\mathrm{dd}, 1 \mathrm{H}, J_{1,2}=3.6 \mathrm{~Hz}, J_{2,3}=10.2 \mathrm{~Hz}, \mathrm{H}-2 \mathrm{D}\right)$, 
$3.23\left(\mathrm{dd}, 1 \mathrm{H}, J_{1,2}=3.6 \mathrm{~Hz}, \mathrm{H}-2 \mathrm{~B}\right), 2.90(\mathrm{~m}, 1 \mathrm{H}, \mathrm{OH}), 2.11,2.06\left(2 \mathrm{~s}, 6 \mathrm{H}, \mathrm{CH}_{3}(\mathrm{Ac})\right)$, 1.22, 1.19 (2s, 18H, $\left.\left(\mathrm{CH}_{3}\right)_{3}(\mathrm{Piv})\right) ;{ }^{13} \mathrm{C}-\mathrm{NMR}\left(75 \mathrm{MHz}, \mathrm{CDCl}_{3}\right): \delta$ 177.6, 177.3, 172.0, 170.9, 169.9, 169.7 (CO(Ac, Piv, COOMe)), 156.5 (CO(Z)), 137.9-136.7 (Ar-C), 128.8-127.6 (Ar-CH), 99.2, 99.0, (C-1A, C-1B or C-1D), 98.1, 98.0 (C-1C, C-1B or C-1D), 78.7 (C-3B), 77.9 (C-3D), 76.0 (C-3C, C-3A), 75.0 (C-4B, $\left.\mathrm{CH}_{2}(\mathrm{OBn})\right)$, 74.0, 73.7 (C-4C, C-4A), 73.5 ( $\left.\mathrm{CH}_{2}(\mathrm{OBn})\right), 71.0$ (C-5D), 70.8 (C-2C, C-5C), 70.4 (C-2A, C-4D), 70.2 (C-5A), 69.7 (C-5B), $68.2\left(\mathrm{CH}_{2}-\mathrm{O}\right), 66.7\left(\mathrm{CH}_{2}(\mathrm{Z})\right), 62.8(\mathrm{C}-2 \mathrm{~B}, \mathrm{C}-2 \mathrm{D})$, 62.4 (C-6D), 62.0 (C-6B), 52.2, 51.8 (COOMe), $40.8\left(\mathrm{CH}_{2}-\mathrm{N}\right), 39.0(\mathrm{C}(\mathrm{Piv})), 27.3,27.2$ $\left(\left(\mathrm{CH}_{3}\right)_{3}(\mathrm{Piv})\right), 20.9\left(\mathrm{CH}_{3}(\mathrm{Ac})\right)$; HR MS: $m / z$ : calcd for $\mathrm{C}_{78} \mathrm{H}_{95} \mathrm{~N}_{7} \mathrm{O}_{27} \mathrm{Na}$ : 1584.6174; found: $1584.6115[M+\mathrm{Na}]^{+}$.

\section{$\mathrm{N}$-Benzyloxycarbonyl-2-aminoethyl (6-O-acetyl-2-azido-3-O-benzyl-}

2-deoxy-4-O-levulinoyl- $\alpha$-D-glucopyranosyl)-(1 $\rightarrow 4)$-(methyl

3-O-benzyl-2-Opivaloyl- $\alpha$-L-idopyranosyluronate)-( $1 \rightarrow 4)$-(6-O-acetyl-2-azido-3- $O$-benzyl-2-deoxy$\alpha$-D-glucopyranosyl)-( $\rightarrow 4)-($ methyl 3-O-benzyl-2- $O$-pivaloyl- $\alpha-\mathrm{L}-$ idopyranosyluronate)-(1 $\rightarrow 4)$-(6-O-acetyl-2-azido-3- $O$-benzyl-2-deoxy- $\alpha$-Dglucopyranosyl)-(1 $\rightarrow 4)$-methyl $\quad 3-O$-benzyl-2- $O$-pivaloyl- $\alpha$-L-idopyranosiduronate (13): TMSOTf $\left(157 \mu \mathrm{L}\right.$ of a $0.18 \mathrm{M}$ solution in dry $\left.\mathrm{CH}_{2} \mathrm{Cl}_{2}\right)$ was added under an argon atmosphere at $0^{\circ} \mathrm{C}$ to a mixture of $12(135 \mathrm{mg}, 0.086 \mathrm{mmol})$ and $4(133 \mathrm{mg}, 0.14 \mathrm{mmol})$ in dry $\mathrm{CH}_{2} \mathrm{Cl}_{2}(1.0 \mathrm{~mL})$. After stirring for $20 \mathrm{~min}$ at $0^{\circ} \mathrm{C}$, the reaction mixture was neutralized with $\mathrm{Et}_{3} \mathrm{~N}$ and concentrated to dryness. The residue was purified by column chromatography (hexane-EtOAc 2:1 $\rightarrow 3: 2)$ to afford $13(123 \mathrm{mg}, 61 \%)$, unreacted 12 (38 mg, 28\%) and 11 (30 mg, $0.039 \mathrm{mmol}$ ). TLC (hexane-EtOAc 1:1) $\mathrm{R}_{\mathrm{f}} 0.22 ;[\alpha]_{\mathrm{D}}^{20}$ $+5^{\circ}\left(c\right.$ 1.1, $\left.\mathrm{CH}_{2} \mathrm{Cl}_{2}\right) ;{ }^{1} \mathrm{H}-\mathrm{NMR}\left(500 \mathrm{MHz}, \mathrm{CDCl}_{3}\right): \delta$ 7.37-7.26 (m, $\left.35 \mathrm{H}, \mathrm{Ar}\right), 5.37$ (d, $\left.1 \mathrm{H}, J_{1,2}=6.1 \mathrm{~Hz}, \mathrm{H}-1 \mathrm{E}\right), 5.28\left(\mathrm{~d}, 1 \mathrm{H}, J_{1,2}=5.1 \mathrm{~Hz}, \mathrm{H}-1 \mathrm{C}\right), 5.16(\mathrm{bt}, 1 \mathrm{H}, \mathrm{NH}), 5.12(\mathrm{~d}$, $\left.1 \mathrm{H}, J_{1,2}=4.7 \mathrm{~Hz}, \mathrm{H}-1 \mathrm{~A}\right), 5.10-5.08\left(\mathrm{~m}, 3 \mathrm{H}, \mathrm{H}-4 \mathrm{~F}, \mathrm{CH}_{2}(\mathrm{Z})\right)$, 5.06-5.03 (m, 3H, H-1B, 
H-1D, H-1F), 5.00 (t, 1H, $\left.J_{2,3}=6.4 \mathrm{~Hz}, \mathrm{H}-2 \mathrm{E}\right), 4.99$ (t, 1H, $\left.J_{2,3}=5.5 \mathrm{~Hz}, \mathrm{H}-2 \mathrm{C}\right)$, 4.95-4.90 (m, 3H, H-2A, $\left.\mathrm{CH}_{2}(\mathrm{OBn})\right)$, 4.85-4.64 (m, 11H, $\mathrm{CH}_{2}(\mathrm{OBn}), \mathrm{H}-5 \mathrm{~A}$ (4.65)), $4.62\left(\mathrm{~d}, 1 \mathrm{H}, J_{4,5}=4.5 \mathrm{~Hz}, \mathrm{H}-5 \mathrm{C}\right), 4.50\left(\mathrm{~d}, 1 \mathrm{H}, J_{4,5}=5.7 \mathrm{~Hz}, \mathrm{H}-5 \mathrm{E}\right), 4.35-4.27(\mathrm{~m}, 4 \mathrm{H}$, H-6aB, H-6bB, H-6aD, H-6bD), 4.16 (dd, 1H, $\left.J_{5,6 \mathrm{a}}=4.2 \mathrm{~Hz}, J_{6 \mathrm{a}, 6 \mathrm{~b}}=12.6 \mathrm{~Hz}, \mathrm{H}-6 \mathrm{aF}\right)$, $4.11\left(\mathrm{dd}, 1 \mathrm{H}, J_{3,4}=J_{4,5}=5.4 \mathrm{~Hz}, \mathrm{H}-4 \mathrm{~A}\right), 4.07\left(\mathrm{dd}, 1 \mathrm{H}, J_{3,4}=J_{4,5}=5.4 \mathrm{~Hz}, \mathrm{H}-4 \mathrm{C}\right), 4.03(\mathrm{~m}$, 1H, H-4E), $4.01\left(\mathrm{dd}, 1 \mathrm{H}, J_{5,6 \mathrm{~b}}=2.1 \mathrm{~Hz}, \mathrm{H}-6 \mathrm{bF}\right), 3.96-3.88(\mathrm{~m}, 8 \mathrm{H}, \mathrm{H}-3 \mathrm{~A}, \mathrm{H}-3 \mathrm{C}, \mathrm{H}-3 \mathrm{E}$, H-4B, H-4D, H-5B, H-5D, H-5F), 3.84-3.78 (m, 2H, H-3F, $\left.\mathrm{CH}_{2}-\mathrm{O}\right), 3.75$ (s, 3H, COOMe), $3.71\left(\mathrm{dd}, 1 \mathrm{H}, J_{2,3}=10.4 \mathrm{~Hz}, J_{3,4}=8.4 \mathrm{~Hz}, \mathrm{H}-3 \mathrm{~B}\right.$ or H-3D), $3.63\left(\mathrm{dd}, 1 \mathrm{H}, J_{2,3}=\right.$ $10.3 \mathrm{~Hz}, J_{3,4}=8.7 \mathrm{~Hz}, \mathrm{H}-3 \mathrm{~B}$ or H-3D), $3.61\left(\mathrm{~m}, 4 \mathrm{H}, \mathrm{COOMe}, \mathrm{CH}_{2}-\mathrm{O}\right), 3.60(\mathrm{~s}, 3 \mathrm{H}$, COOMe), 3.44-3.34 (m, 2H, $\left.\mathrm{CH}_{2}-\mathrm{N}\right), 3.36\left(\mathrm{dd}, 1 \mathrm{H}, J_{2,3}=10.2 \mathrm{~Hz}, \mathrm{H}-2 \mathrm{~F}\right), 3.32-3.28$ (2dd, 2H, H-2B and H-2D), 2.74-2.65 (m, 2H, $\mathrm{CH}_{2}(\mathrm{Lev})$ ), 2.53-2.35 (m, 2H, $\left.\mathrm{CH}_{2}(\mathrm{Lev})\right), 2.16$ (s, 3H, $\left.\mathrm{CH}_{3}(\mathrm{Lev})\right), 2.11,2.08,1.99$ (3s, 9H, $\left.\mathrm{CH}_{3}(\mathrm{Ac})\right), 1.21,1.19$ (3s, 27H, $\left.\left(\mathrm{CH}_{3}\right)_{3}(\mathrm{Piv})\right) ;{ }^{13} \mathrm{C}-\mathrm{NMR}\left(125 \mathrm{MHz}, \mathrm{CDCl}_{3}\right): \delta 206.2(\mathrm{CO}(\mathrm{Lev})), 177.6,177.3$, 177.2, 170.5-169.6 (CO(Lev, Ac, Piv, COOMe)), 156.6 (CO(Z)), 137.9-136.7 (Ar-C), 128.6-127.3 (Ar-CH), 99.2 (C-1A), 98.4, 98.3, 98.1 (C-1B, C-1D, C-1F), 97.9 (C-1C), 97.7 (C-1E), 78.1, 77.9 (C-3B, C-3D), 77.5 (C-3F), 77.0-75.8 (C-3A, C-3C, C-3E), 75.2 $\left(\mathrm{CH}_{2}(\mathrm{OBn})\right), 74.9$ (C-4B, C-4D), $74.8\left(\mathrm{CH}_{2}(\mathrm{OBn})\right), 74.3,73.8\left(\mathrm{CH}_{2}(\mathrm{OBn})\right), 74.2$ (C-4C), 74.0 (C-4E), 73.6 (C-4A), 71.5 (C-2E, C-5E), 71.1 (C-2C), 70.8 (C-5C), 70.6 (C-2A), 70.5 (C-5A), 69.8 (C-5B, C-5D, $\left.\mathrm{CH}_{2}(\mathrm{Z})\right), 69.0(\mathrm{C}-5 \mathrm{~F}), 68.5\left(\mathrm{CH}_{2}-\mathrm{O}\right), 66.9$ (C4F), 63.0 (C-2B, C-2D), 62.9 (C-2F), 62.0 (C-6B, C-6D), 61.7 (C-6F), 52.5, 52.1 (COOMe), $40.9\left(\mathrm{CH}_{2}-\mathrm{N}\right), 39.0(\mathrm{C}(\mathrm{Piv})), 37.9\left(\mathrm{CH}_{2}(\mathrm{Lev})\right), 29.9\left(\mathrm{CH}_{3}(\mathrm{Lev})\right), 27.9$ $\left(\mathrm{CH}_{2}(\mathrm{Lev})\right)$ 27.3, $27.2\left(\left(\mathrm{CH}_{3}\right)_{3}(\mathrm{Piv})\right), 20.9,20.8\left(\mathrm{CH}_{3}(\mathrm{Ac})\right)$; ESI MS: m/z: calcd for $\mathrm{C}_{117} \mathrm{H}_{142} \mathrm{~N}_{10} \mathrm{O}_{41} \mathrm{Na}$ : 2365.9; found: $2366.4[M+\mathrm{Na}]^{+}$.

\section{$\mathrm{N}$-Benzyloxycarbonyl-2-aminoethyl (6-O-acetyl-2-azido-3-O-benzyl-}


idopyranosyluronate)-(1 $\rightarrow 4)-(6-O$-acetyl-2-azido-3- $O$-benzyl-2-deoxy- $\alpha$-D-

glucopyranosyl)-(1 $\rightarrow 4)-($ methyl

3-O-benzyl-2- $O$-pivaloyl- $\alpha-\mathrm{L}-$

idopyranosyluronate)-(1 $\rightarrow 4)$-(6-O-acetyl-2-azido-3- $O$-benzyl-2-deoxy- $\alpha$-D-

glucopyranosyl)-(1 $\rightarrow 4)$-methyl $\quad 3-O$-benzyl-2- $O$-pivaloyl- $\alpha$-L-idopyranosiduronate

(14): Compound 13 (119 mg, $0.051 \mathrm{mmol})$ was dissolved in $\mathrm{CH}_{2} \mathrm{Cl}_{2}(1.0 \mathrm{~mL})$ and hydrazine monohydrate $(0.197 \mathrm{~mL}$ of a $0.5 \mathrm{M}$ solution in $\mathrm{Py} / \mathrm{AcOH} 3: 2)$ was added. After stirring at room temperature for $2 \mathrm{~h}$, the reaction mixture was quenched with acetone $(0.5 \mathrm{~mL})$. The mixture was diluted with $\mathrm{CH}_{2} \mathrm{Cl}_{2}$ and washed with $\mathrm{H}_{2} \mathrm{O}$. The organic layer was dried $\left(\mathrm{MgSO}_{4}\right)$, filtered and concentrated in vacuo. The residue was purified by column chromatography (hexane-EtOAc 3:2) to yield 14 (100 mg, 87\%). TLC (hexane-EtOAc 1:1) $\mathrm{R}_{\mathrm{f}}$ 0.33; ${ }^{1} \mathrm{H}-\mathrm{NMR}\left(500 \mathrm{MHz}, \mathrm{CDCl}_{3}\right): \delta 7.40-7.25(\mathrm{~m}, 35 \mathrm{H}$, Ar), $5.38\left(\mathrm{~d}, 1 \mathrm{H}, J_{1,2}=6.1 \mathrm{~Hz}, \mathrm{H}-1 \mathrm{E}\right), 5.31\left(\mathrm{~d}, 1 \mathrm{H}, J_{1,2}=5.5 \mathrm{~Hz}, \mathrm{H}-1 \mathrm{C}\right), 5.16$ (bt, $1 \mathrm{H}$, $\mathrm{NH}), 5.12\left(\mathrm{~d}, 1 \mathrm{H}, J_{1,2}=4.8 \mathrm{~Hz}, \mathrm{H}-1 \mathrm{~A}\right), 5.12\left(\mathrm{~m}, 2 \mathrm{H}, \mathrm{CH}_{2}(\mathrm{Z})\right), 5.04-5.02(\mathrm{~m}, 3 \mathrm{H}, \mathrm{H}-1 \mathrm{~B}$, H-1D, H-1F), 5.02-4.98 (m, 2H, H-2E, H-2C), 4.96-4.92 (m, 3H, H-2A, $\mathrm{CH}_{2}(\mathrm{OBn})$ ), 4.87-4.68 (m, 10H, $\left.\mathrm{CH}_{2}(\mathrm{OBn})\right), 4.65\left(\mathrm{~d}, 1 \mathrm{H}, J_{4,5}=4.6 \mathrm{~Hz}, \mathrm{H}-5 \mathrm{~A}\right), 4.58\left(\mathrm{~d}, 1 \mathrm{H}, J_{4,5}=5.1\right.$ Hz, H-5C), 4.53-4.48 (m, 2H, H-6aF, H-5E), 4.36-4.26 (m, 4H, H-6aB, H-6bB, H-6aD, H-6bD), 4.13-4.02 (m, 4H, H-6bF, H-4A, H-4C, H-4E), 3.99-3.87 (m, 8H, H-3A, H-3C, H-3E, H-4B, H-4D, H-5B, H-5D, H-5F), 3.84-3.56 (m, 14H, H-3F, H-3B, H-3D, $\mathrm{CH}_{2}-\mathrm{O}$, COOMe $(3.79,3.62)$ ), 3.47-3.33 (m, 3H, H-4F, $\left.\mathrm{CH}_{2}-\mathrm{N}\right), 3.33-3.22$ (3dd, 3H, H-2F, H-2B, H-2D), 2.93 (bd, 1H, OH), 2.11, 2.07, 2.06 (3s, 9H, $\left.\mathrm{CH}_{3}(\mathrm{Ac})\right), 1.20,1.19$ $\left(3 \mathrm{~s}, 27 \mathrm{H},\left(\mathrm{CH}_{3}\right)_{3}(\mathrm{Piv})\right) ;{ }^{13} \mathrm{C}-\mathrm{NMR}\left(125 \mathrm{MHz}, \mathrm{CDCl}_{3}\right): \delta 177.6,177.3,177.2,172.0$, 170.9, 170.8, 170.0, 169.9, 169.7 (CO(Ac, Piv, COOMe)), $156.5(\mathrm{CO}(\mathrm{Z}))$, 137.9-136.6 (Ar-C), 128.8-127.2 (Ar-CH), 99.1 (C-1A), 99.0, 98.3, 98.0 (C-1B, C-1D, C-1F), 97.8 (C-1C), 97.7 (C-1E), 79.0 (C-3F), 78.1, 77.9 (C-3B, C-3D), 76.9-76.4 (C-3A, C-3C, 
C-3E), $75.3\left(\mathrm{CH}_{2}(\mathrm{OBn}), \mathrm{C}-4 \mathrm{~B}\right.$ or C-4D), 75.0 (C-4B or C-4D), $74.9\left(\mathrm{CH}_{2}(\mathrm{OBn})\right), 74.4$, 73.9 (C-4A or $\mathrm{C}-4 \mathrm{C}$ or $\mathrm{C}-4 \mathrm{E}), 73.8\left(\mathrm{CH}_{2}(\mathrm{OBn})\right), 73.6(\mathrm{C}-4 \mathrm{~A}$ or $\mathrm{C}-4 \mathrm{C}$ or $\mathrm{C}-4 \mathrm{E}$, $\left.\mathrm{CH}_{2}(\mathrm{OBn})\right), 71.6$ (C-5E, C-2C or C-2E), 71.3 (C-2C or C-2E), 71.2 (C-5C, C-5F), 70.5 (C-2A, C-5A, C-4F), 69.8 (C-5B, C-5D), $68.5\left(\mathrm{CH}_{2}-\mathrm{O}\right), 66.8\left(\mathrm{CH}_{2}(\mathrm{Z})\right), 62.9(\mathrm{C}-2 \mathrm{~B}$, C-2D, C-2F), 62.6 (C-6F), 62.0 (C-6B, C-6D), 52.5, 52.1 (COOMe), $40.9\left(\mathrm{CH}_{2}-\mathrm{N}\right)$, 39.0 (C(Piv)), 27.3, $27.2\left(\left(\mathrm{CH}_{3}\right)_{3}(\mathrm{Piv})\right), 20.9\left(\mathrm{CH}_{3}(\mathrm{Ac})\right)$; ESI MS: $m / z$ : calcd for $\mathrm{C}_{112} \mathrm{H}_{136} \mathrm{~N}_{10} \mathrm{O}_{39} \mathrm{Na}$ : 2267.9; found: $2268.3[M+\mathrm{Na}]^{+}$.

\section{Methyl ( $\mathrm{N}$-benzyloxycarbonyl-2-aminoethyl $\quad$ 4- $O$-(6- $O$-acetyl-2-azido-3,4-di- $O$ -} benzyl-2-deoxy- $\alpha$-D-glucopyranosyl)-3- $O$-benzyl-2- $O$-pivaloyl- $\alpha$-L-idopyranoside)

uronate (15): Benzyl bromide $(40 \mu \mathrm{L}, 0.34 \mathrm{mmol})$ was added under an argon atmosphere at $0^{\circ} \mathrm{C}$ to a solution of $9(30 \mathrm{mg}, 0.034 \mathrm{mmol})$ and freshly prepared $\mathrm{Ag}_{2} \mathrm{O}$ (18 $\mathrm{mg}, 0.079 \mathrm{mmol})$ in dry DMF $(0.5 \mathrm{~mL})$. After stirring for $8 \mathrm{~h}$ at room temperature, the solution was filtered through Celite, diluted with EtOAc and washed with $\mathrm{H}_{2} \mathrm{O}$. The organic layer was dried $\left(\mathrm{MgSO}_{4}\right)$, filtered and concentrated in vacuo. The residue was purified by column chromatography (hexane-EtOAc $1: 0 \rightarrow 2: 1)$ to afford $15(8 \mathrm{mg}$, $25 \%)$ and unreacted $9(8 \mathrm{mg}, 27 \%)$. TLC (hexane-EtOAc 3:2) $\mathrm{R}_{\mathrm{f}} 0.4 ;[\alpha]_{\mathrm{D}}^{20}+8^{\circ}(c 1.0$, $\mathrm{CHCl}_{3}$ ); ${ }^{1} \mathrm{H}-\mathrm{NMR}\left(300 \mathrm{MHz}, \mathrm{CDCl}_{3}\right): \delta 7.39-7.25$ (m, 20H, Ar), 5.18 (bt, $1 \mathrm{H}, \mathrm{NH}$ ), $5.09\left(\mathrm{~m}, 3 \mathrm{H}, \mathrm{H}-1 \mathrm{~A}, \mathrm{CH}_{2}(\mathrm{Z})\right), 5.05\left(\mathrm{~d}, 1 \mathrm{H}, J_{1,2}=3.6 \mathrm{~Hz}, \mathrm{H}-1 \mathrm{~B}\right), 4.95\left(\mathrm{t}, 1 \mathrm{H}, J_{1,2}=J_{2,3}=\right.$ $4.8 \mathrm{~Hz}, \mathrm{H}-2 \mathrm{~A}), 4.85\left(\mathrm{~d}, 1 \mathrm{H}, \mathrm{CH}_{2}(\mathrm{OBn})\right), 4.83$ (bs, 2H, $\left.\mathrm{CH}_{2}(\mathrm{OBn})\right), 4.75$ (m, 2H, $\left.\mathrm{CH}_{2}(\mathrm{OBn})\right), 4.72\left(\mathrm{~d}, 1 \mathrm{H}, J_{4,5}=4.4 \mathrm{~Hz}, \mathrm{H}-5 \mathrm{~A}\right), 4.59$ (d, 1H, $\left.\mathrm{CH}_{2}(\mathrm{OBn})\right), 4.32$ (dd, 1H, $\left.J_{5,6 \mathrm{a}}=1.9 \mathrm{~Hz}, J_{6 \mathrm{a}, 6 \mathrm{~b}}=12.1 \mathrm{~Hz}, \mathrm{H}-6 \mathrm{aB}\right), 4.18\left(\mathrm{dd}, 1 \mathrm{H}, J_{5,6 \mathrm{~b}}=4.2 \mathrm{~Hz}, \mathrm{H}-6 \mathrm{bB}\right), 4.14(\mathrm{t}, 1 \mathrm{H}$, $\left.J_{3,4}=4.5 \mathrm{~Hz}, \mathrm{H}-4 \mathrm{~A}\right), 3.98$ (t, 1H, H-3A), 3.94 (m, 1H, H-5B), 3.87 (dd, $1 \mathrm{H}, J_{2,3}=10.3$ $\left.\mathrm{Hz}, J_{3,4}=8.9 \mathrm{~Hz}, \mathrm{H}-3 \mathrm{~B}\right), 3.81\left(\mathrm{~m}, 1 \mathrm{H}, \mathrm{CH}_{2}-\mathrm{O}\right), 3.78(\mathrm{~s}, 3 \mathrm{H}, \mathrm{COOMe}), 3.63(\mathrm{~m}, 1 \mathrm{H}$, $\mathrm{CH}_{2}-\mathrm{O}$ ), 3.52 (dd, 1H, $\left.J_{4,5}=9.9 \mathrm{~Hz}, \mathrm{H}-4 \mathrm{~B}\right), 3.40\left(\mathrm{~m}, 2 \mathrm{H}, \mathrm{CH}_{2}-\mathrm{N}\right), 3.28$ (dd, 1H, H-2B), 2.01 (s, 3H, $\left.\mathrm{CH}_{3}(\mathrm{Ac})\right), 1.20\left(\mathrm{~s}, 9 \mathrm{H},\left(\mathrm{CH}_{3}\right)_{3}(\mathrm{Piv})\right) ;{ }^{13} \mathrm{C}-\mathrm{NMR}\left(125 \mathrm{MHz}, \mathrm{CDCl}_{3}\right): \delta$ 
177.6, 170.6, 169.7 (CO(Ac, Piv, COOMe)), 156.5 (CO(Z)), 137.8-136.6 (Ar-C), 128.7127.6 (Ar-CH), 99.2 (C-1A), 98.3 (C-1B), 80.0 (C-3B), 77.8 (C-4B), 75.8 (C-3A), 75.5, $74.9\left(\mathrm{CH}_{2}(\mathrm{OBn})\right), 73.8(\mathrm{C}-4 \mathrm{~A}), 73.4\left(\mathrm{CH}_{2}(\mathrm{OBn})\right), 70.4,70.1$ (C-2A, C-5A, C-5B), 68.5 $\left(\mathrm{CH}_{2}-\mathrm{O}\right), 66.8\left(\mathrm{CH}_{2}(\mathrm{Z})\right), 63.4(\mathrm{C}-2 \mathrm{~B}), 62.6(\mathrm{C}-6 \mathrm{~B}), 52.5(\mathrm{COOMe}), 40.9\left(\mathrm{CH}_{2}-\mathrm{N}\right), 38.9$ (C(Piv)), $27.2\left(\left(\mathrm{CH}_{3}\right)_{3}(\mathrm{Piv})\right), 20.9\left(\mathrm{CH}_{3}(\mathrm{Ac})\right)$; HR MS: $m / z$ : calcd for $\mathrm{C}_{51} \mathrm{H}_{60} \mathrm{~N}_{4} \mathrm{O}_{15} \mathrm{Na}$ : 991.3953; found: $991.3984[M+\mathrm{Na}]^{+}$.

Methyl 4-O-(6-O-acetyl-2-azido-3,4-di- $O$-benzyl-2-deoxy- $\alpha$-D-glucopyranosyl)-3- $O$ benzyl-2- $\boldsymbol{O}$-pivaloyl-a, $\boldsymbol{\beta}$-L-idopyranosuronate (17): TMSOTf $(22 \mu \mathrm{L}$ of a $0.18 \mathrm{M}$ solution in dry $\left.\mathrm{CH}_{2} \mathrm{Cl}_{2}\right)$ was added to a cooled $\left(0^{\circ} \mathrm{C}\right)$ solution of $2(1.21 \mathrm{~g}, 2.75 \mathrm{mmol})$ in dry $\mathrm{CH}_{2} \mathrm{Cl}_{2}(30 \mathrm{~mL})$ under an argon atmosphere. While the reaction was stirred, a solution of $16(1.31 \mathrm{~g}, 2.29 \mathrm{mmol})$ in dry $\mathrm{CH}_{2} \mathrm{Cl}_{2}(10 \mathrm{~mL})$ was added dropwise. After 20 min at $0^{\circ} \mathrm{C}$, the mixture was neutralized with $\mathrm{Et}_{3} \mathrm{~N}$ and concentrated in vacuo. The residue was separated by flash column chromatography (toluene/EtOAc 12:1) to obtain the desired $\alpha(1 \rightarrow 4)$ disaccharide. This compound was dissolved in Py $(15 \mathrm{~mL})$. Pivaloyl chloride (5 mL) and DMAP (cat.) were added and the solution was stirred at room temperature. After $24 \mathrm{~h}$, the mixture was diluted with $\mathrm{CH}_{2} \mathrm{Cl}_{2}$, washed with $1 \mathrm{M}$ $\mathrm{HCl}$ aqueous solution, saturated $\mathrm{NaHCO}_{3}$ aqueous solution and $\mathrm{H}_{2} \mathrm{O}$, dried $\left(\mathrm{MgSO}_{4}\right)$, filtered, and concentrated in vacuo. The residue was purified by flash column chromatography (toluene/EtOAc 49:1) to yield the desired 2-O-pivaloylated disaccharide (1.1 g, 47\%, 2 steps). TLC (toluene-EtOAc 12:1) $\mathrm{R}_{\mathrm{f}}$ 0.43; ${ }^{1} \mathrm{H}-\mathrm{NMR}(500$ $\mathrm{MHz}_{\mathrm{CDCl}}$ ): $\delta$ 7.39-7.25 (m, 15H, Ar), 5.04 (bs, 2H, H-1A, H-2A), $4.94\left(\mathrm{~d}, 1 \mathrm{H}, J_{1,2}=\right.$ $3.5 \mathrm{~Hz}, \mathrm{H}-1 \mathrm{~B}), 4.85-4.58\left(\mathrm{~m}, 6 \mathrm{H}, \mathrm{CH}_{2}(\mathrm{OBn})\right), 4.42(\mathrm{bd}, 1 \mathrm{H}, \mathrm{H}-5 \mathrm{~A}), 4.35$ (dd, $1 \mathrm{H}, J_{5,6 \mathrm{a}}=$ $\left.1.9 \mathrm{~Hz}, J_{6 \mathrm{a}, 6 \mathrm{~b}}=12.3 \mathrm{~Hz}, \mathrm{H}-6 \mathrm{aB}\right), 4.20\left(\mathrm{dd}, 1 \mathrm{H}, J_{5,6 \mathrm{~b}}=2.8 \mathrm{~Hz}, \mathrm{H}-6 \mathrm{bB}\right), 4.09-4.04(\mathrm{~m}, 2 \mathrm{H}$, H-5B, H-4A), 4.01-3.97 (m, 2H, H-3A, H-3B), 3.74 (s, 3H, COOMe), 3.57 (t, 1H, $J_{4,5}=$ $9.5 \mathrm{~Hz}, \mathrm{H}-4 \mathrm{~B}), 3.28$ (dd, 1H, $\left.J_{2,3}=10.3 \mathrm{~Hz}, \mathrm{H}-2 \mathrm{~B}\right), 2.00$ (s, 3H, $\mathrm{CH}_{3}(\mathrm{Ac})$ ), 1.63 (hp, 1H, 
$\left.\mathrm{CH}\left(\mathrm{CH}_{3}\right)_{2}\right), 1.27\left(\mathrm{~s}, 9 \mathrm{H}, \mathrm{C}\left(\mathrm{CH}_{3}\right)_{3}\right), 0.88-0.85\left(12 \mathrm{H}, \mathrm{CH}\left(\mathrm{CH}_{3}\right)_{2}, \mathrm{C}\left(\mathrm{CH}_{3}\right)_{2}\right), 0.20,0.15(2 \mathrm{~s}$, 6H, $\left.\mathrm{Si}\left(\mathrm{CH}_{3}\right)_{2}\right) ;{ }^{13} \mathrm{C}-\mathrm{NMR} \quad\left(125 \mathrm{MHz}, \mathrm{CDCl}_{3}\right)$ (Significant data from HMQC experiment): $\delta 98.8$ (C-1B), 94.5 (C-1A), 80.2 (C-3B), 77.5 (C-4B), 75.6 (C-3A), 75.4 $\left(\mathrm{CH}_{2}(\mathrm{OBn})\right), 74.7\left(\mathrm{CH}_{2}(\mathrm{OBn})\right), 74.4$ (C-4A), 73.4 (C-5A), $73.0\left(\mathrm{CH}_{2}(\mathrm{OBn})\right), 69.8$ (C-5B), 67.7 (C-2A), 63.5 (C-2B), 62.3 (C-6B), 52.0 (COOMe); ESI MS: m/z: calcd for $\mathrm{C}_{49} \mathrm{H}_{67} \mathrm{~N}_{3} \mathrm{O}_{13} \mathrm{SiNa}$ : 956.4; found: $956.0[M+\mathrm{Na}]^{+}$.

To a solution of this $2-O$-pivaloylated disaccharide $(0.8 \mathrm{~g}, 0.86 \mathrm{mmol})$ at $-15^{\circ} \mathrm{C}$ in dry THF $(23 \mathrm{~mL})$, an excess of $(\mathrm{HF})_{\mathrm{n}} \cdot \mathrm{Py}(2.4 \mathrm{~mL})$ was added. The reaction was warmed to $0^{\circ} \mathrm{C}$ and stirred for $28 \mathrm{~h}$ under an argon atmosphere. Then, the reaction was stirred at room temperature until complete disappearance of starting material. The mixture was diluted with $\mathrm{CH}_{2} \mathrm{Cl}_{2}$ and washed with $\mathrm{H}_{2} \mathrm{O}$ and saturated $\mathrm{NaHCO}_{3}$ solution until neutral $\mathrm{pH}$. The organic layer was dried $\left(\mathrm{MgSO}_{4}\right)$, filtered, and concentrated in vacuo to give 17 (615 mg, 91\%). TLC (hexane-EtOAc 2:1) $\mathrm{R}_{\mathrm{f}} 0.29 ;{ }^{1} \mathrm{H}-\mathrm{NMR}(300$ $\mathrm{MHz}, \mathrm{CDCl}_{3}$ ) (data for major anomer): $\delta 7.37-7.25\left(\mathrm{~m}, 15 \mathrm{H}, \mathrm{Ar}\right.$ ), 5.38 (bd, $1 \mathrm{H}, J_{1,2}=$ $4.8 \mathrm{~Hz}, \mathrm{H}-1 \mathrm{~A}), 5.06$ (d, 1H, $\left.J_{1,2}=3.6 \mathrm{~Hz}, \mathrm{H}-1 \mathrm{~B}\right), 4.89-4.78$ (m, 7H, H-2A, H-5A, $\left.\mathrm{CH}_{2}(\mathrm{OBn})\right), 4.6\left(\mathrm{~m}, 1 \mathrm{H}, \mathrm{CH}_{2}(\mathrm{OBn})\right), 4.33\left(\mathrm{dd}, 1 \mathrm{H}, J_{5,6 \mathrm{a}}=2.1 \mathrm{~Hz}, J_{6 \mathrm{a}, 6 \mathrm{~b}}=12.0 \mathrm{~Hz}\right.$, H-6aB), 4.21-4.10 (m, 3H, H-6bB, H-3A, H-4A), 3.95 (ddd, $1 \mathrm{H}, J_{4,5}=10.0 \mathrm{~Hz}, J_{5,6 \mathrm{~b}}=$ 4.1 Hz,H-5B), 3.85 (dd, 1H, $\left.J_{2,3}=10.2 \mathrm{~Hz}, J_{3,4}=8.8 \mathrm{~Hz}, \mathrm{H}-3 \mathrm{~B}\right), 3.80$ (s, 3H, COOMe), $3.51(\mathrm{dd}, 1 \mathrm{H}, \mathrm{H}-4 \mathrm{~B}), 3.30$ (dd, 1H, H-2B), 2.02 (s, 3H, $\left.\mathrm{CH}_{3}(\mathrm{Ac})\right), 1.24$ (s, 9H, $\left.\left(\mathrm{CH}_{3}\right)_{3}(\mathrm{Piv})\right) ;{ }^{13} \mathrm{C}-\mathrm{NMR}\left(75 \mathrm{MHz}, \mathrm{CDCl}_{3}\right)$ (selected data for major anomer from HMQC experiment): $\delta 99.0$ (C-1B), 93.6 (C-1A), 80.0 (C-3B), 77.7 (C-4B), 75.4, 74.1 (C-3A, C-4A), 70.1 (C-5A), 70.0 (C-5B), 63.3 (C-2B), 62.4 (C-6B), 52.4 (COOMe), 27.2 (( $\left.\left.\mathrm{CH}_{3}\right)_{3}(\mathrm{Piv})\right), 20.8\left(\mathrm{CH}_{3}(\mathrm{Ac})\right) ;{ }^{1} \mathrm{H}-\mathrm{NMR}\left(300 \mathrm{MHz}, \mathrm{CDCl}_{3}\right)$ (selected data for minor anomer): $\delta$ 7.37-7.25 (m, 15H, Ar), $5.23(\mathrm{bd}, 1 \mathrm{H}, \mathrm{H}-1 \mathrm{~A}), 4.99\left(\mathrm{~d}, 1 \mathrm{H}, J_{1,2}=3.6 \mathrm{~Hz}\right.$, H-1B), 4.94-4.57 (m, 8H, H-2A, H-5A, $\mathrm{CH}_{2}(\mathrm{OBn})$ ), 3.80 (s, 3H, COOMe), 3.34 (dd, 
1H, H-2B), 2.02 (s, 3H, $\left.\mathrm{CH}_{3}(\mathrm{Ac})\right), 1.24$ (s, 9H, $\left.\left(\mathrm{CH}_{3}\right)_{3}(\mathrm{Piv})\right)$; HR MS: m/z: calcd for $\mathrm{C}_{41} \mathrm{H}_{49} \mathrm{~N}_{3} \mathrm{O}_{13} \mathrm{Na}$ : 814.3163; found: $814.3159[M+\mathrm{Na}]^{+}$.

$O$-(Methyl 4-O-(6-O-acetyl-2-azido-3,4-di- $O$-benzyl-2-deoxy- $\alpha$-D-glucopyranosyl)3-O-benzyl-2-O-pivaloyl- $\alpha, \beta$-L-idopyranosyluronate) trichloroacetimidate $\quad(18)$ : $\mathrm{K}_{2} \mathrm{CO}_{3}(13 \mathrm{mg}, 96 \mu \mathrm{mol})$ and trichloroacetonitrile $(119 \mu \mathrm{L}, 1.19 \mathrm{mmol})$ were added at room temperature to a solution of $\mathbf{1 7}(63 \mathrm{mg}, 80 \mu \mathrm{mol})$ in dry $\mathrm{CH}_{2} \mathrm{Cl}_{2}(1 \mathrm{~mL})$. After stirring for $6 \mathrm{~h}$ at room temperature, the reaction mixture was filtered through a pad of Celite and concentrated to dryness. The residue was purified by column chromatography (hexane-EtOAc 3:1 $+1 \% \mathrm{Et}_{3} \mathrm{~N}$ ) to yield $18(70 \mathrm{mg}, 94 \%)$. TLC (hexane-EtOAc 2:1) $\mathrm{R}_{\mathrm{f}}$ 0.49, 0.59 ( $\alpha$ and $\beta$ anomers); ${ }^{1} \mathrm{H}-\mathrm{NMR}\left(300 \mathrm{MHz}, \mathrm{CDCl}_{3}\right.$ ) (for major anomer): $\delta 8.71(\mathrm{~s}, 1 \mathrm{H}, \mathrm{NH}), 7.36-7.25(\mathrm{~m}, 15 \mathrm{H}, \mathrm{Ar}), 6.44\left(\mathrm{~d}, 1 \mathrm{H}, J_{1,2}=2.7 \mathrm{~Hz}\right.$, H-1A), $5.20\left(\mathrm{t}, 1 \mathrm{H}, J_{2,3}=3.3 \mathrm{~Hz}, \mathrm{H}-2 \mathrm{~A}\right), 5.03\left(\mathrm{~d}, 1 \mathrm{H}, J_{1,2}=3.5 \mathrm{~Hz}, \mathrm{H}-1 \mathrm{~B}\right), 4.91(\mathrm{~d}, 1 \mathrm{H}$, $\left.J_{4,5}=3.2 \mathrm{~Hz}, \mathrm{H}-5 \mathrm{~A}\right), 4.87-4.58\left(\mathrm{~m}, 6 \mathrm{H}, \mathrm{CH}_{2}(\mathrm{OBn})\right), 4.35\left(\mathrm{dd}, 1 \mathrm{H}, J_{5,6 \mathrm{a}}=2.0 \mathrm{~Hz}, J_{6 \mathrm{a}, 6 \mathrm{~b}}=\right.$ $12.1 \mathrm{~Hz}, \mathrm{H}-6 \mathrm{aB}), 4.23\left(\mathrm{t}, 1 \mathrm{H}, J_{3,4}=3.8 \mathrm{~Hz}, \mathrm{H}-4 \mathrm{~A}\right), 4.18$ (dd, $\left.1 \mathrm{H}, J_{5,6 \mathrm{~b}}=3.7 \mathrm{~Hz}, \mathrm{H}-6 \mathrm{bB}\right)$, 4.07 (t, 1H, H-3A), 3.95 (ddd, 1H, H-5B), $3.90\left(\mathrm{dd}, 1 \mathrm{H}, J_{2,3}=10.3 \mathrm{~Hz}, J_{3,4}=8.8 \mathrm{~Hz}\right.$, H-3B), 3.80 (s, 3H, COOMe), 3.54 (dd, 1H, J4,5 = 9.9 Hz, H-4B), 3.33 (dd, 1H, H-2B), $2.01\left(\mathrm{~s}, 3 \mathrm{H}, \mathrm{CH}_{3}(\mathrm{Ac})\right), 1.26\left(\mathrm{~s}, 9 \mathrm{H},\left(\mathrm{CH}_{3}\right)_{3}(\mathrm{Piv})\right) ;{ }^{13} \mathrm{C}-\mathrm{NMR}\left(75 \mathrm{MHz}, \mathrm{CDCl}_{3}\right)$ (selected data for major anomer from HSQC experiment): $\delta 98.4$ (C-1B), 95.5 (C-1A), 80.1 (C-3B), 77.5 (C-4B), 75.3, $74.8 \quad\left(\mathrm{CH}_{2}(\mathrm{OBn})\right), 74.2 \quad$ (C-3A), $73.7 \quad$ (C-4A), 72.8 $\left(\mathrm{CH}_{2}(\mathrm{OBn})\right), 70.1$ (C-5A, C-5B), 67.6 (C-2A), 63.3 (C-2B), 62.4 (C-6B), 52.4 (COOMe), $27.1\left(\left(\mathrm{CH}_{3}\right)_{3}(\mathrm{Piv})\right), 20.7\left(\mathrm{CH}_{3}(\mathrm{Ac})\right) ;{ }^{1} \mathrm{H}-\mathrm{NMR}\left(300 \mathrm{MHz}, \mathrm{CDCl}_{3}\right)$ (selected data for minor anomer): $\delta 8.64(\mathrm{~s}, 1 \mathrm{H}, \mathrm{NH}), 7.36-7.25(\mathrm{~m}, 15 \mathrm{H}, \mathrm{Ar}), 6.38\left(\mathrm{~d}, 1 \mathrm{H}, J_{1,2}=\right.$ $2.7 \mathrm{~Hz}, \mathrm{H}-1 \mathrm{~A}), 5.21$ (m, 1H, H-2A), 5.00 (d, 1H, $\left.J_{1,2}=3.9 \mathrm{~Hz}, \mathrm{H}-1 \mathrm{~B}\right), 4.87-4.58$ (m, 7H, $\left.\mathrm{CH}_{2}(\mathrm{OBn}), \mathrm{H}-5 \mathrm{~A}(4.66)\right), 4.40-4.29$ (m, 2H, H-3A, H-6aB), 4.25-4.04 (m, 2H, H6bB, H-4A), 3.99-3.82 (m, 2H, H-3B, H-5B), 3.78 (s, 3H, COOMe), 3.52 (m, 1H, H- 
4B), $3.38\left(\mathrm{dd}, 1 \mathrm{H}, J_{2,3}=10.3 \mathrm{~Hz}, \mathrm{H}-2 \mathrm{~B}\right), 2.01\left(\mathrm{~s}, 3 \mathrm{H}, \mathrm{CH}_{3}(\mathrm{Ac})\right), 1.26(\mathrm{~s}, 9 \mathrm{H}$, $\left.\left(\mathrm{CH}_{3}\right)_{3}(\mathrm{Piv})\right)$; ${ }^{13} \mathrm{C}-\mathrm{NMR}\left(75 \mathrm{MHz}, \mathrm{CDCl}_{3}\right.$ ) (selected data for minor anomer from HSQC experiment): $\delta 99.3$ (C-1B), 94.8 (C-1A), 80.1 (C-3B), 77.5 (C-4B), 76.1 (C-4A), 74.6 (C-3A), 70.1 (C-5B), 68.5 (C-2A), 63.6 (C-2B), 52.4 (COOMe), $27.1\left(\left(\mathrm{CH}_{3}\right)_{3}(\mathrm{Piv})\right)$, $20.7\left(\mathrm{CH}_{3}(\mathrm{Ac})\right)$; ESI MS: $m / z$ : calcd for $\mathrm{C}_{43} \mathrm{H}_{49} \mathrm{Cl}_{3} \mathrm{~N}_{4} \mathrm{O}_{13} \mathrm{Na}$ : 957.2; found: 956.7 $[M+\mathrm{Na}]^{+}$.

\section{$N$-Benzyloxycarbonyl-2-aminoethyl $O$-(6-O-acetyl-2-azido-3,4-di- $O$-benzyl-}

2-deoxy- $\alpha$-D-glucopyranosyl)-( $1 \rightarrow 4)-O$-(methyl

3-O-benzyl-2-O-pivaloyl- $\alpha-\mathrm{L}-$ idopyranosyluronate)-(1 $\rightarrow 4)-O$-(6-O-acetyl-2-azido-3-O-benzyl-2-deoxy- $\alpha$-Dglucopyranosyl)-(1 $\rightarrow 4)$-methyl $\quad 3-O$-benzyl-2- $O$-pivaloyl- $\alpha$-L-idopyranosiduronate (19): TMSOTf $\left(127 \mu \mathrm{L}\right.$ of a $0.18 \mathrm{M}$ solution in dry $\left.\mathrm{CH}_{2} \mathrm{Cl}_{2}\right)$ was added under an argon atmosphere at $0^{\circ} \mathrm{C}$ to a mixture of $\mathbf{9}(44 \mathrm{mg}, 0.05 \mathrm{mmol})$ and $18(70 \mathrm{mg}, 0.075 \mathrm{mmol})$ in dry $\mathrm{CH}_{2} \mathrm{Cl}_{2}(1.0 \mathrm{~mL})$. After stirring for $20 \mathrm{~min}$ at $0^{\circ} \mathrm{C}$, the reaction mixture was neutralized with $\mathrm{Et}_{3} \mathrm{~N}$ and concentrated to dryness. The residue was purified by column chromatography (toluene-EtOAc 5:1) to afford 19 (40 mg, 48\%) and unreacted 9 (20 $\mathrm{mg}, 45 \%)$. TLC (hexane-EtOAc 2:1) $\mathrm{R}_{\mathrm{f}} 0.26 ;[\alpha]_{\mathrm{D}}^{20}+10^{\circ}\left(c\right.$ 1.2, $\left.\mathrm{CHCl}_{3}\right) ;{ }^{1} \mathrm{H}-\mathrm{NMR}(500$ $\left.\mathrm{MHz}, \mathrm{CDCl}_{3}\right): \delta 7.37-7.23(\mathrm{~m}, 30 \mathrm{H}, \mathrm{Ar}), 5.31$ (d, $\left.1 \mathrm{H}, J_{1,2}=5.2 \mathrm{~Hz}, \mathrm{H}-1 \mathrm{C}\right), 5.16$ (bt, $1 \mathrm{H}$, $\mathrm{NH}), 5.11\left(\mathrm{~d}, 1 \mathrm{H}, J_{1,2}=4.7 \mathrm{~Hz}, \mathrm{H}-1 \mathrm{~A}\right), 5.09\left(\mathrm{bs}, 2 \mathrm{H}, \mathrm{CH}_{2}(\mathrm{Z})\right), 5.05\left(\mathrm{~d}, 1 \mathrm{H}, J_{1,2}=3.5 \mathrm{~Hz}\right.$, H-1D), $5.02\left(\mathrm{~d}, 1 \mathrm{H}, J_{1,2}=3.5 \mathrm{~Hz}, \mathrm{H}-1 \mathrm{~B}\right), 4.99\left(\mathrm{t}, 1 \mathrm{H}, J_{2,3}=5.6 \mathrm{~Hz}, \mathrm{H}-2 \mathrm{C}\right), 4.96-4.92(\mathrm{~m}$, $\left.2 \mathrm{H}, \mathrm{H}-2 \mathrm{~A}, \mathrm{CH}_{2}(\mathrm{OBn})\right), 4.86-4.68\left(\mathrm{~m}, 8 \mathrm{H}, \mathrm{CH}_{2}(\mathrm{OBn})\right), 4.67\left(\mathrm{~d}, 1 \mathrm{H}, J_{4,5}=4.6 \mathrm{~Hz}\right.$, H-5A), $4.62\left(\mathrm{~d}, 1 \mathrm{H}, J_{4,5}=5.0 \mathrm{~Hz}, \mathrm{H}-5 \mathrm{C}\right), 4.59\left(\mathrm{~d}, 1 \mathrm{H}, \mathrm{CH}_{2}(\mathrm{OBn})\right), 4.36-4.29(\mathrm{~m}, 2 \mathrm{H}$, H-6aB, H-6bB), $4.26\left(\mathrm{dd}, 1 \mathrm{H}, J_{5,6 \mathrm{a}}=1.8 \mathrm{~Hz}, J_{6 \mathrm{a}, 6 \mathrm{~b}}=12.2 \mathrm{~Hz}, \mathrm{H}-6 \mathrm{aD}\right), 4.16\left(\mathrm{dd}, 1 \mathrm{H}, J_{5,6 \mathrm{~b}}\right.$ $=3.4 \mathrm{~Hz}, \mathrm{H}-6 \mathrm{bD}), 4.11\left(\mathrm{t}, 1 \mathrm{H}, J_{3,4}=5.2 \mathrm{~Hz}, \mathrm{H}-4 \mathrm{~A}\right), 4.08\left(\mathrm{t}, 1 \mathrm{H}, J_{3,4}=5.3 \mathrm{~Hz}, \mathrm{H}-4 \mathrm{C}\right)$, 3.97-3.90 (m, 5H, H-3A, H-3C, H-4B, H-5B, H-5D), 3.84-3.80 (m, 2H, H-3D, $\mathrm{CH}_{2}-\mathrm{O}$ ), 3.75-3.70 (m, 4H, H-3B, COOMe), 3.64-3.58 (m, 4H, COOMe, $\left.\mathrm{CH}_{2}-\mathrm{O}\right), 3.54$ (t, $1 \mathrm{H}$, 
$\left.J_{3,4}=J_{4,5}=9.3 \mathrm{~Hz}, \mathrm{H}-4 \mathrm{D}\right), 3.39\left(\mathrm{~m}, 2 \mathrm{H}, \mathrm{CH}_{2}-\mathrm{N}\right), 3.29(\mathrm{~m}, 2 \mathrm{H}, \mathrm{H}-2 \mathrm{D}, \mathrm{H}-2 \mathrm{~B}), 2.10,1.96$ (2s, 6H, $\left.\mathrm{CH}_{3}(\mathrm{Ac})\right), 1.21,1.19\left(2 \mathrm{~s}, 18 \mathrm{H},\left(\mathrm{CH}_{3}\right)_{3}(\mathrm{Piv})\right) ;{ }^{13} \mathrm{C}-\mathrm{NMR}\left(125 \mathrm{MHz}, \mathrm{CDCl}_{3}\right)$ (Significant data from HSQC experiment): $\delta 99.0$ (C-1A), 98.4 (C-1D), 97.9 (C-1B), 97.8 (C-1C), 79.9 (C-3D), 77.9 (C-3B), 77.4 (C-4D), 75.9 (C-3C, C-3A), 75.3, 75.0 $\left(\mathrm{CH}_{2}(\mathrm{OBn})\right), \quad 74.9$ (C-4B), $74.7, \quad 73.8 \quad\left(\mathrm{CH}_{2}(\mathrm{OBn})\right), \quad 73.7 \quad$ (C-4C, C-4A), 73.3 $\left(\mathrm{CH}_{2}(\mathrm{OBn})\right), 70.8$ (C-2C), 70.7 (C-5C), 70.2 (C-2A), 70.1 (C-5A), 69.9 (C-5B), 69.7 (C-5D), $68.2\left(\mathrm{CH}_{2}-\mathrm{O}\right), 66.6\left(\mathrm{CH}_{2}(\mathrm{Z})\right), 63.0$ (C-2B, C-2D), 62.3 (C-6D), 61.9 (C-6B), 52.2, $51.9(\mathrm{COOMe}), 40.8\left(\mathrm{CH}_{2}-\mathrm{N}\right), 27.1\left(\left(\mathrm{CH}_{3}\right)_{3}(\mathrm{Piv})\right), 20.7\left(\mathrm{CH}_{3}(\mathrm{Ac})\right)$; HR MS: m/z: calcd for $\mathrm{C}_{85} \mathrm{H}_{101} \mathrm{~N}_{7} \mathrm{O}_{27} \mathrm{Na}$ : 1674.6643; found: $1674.6697[M+\mathrm{Na}]^{+}$.

\section{$N$-Benzyloxycarbonyl-2-aminoethyl $O$-(6-O-acetyl-2-azido-3,4-di- $O$-benzyl-}

2-deoxy- $\alpha$-D-glucopyranosyl)-(1 $\rightarrow 4)-O$-(methyl

3-O-benzyl-2-O-pivaloyl- $\alpha-\mathrm{L}-$ idopyranosyluronate)-(1 $\rightarrow 4)-O$-(6-O-acetyl-2-azido-3-O-benzyl-2-deoxy- $\alpha$-Dglucopyranosyl)-(1 $\rightarrow 4)$ - $O$-(methyl 3-O-benzyl-2-O-pivaloyl- $\alpha-\mathrm{L}-$ idopyranosyluronate)-(1 $\rightarrow 4)-O$-(6-O-acetyl-2-azido-3-O-benzyl-2-deoxy- $\alpha$-Dglucopyranosyl)-(1 $\rightarrow 4)$-methyl $\quad 3-O$-benzyl-2- $O$-pivaloyl- $\alpha$-L-idopyranosiduronate (20): $\mathrm{BF}_{3} \cdot \mathrm{Et}_{2} \mathrm{O}\left(145 \mu \mathrm{L}\right.$ of a $0.2 \mathrm{M}$ solution in dry $\left.\mathrm{CH}_{2} \mathrm{Cl}_{2}\right)$ was added under an argon atmosphere at room temperature to a mixture of $\mathbf{1 2}(30 \mathrm{mg}, 19 \mu \mathrm{mol})$ and $\mathbf{1 8}(29 \mathrm{mg}, 31$ $\mu \mathrm{mol})$ in dry $\mathrm{CH}_{2} \mathrm{Cl}_{2}(1.0 \mathrm{~mL})$. After stirring for $30 \mathrm{~min}$, the reaction mixture was neutralized with $\mathrm{Et}_{3} \mathrm{~N}$ and concentrated to dryness. The residue was purified by column chromatography (toluene-EtOAc 5:1 $\rightarrow 1: 1)$ to afford $\mathbf{2 0}(23 \mathrm{mg}, 52 \%)$, and unreacted $\mathbf{1 2}$ (8 mg, 27\%). TLC (toluene-EtOAc 4:1) $\mathrm{R}_{\mathrm{f}} 0.38 ;[\alpha]_{\mathrm{D}}^{20}+4^{\circ}\left(c 1.1, \mathrm{CHCl}_{3}\right) ;{ }^{1} \mathrm{H}-\mathrm{NMR}$ $\left(500 \mathrm{MHz}, \mathrm{CDCl}_{3}\right): \delta 7.39-7.25(\mathrm{~m}, 40 \mathrm{H}, \mathrm{Ar}), 5.37\left(\mathrm{~d}, 1 \mathrm{H}, J_{1,2}=6.0 \mathrm{~Hz}, \mathrm{H}-1 \mathrm{E}\right), 5.31(\mathrm{~d}$, $\left.1 \mathrm{H}, J_{1,2}=5.4 \mathrm{~Hz}, \mathrm{H}-1 \mathrm{C}\right), 5.15(\mathrm{bt}, 1 \mathrm{H}, \mathrm{NH}), 5.12\left(\mathrm{~d}, 1 \mathrm{H}, J_{1,2}=4.8 \mathrm{~Hz}, \mathrm{H}-1 \mathrm{~A}\right), 5.09$ (bs, 2H, $\left.\mathrm{CH}_{2}(\mathrm{Z})\right), 5.06-4.97$ (m, 5H, H-1B, H-1D, H-1F, H-2C, H-2E), 4.96-4.90 (m, 3H, H-2A, $\left.\mathrm{CH}_{2}(\mathrm{OBn})\right), 4.87-4.67\left(\mathrm{~m}, 11 \mathrm{H}, \mathrm{CH}_{2}(\mathrm{OBn})\right), 4.65\left(\mathrm{~d}, 1 \mathrm{H}, J_{4,5}=4.6 \mathrm{~Hz}, \mathrm{H}-5 \mathrm{~A}\right)$, 
4.61-4.56 (m, 2H, H-5C, $\left.\mathrm{CH}_{2}(\mathrm{OBn})\right), 4.49\left(\mathrm{~d}, 1 \mathrm{H}, J_{4,5}=5.6 \mathrm{~Hz}, \mathrm{H}-5 \mathrm{E}\right), 4.34-4.24(\mathrm{~m}$, $5 \mathrm{H}, \mathrm{H}-6 \mathrm{aB}, \mathrm{H}-6 \mathrm{bB}, \mathrm{H}-6 \mathrm{aD}, \mathrm{H}-6 \mathrm{bD}, \mathrm{H}-6 \mathrm{aF}), 4.15\left(\mathrm{dd}, 1 \mathrm{H}, J_{5,6}=3.3 \mathrm{~Hz}, J_{6 \mathrm{a}, 6 \mathrm{~b}}=12.2 \mathrm{~Hz}\right.$, H-6bF), $4.11\left(\mathrm{t}, 1 \mathrm{H}, J_{3,4}=5.3 \mathrm{~Hz}, \mathrm{H}-4 \mathrm{~A}\right), 4.08\left(\mathrm{t}, 1 \mathrm{H}, J_{3,4}=5.7 \mathrm{~Hz}, \mathrm{H}-4 \mathrm{C}\right), 4.03(\mathrm{t}, 1 \mathrm{H}$, $\left.J_{3,4}=6.2 \mathrm{~Hz}, \mathrm{H}-4 \mathrm{E}\right), 3.97-3.86$ (m, 8H, H-3A, H-3C, H-3E, H-4B, H-4D, H-5B, H-5D, H-5F), 3.84-3.78 (m, 2H, H-3F, $\left.\mathrm{CH}_{2}-\mathrm{O}\right), 3.75-3.69$ (m, 4H, H-3B or H-3D, COOMe), 3.66-3.58 (m, 8H, H-3B or H-3D, COOMe $\left.(3.64,3.61), \mathrm{CH}_{2}-\mathrm{O}\right), 3.53\left(\mathrm{t}, 1 \mathrm{H}, J_{3,4}=J_{4,5}=\right.$ $9.4 \mathrm{~Hz}, \mathrm{H}-4 \mathrm{~F}), 3.39$ (m, 2H, $\left.\mathrm{CH}_{2}-\mathrm{N}\right), 3.32-3.27$ (m, 3H, H-2B, H-2D, H-2F), 2.11, 2.07, $1.96\left(3 \mathrm{~s}, 9 \mathrm{H}, \mathrm{CH}_{3}(\mathrm{Ac})\right), 1.19$ (bs, 27H, $\left.\left(\mathrm{CH}_{3}\right)_{3}(\mathrm{Piv})\right) ;{ }^{13} \mathrm{C}-\mathrm{NMR}\left(125 \mathrm{MHz}, \mathrm{CDCl}_{3}\right)$ (Significant data from HSQC experiment): $\delta 99.0$ (C-1A), 98.1 (C-1B, C-1D, C-1F), 97.7 (C-1C), 97.5 (C-1E), 80.0 (C-3F), 79.9, 77.7 (C-3B, C-3D), 77.4 (C-4F), 76.8-73.5 (C-3A, C-3C, C-3E, C-4B, C-4D, C-4A, C-4C, C-4E, $\mathrm{CH}_{2}(\mathrm{OBn})$ ), 71.5 (C-2E, C-5E), 71.0 (C-2C, C-5C), 70.4 (C-2A), 70.3 (C-5A), 69.8 (C-5B, C-5D, C-5F), $68.4\left(\mathrm{CH}_{2}-\mathrm{O}\right)$, $66.7\left(\mathrm{CH}_{2}(\mathrm{Z})\right), 62.9$ (C-2B, C-2D, C-2F), 62.2 (C-6F), 61.8 (C-6B, C-6D), 52.3-52.0 (COOMe), $40.9\left(\mathrm{CH}_{2}-\mathrm{N}\right), 27.0\left(\left(\mathrm{CH}_{3}\right)_{3}(\mathrm{Piv})\right), 20.8\left(\mathrm{CH}_{3}(\mathrm{Ac})\right)$; ESI MS: m/z: calcd for $\mathrm{C}_{119} \mathrm{H}_{142} \mathrm{~N}_{10} \mathrm{O}_{39} \mathrm{Na}$ : 2357.9; found: $2357.7[M+\mathrm{Na}]^{+}$.

\section{2-aminoethyl 4-O-(2-deoxy-2-sulfamido-6-O-sulfo- $\alpha$-D-glucopyranosyl)-2-O-sulfo-} a-L-idopyranosiduronic acid (21): $\mathrm{H}_{2} \mathrm{O}_{2}(30 \%, 1.8 \mathrm{~mL})$ and a solution of $\mathrm{LiOH}(0.7$ $\mathrm{M}, 1.1 \mathrm{~mL})$ were added at $-5^{\circ} \mathrm{C}$ to a solution of $\mathbf{1 5}(43 \mathrm{mg}, 44 \mu \mathrm{mol})$ in $\mathrm{THF}(4.3 \mathrm{~mL})$. After stirring for $20 \mathrm{~h}$ at room temperature, $\mathrm{MeOH}(4.3 \mathrm{~mL})$ and an aqueous solution of $\mathrm{NaOH}(4 \mathrm{M}, 1.1 \mathrm{~mL})$ were added. After stirring for $20-24 \mathrm{~h}$ at room temperature, the reaction mixture was neutralized with a $4 \mathrm{M}$ solution of $\mathrm{HCl}$ and then diluted with $\mathrm{CH}_{2} \mathrm{Cl}_{2}(40 \mathrm{~mL})$ and washed with $\mathrm{H}_{2} \mathrm{O}(20 \mathrm{~mL})$. The organic phase was extracted with a solution of $\mathrm{Na}_{2} \mathrm{SO}_{3}(10 \%)$, dried $\left(\mathrm{MgSO}_{4}\right)$, filtered, and concentrated to give $\mathrm{N}$ benzyloxycarbonyl-2-aminoethyl 4-O-(2-azido-3,4-di- $O$-benzyl-2-deoxy- $\alpha$-D- 
glucopyranosyl)-3- $O$-benzyl- $\alpha$-L-idopyranosiduronic acid (30 mg, 82\%). ESI MS: $\mathrm{m} / z$ : calcd for $\mathrm{C}_{43} \mathrm{H}_{47} \mathrm{~N}_{4} \mathrm{O}_{13}$ : 827.3; found: $826.8[M-\mathrm{H}]^{-}$.

This compound (12 mg, $14 \mu \mathrm{mol}$ ), sulfur trioxide-trimethylamine complex (20 mg, 145 $\mu \mathrm{mol})$ and a magnetic stirrer bar were placed in a $5 \mathrm{~mL}$ microwave reaction vial and fitted with a septum, which was then pierced with a needle. The closed vial was then evacuated under high vacuum and left to dry for $12 \mathrm{~h}$. Argon was let in, dry DMF (1.0 $\mathrm{mL}$ ) was added and the reaction mixture was subjected to microwave radiation for 15 min at $100^{\circ} \mathrm{C}(80 \mathrm{~W}$ average power). The reaction vessel was cooled under a stream of nitrogen and quenched with $\mathrm{Et}_{3} \mathrm{~N}(200 \mu \mathrm{L}) . \mathrm{MeOH}(1 \mathrm{~mL})$ and $\mathrm{CH}_{2} \mathrm{Cl}_{2}(1 \mathrm{~mL})$ were added, and the solution was layered on the top of a Sephadex LH-20 chromatography column which was eluted with $\mathrm{CH}_{2} \mathrm{Cl}_{2} / \mathrm{MeOH}$ (1:1) to obtain $N$-benzyloxycarbonyl-2aminoethyl 4-O-(2-azido-3,4-di- $O$-benzyl-2-deoxy-6- $O$-sulfo- $\alpha$-D-glucopyranosyl)-3-Obenzyl-2- $O$-sulfo- $\alpha$-L-idopyranosiduronic acid as triethylammonium salt (17 mg, 91\%). TLC (EtOAc-Py-H $2 \mathrm{O}-\mathrm{AcOH}$ 10:5:3:1) $\mathrm{R}_{\mathrm{f}}$ 0.51; ${ }^{1} \mathrm{H}-\mathrm{NMR}$ (500 MHz, MeOD): $\delta$ 7.437.25 (m, $20 \mathrm{H}, \mathrm{Ar}), 5.18$ (bs, 1H, H-1A), 5.06 (m, 3H, H-1B, $\left.\mathrm{CH}_{2}(\mathrm{Z})\right)$, 4.90-4.65 (m, 7H, H-5A (4.74), $\left.\mathrm{CH}_{2}(\mathrm{OBn})\right), 4.49$ (bs, $\left.1 \mathrm{H}, \mathrm{H}-2 \mathrm{~A}\right), 4.33\left(\mathrm{dd}, 1 \mathrm{H}, J_{5,6 \mathrm{a}}=2.8 \mathrm{~Hz}, J_{6 \mathrm{a}, 6 \mathrm{~b}}=\right.$ $10.7 \mathrm{~Hz}, \mathrm{H}-6 \mathrm{aB}), 4.25$ (bt, 1H, H-3A), 4.20 (dd, 1H, J5,6b $=1.9 \mathrm{~Hz}, \mathrm{H}-6 \mathrm{bB}), 4.14$ (bt, 1H, H-4A), 3.97-3.93 (m, 2H, H-5B, H-3B), $3.80\left(\mathrm{~m}, 1 \mathrm{H}, \mathrm{CH}_{2}-\mathrm{O}\right), 3.66\left(\mathrm{t}, 1 \mathrm{H}, J_{3,4}=J_{4,5}\right.$ $=9.4 \mathrm{~Hz}, \mathrm{H}-4 \mathrm{~B}), 3.59\left(\mathrm{~m}, 1 \mathrm{H}, \mathrm{CH}_{2}-\mathrm{O}\right), 3.32\left(\mathrm{~m}, 3 \mathrm{H}, \mathrm{H}-2 \mathrm{~B}, \mathrm{CH}_{2}-\mathrm{N}\right), 3.19\left(\mathrm{q}, \mathrm{Et}_{3} \mathrm{NH}^{+}\right)$, $1.32\left(\mathrm{t}, \mathrm{Et}_{3} \mathrm{NH}^{+}\right) ;{ }^{13} \mathrm{C}-\mathrm{NMR}$ (125 MHz, MeOD) (Significant data from HSQC experiment): $\delta 99.4$ (C-1A), 96.5 (C-1B), 79.7 (C-3B), 77.6 (C-4B), 72.8 (C-3A), 71.9 (C-4A), 71.7 (C-2A), 70.2(C-5B), 67.1 (C-5A), $66.8\left(\mathrm{CH}_{2}-\mathrm{O}\right), 65.5$ (C-6B), 63.4 (C-2B); ESI MS: $m / z$ : calcd for $\mathrm{C}_{43} \mathrm{H}_{47} \mathrm{~N}_{4} \mathrm{O}_{19} \mathrm{~S}_{2}$ : 987.2 ; found: $986.7[M+2 \mathrm{H}]^{-}$; calcd for $\mathrm{C}_{61} \mathrm{H}_{94} \mathrm{~N}_{7} \mathrm{O}_{19} \mathrm{~S}_{2}:$ 1292.6; found: $1292.1\left[M+3 \mathrm{Et}_{3} \mathrm{NH}+\mathrm{H}\right]^{+}$. 
This compound (45 mg, $35 \mu \mathrm{mol})$ was dissolved in THF $(4.5 \mathrm{~mL})$ and treated with a $0.1 \mathrm{M}$ aqueous solution of $\mathrm{NaOH}(1.5 \mathrm{~mL})$. Then, a solution of $\mathrm{Me}_{3} \mathrm{P}$ in THF (139 $\mu \mathrm{L}$ of a $1 \mathrm{M}$ solution) was added and the reaction was stirred for $3 \mathrm{~h}$. The reaction mixture was neutralized with a $0.1 \mathrm{M}$ solution of $\mathrm{HCl}$ and concentrated to afford $\mathrm{N}$ benzyloxycarbonyl-2-aminoethyl 4 - $O$-(2-amino-3,4-di- $O$-benzyl-2-deoxy-6- $O$-sulfo- $\alpha$ D-glucopyranosyl)-3- $O$-benzyl-2- $O$-sulfo- $\alpha$-L-idopyranosiduronic acid as sodium salt. TLC (EtOAc-Py- $\left.\mathrm{H}_{2} \mathrm{O}-\mathrm{AcOH} \quad 10: 5: 3: 1\right) \quad \mathrm{R}_{\mathrm{f}} \quad 0.61$; $\quad$ ESI $\mathrm{MS}: \quad \mathrm{m} / \mathrm{z}$ : calcd for $\mathrm{C}_{43} \mathrm{H}_{48} \mathrm{~N}_{2} \mathrm{O}_{19} \mathrm{~S}_{2} \mathrm{Na}$ : 983.2; found: $982.7[M+\mathrm{Na}+\mathrm{H}]^{-}$; calcd for $\mathrm{C}_{43} \mathrm{H}_{48} \mathrm{~N}_{2} \mathrm{O}_{19} \mathrm{~S}_{2}$ : 480.1; found: $479.7[M+\mathrm{H}]^{2-}$; calcd for $\mathrm{C}_{43} \mathrm{H}_{49} \mathrm{~N}_{2} \mathrm{O}_{19} \mathrm{~S}_{2}$ : 961.2 ; found: $960.7[M+2 \mathrm{H}]^{-}$.

Triethylamine $(0.68 \mathrm{~mL})$ and then sulfur trioxide-pyridine complex $(56 \mathrm{mg}, 350$ $\mu$ mol $)$ were added to a solution of this disaccharide $(35 \mu \mathrm{mol})$ in anhydrous pyridine (3.0 mL). After stirring for $4 \mathrm{~h}$ at room temperature under an argon atmosphere, the reaction mixture was purified by Sephadex LH-20 chromatography $\left(\mathrm{CH}_{2} \mathrm{Cl}_{2} / \mathrm{MeOH}\right.$ 1:1) and RP-18 chromatography (10 mM AcOH-Et $3 \mathrm{~N}(\mathrm{pH} 7.0) / \mathrm{MeOH} 70: 30 \rightarrow 30: 70)$ to give $\mathrm{N}$-benzyloxycarbonyl-2-aminoethyl $\quad$ 4- $O$-(3,4-di- $O$-benzyl-2-deoxy-2-sulfamido-6- $O$ sulfo- $\alpha$-D-glucopyranosyl)-3- $O$-benzyl-2- $O$-sulfo- $\alpha$-L-idopyranosiduronic acid as triethylammonium salt. The corresponding sodium salt was obtained by elution from a column of Dowex 50WX4- $\mathrm{Na}^{+}$with $\mathrm{MeOH} / \mathrm{H}_{2} \mathrm{O}$ 9:1. TLC (EtOAc-Py- $\mathrm{H}_{2} \mathrm{O}-\mathrm{AcOH}$ 10:5:3:1) $\mathrm{R}_{\mathrm{f}}$ 0.29; ${ }^{1} \mathrm{H}-\mathrm{NMR}$ (500 MHz, MeOD) (data for triethylammonium salt): $\delta$ 7.46-7.21 (m, 20H, Ar), 5.45 (d, 1H, $\left.J_{1,2}=3.2 \mathrm{~Hz}, \mathrm{H}-1 \mathrm{~B}\right), 5.22$ (bs, 1H, H-1A), 5.14 (d, $\left.1 \mathrm{H}, \mathrm{CH}_{2}(\mathrm{OBn})\right), 5.03\left(\mathrm{~m}, 2 \mathrm{H}, \mathrm{CH}_{2}(\mathrm{Z})\right), 4.88-4.73$ (m, 6H, H-5A (4.74), $\mathrm{CH}_{2}(\mathrm{OBn})$ ), $4.55(\mathrm{~m}, 2 \mathrm{H}, \mathrm{H}-2 \mathrm{~A}, \mathrm{H}-3 \mathrm{~A}), 4.36\left(\mathrm{dd}, 1 \mathrm{H}, J_{5,6 \mathrm{a}}=2.1 \mathrm{~Hz}, J_{6 \mathrm{a}, 6 \mathrm{~b}}=10.7 \mathrm{~Hz}, \mathrm{H}-6 \mathrm{aB}\right), 4.24$ $\left(\mathrm{dd}, 1 \mathrm{H}, J_{5,6 \mathrm{~b}}=1.8 \mathrm{~Hz}, \mathrm{H}-6 \mathrm{bB}\right), 4.21(\mathrm{bs}, 1 \mathrm{H}, \mathrm{H}-4 \mathrm{~A}), 3.92(\mathrm{bd}, 1 \mathrm{H}, \mathrm{H}-5 \mathrm{~B}), 3.80(\mathrm{dd}, 1 \mathrm{H}$, $\left.J_{2,3}=10.4 \mathrm{~Hz}, J_{3,4}=9.4 \mathrm{~Hz}, \mathrm{H}-3 \mathrm{~B}\right), 3.76\left(\mathrm{~m}, 1 \mathrm{H}, \mathrm{CH}_{2}-\mathrm{O}\right), 3.62\left(\mathrm{t}, 1 \mathrm{H}, J_{4,5}=9.4 \mathrm{~Hz}, \mathrm{H}-\right.$ 4B), $3.55\left(\mathrm{~m}, 1 \mathrm{H}, \mathrm{CH}_{2}-\mathrm{O}\right), 3.44(\mathrm{dd}, 1 \mathrm{H}, \mathrm{H}-2 \mathrm{~B}), 3.35\left(\mathrm{~m}, 2 \mathrm{H}, \mathrm{CH}_{2}-\mathrm{N}\right), 3.19$ (q, 
$\mathrm{Et}_{3} \mathrm{NH}^{+}$), $1.30\left(\mathrm{t}, \mathrm{Et}_{3} \mathrm{NH}^{+}\right) ;{ }^{13} \mathrm{C}-\mathrm{NMR}(125 \mathrm{MHz}, \mathrm{MeOD})$ (Significant data from HSQC experiment): $\delta 102.1$ (C-1B), 100.5 (C-1A), 81.2 (C-3B), 78.5 (C-4B), 78.0 (C-4A), $76.1\left(\mathrm{C}-3 \mathrm{~A}, \mathrm{CH}_{2}(\mathrm{OBn})\right), 75.7\left(\mathrm{CH}_{2}(\mathrm{OBn})\right), 73.0\left(\mathrm{CH}_{2}(\mathrm{OBn})\right), 72.6(\mathrm{C}-2 \mathrm{~A}), 71.1$ (C5B), $67.9\left(\mathrm{CH}_{2}-\mathrm{O}\right), 67.7(\mathrm{C}-5 \mathrm{~A}), 67.2\left(\mathrm{CH}_{2}(\mathrm{Z})\right), 67.0(\mathrm{C}-6 \mathrm{~B}), 60.3$ (C-2B); ESI MS: $m / z$ : calcd for $\mathrm{C}_{43} \mathrm{H}_{46} \mathrm{~N}_{2} \mathrm{O}_{22} \mathrm{~S}_{3} \mathrm{NaK}$ : 550.1; found: $550.7[M+\mathrm{Na}+\mathrm{K}]^{2-}$.

A solution of this compound ( $35 \mu \mathrm{mol}$, as sodium salt) in $\mathrm{H}_{2} \mathrm{O} / \mathrm{MeOH}(2.7$ $\mathrm{mL} / 0.3 \mathrm{~mL}$ ) was hydrogenated in the presence of $\mathrm{Pd}(\mathrm{OH})_{2}$. After $24 \mathrm{~h}$, the suspension was filtered over Celite and concentrated. The residue was purified by Sephadex G-25 chromatography $\left(\mathrm{H}_{2} \mathrm{O} / \mathrm{MeOH}\right.$ 9:1) to give 21 after lyophilisation (15.3 mg, 60\% from $\mathrm{N}$-benzyloxycarbonyl-2-aminoethyl $\quad 4$ - $O$-(2-azido-3,4-di- $O$-benzyl-2-deoxy-6- $O$-sulfo$\alpha$-D-glucopyranosyl)-3-O-benzyl-2- $O$-sulfo- $\alpha$-L-idopyranosiduronic acid, 3 steps; $45 \%$ from 15, 5 steps, $85 \%$ average yield per step). ${ }^{1} \mathrm{H}-\mathrm{NMR}\left(500 \mathrm{MHz}, \mathrm{D}_{2} \mathrm{O}\right): \delta 5.46(\mathrm{~d}, 1 \mathrm{H}$, $\left.J_{1,2}=3.5 \mathrm{~Hz}, \mathrm{H}-1 \mathrm{~B}\right), 5.15\left(\mathrm{~d}, 1 \mathrm{H}, J_{1,2}=3.4 \mathrm{~Hz}, \mathrm{H}-1 \mathrm{~A}\right), 4.55\left(\mathrm{~d}, 1 \mathrm{H}, J_{4,5}=2.9 \mathrm{~Hz}, \mathrm{H}-5 \mathrm{~A}\right)$, $4.36\left(\mathrm{dd}, 1 \mathrm{H}, J_{5,6 \mathrm{a}}=3.1 \mathrm{~Hz}, J_{6 \mathrm{a}, 6 \mathrm{~b}}=11.2 \mathrm{~Hz}, \mathrm{H}-6 \mathrm{aB}\right), 4.33\left(\mathrm{dd}, 1 \mathrm{H}, J_{2,3}=6.5 \mathrm{~Hz}, \mathrm{H}-2 \mathrm{~A}\right)$, $4.23(\mathrm{~m}, 2 \mathrm{H}, \mathrm{H}-3 \mathrm{~A}, \mathrm{H}-6 \mathrm{bB}), 4.14\left(\mathrm{t}, 1 \mathrm{H}, J_{3,4}=3.3 \mathrm{~Hz}, \mathrm{H}-4 \mathrm{~A}\right), 4.04\left(\mathrm{~m}, 1 \mathrm{H}, \mathrm{CH}_{2}-\mathrm{O}\right)$, $4.00\left(\mathrm{dt}, 1 \mathrm{H}, J_{4,5}=9.8 \mathrm{~Hz}, \mathrm{H}-5 \mathrm{~B}\right), 3.82\left(\mathrm{~m}, 1 \mathrm{H}, \mathrm{CH}_{2}-\mathrm{O}\right), 3.64\left(\mathrm{t}, 1 \mathrm{H}, J_{2,3}=J_{3,4}=9.7 \mathrm{~Hz}\right.$, $\mathrm{H}-3 \mathrm{~B}), 3.57\left(\mathrm{t}, 1 \mathrm{H}, J_{4,5}=9.7 \mathrm{~Hz}, \mathrm{H}-4 \mathrm{~B}\right), 3.26\left(\mathrm{~m}, 3 \mathrm{H}, \mathrm{H}-2 \mathrm{~B}, \mathrm{CH}_{2}-\mathrm{N}\right) ;{ }^{13} \mathrm{C}-\mathrm{NMR}(125$ $\mathrm{MHz}, \mathrm{D}_{2} \mathrm{O}$ ) (Significant data from HMQC experiment): $\delta 99.3$ (C-1A), 96.4 (C-1B), 76.7 (C-2A), 75.7 (C-4A), 70.8 (C-3B), 69.7 (C-5B), 69.3 (C-5A), 69.2 (C-3A), 69.1 (C-4B), $66.5(\mathrm{C}-6 \mathrm{~B}), 64.1\left(\mathrm{CH}_{2}-\mathrm{O}\right), 57.7(\mathrm{C}-2 \mathrm{~B}), 39.2\left(\mathrm{CH}_{2}-\mathrm{N}\right)$; ESI MS: $m / z$ : calcd for $\mathrm{C}_{14} \mathrm{H}_{23} \mathrm{~N}_{2} \mathrm{O}_{20} \mathrm{~S}_{3} \mathrm{Na}_{2}:$ 680.98; found: $680.97[M+\mathrm{H}+2 \mathrm{Na}]^{-}$; calcd for $\mathrm{C}_{14} \mathrm{H}_{24} \mathrm{~N}_{2} \mathrm{O}_{20} \mathrm{~S}_{3} \mathrm{Na}$ : 659.00; found: $658.99[M+2 \mathrm{H}+\mathrm{Na}]^{-}$; calcd for $\mathrm{C}_{14} \mathrm{H}_{25} \mathrm{~N}_{2} \mathrm{O}_{20} \mathrm{~S}_{3}$ : 637.01; found: 637.01 $[M+3 \mathrm{H}]^{-}$.

2-aminoethyl $O$-(2-deoxy-2-sulfamido-6- $O$-sulfo- $\alpha$-D-glucopyranosyl)-(1 $\rightarrow 4)-O$-(2$O$-sulfo- $\alpha$-L-idopyranosyluronic acid)-(1 $\rightarrow 4)$ - $O$-(2-deoxy-2-sulfamido-6-O-sulfo- $\alpha$ - 
D-glucopyranosyl)-(1 $\rightarrow$ 4)-2-O -sulfo- $\alpha$-L-idopyranosiduronic acid (22): $\mathrm{H}_{2} \mathrm{O}_{2}(30 \%$, $0.44 \mathrm{~mL})$ and a solution of $\mathrm{LiOH}(0.7 \mathrm{M}, 0.27 \mathrm{~mL})$ were added at $-5^{\circ} \mathrm{C}$ to a solution of $19(18 \mathrm{mg}, 11 \mu \mathrm{mol})$ in THF $(1.2 \mathrm{~mL})$. After stirring for $20 \mathrm{~h}$ at room temperature, $\mathrm{MeOH}(1.2 \mathrm{~mL})$ and a solution of $\mathrm{NaOH}(4 \mathrm{M}, 0.28 \mathrm{~mL})$ were added. After stirring for 20-24 $\mathrm{h}$ at room temperature, the reaction mixture was neutralized with a $4 \mathrm{M}$ solution of $\mathrm{HCl}$ and then diluted with $\mathrm{CH}_{2} \mathrm{Cl}_{2}(20 \mathrm{~mL})$ and washed with $\mathrm{H}_{2} \mathrm{O}(10 \mathrm{~mL})$. The organic phase was extracted with a solution of $\mathrm{Na}_{2} \mathrm{SO}_{3}(10 \%)$, dried $\left(\mathrm{MgSO}_{4}\right)$, filtered, and concentrated to give $\mathrm{N}$-benzyloxycarbonyl-2-aminoethyl $O$-(2-azido-3,4-di- $O$ benzyl-

2-deoxy- $\alpha$-D-glucopyranosyl)-( $1 \rightarrow 4)-O$-(3-O-benzyl- $\alpha$-L-idopyranosyluronic $\quad$ acid)$(1 \rightarrow 4)-O$-(2-azido-3-O-benzyl-2-deoxy- $\alpha$-D-glucopyranosyl)-( $1 \rightarrow 4)$-3- $O$-benzyl- $\alpha$-Lidopyranosiduronic acid (12 mg, 80\%). ESI MS: m/z: calcd for $\mathrm{C}_{69} \mathrm{H}_{76} \mathrm{~N}_{7} \mathrm{O}_{23}: 1370.5$; found: 1369.9 [M-H] $]^{-}$calcd for $\mathrm{C}_{69} \mathrm{H}_{74} \mathrm{~N}_{7} \mathrm{O}_{23} \mathrm{Na}_{4}: 1460.4$; found: 1459.8 [M-3H+4Na] ${ }^{+}$. This compound $(12 \mathrm{mg}, 8.7 \mu \mathrm{mol})$ and sulfur trioxide-trimethylamine complex $(24 \mathrm{mg}$, $174 \mu \mathrm{mol})$ were dissolved in dry $\mathrm{DMF}(1.0 \mathrm{~mL})$ and heated at $100^{\circ} \mathrm{C}$ for $30 \mathrm{~min}$ using microwave radiation as described above. The reaction vessel was cooled and $\mathrm{Et}_{3} \mathrm{~N}$ (150 $\mu \mathrm{L}), \mathrm{MeOH}(1 \mathrm{~mL})$ and $\mathrm{CH}_{2} \mathrm{Cl}_{2}(1 \mathrm{~mL})$ were added. The solution was layered on the top of a Sephadex LH-20 chromatography column which was eluted with $\mathrm{CH}_{2} \mathrm{Cl}_{2} / \mathrm{MeOH}$ (1:1) to obtain $N$-benzyloxycarbonyl-2-aminoethyl $O$-(2-azido-3,4-di- $O$ benzyl-2-deoxy-6- $O$-sulfo- $\alpha$-D-glucopyranosyl)-( $1 \rightarrow 4$ )- $O$-(3- $O$-benzyl-2- $O$-sulfo- $\alpha$-Lidopyranosyluronic $\quad$ acid)-( $(1 \rightarrow 4)-O$-(2-azido-3-O-benzyl-2-deoxy-6- $O$-sulfo- $\alpha$-Dglucopyranosyl)-(1 $\rightarrow 4)-3-O$-benzyl-2- $O$-sulfo- $\alpha$-L-idopyranosiduronic $\quad$ acid as triethylammonium salt (19 $\mathrm{mg}, 95 \%)$. The corresponding sodium salt was obtained by elution from a column of Dowex 50WX4- $\mathrm{Na}^{+}$with $\mathrm{MeOH} / \mathrm{H}_{2} \mathrm{O}$ 3:1. TLC (EtOAc-Py$\mathrm{H}_{2} \mathrm{O}-\mathrm{AcOH}$ 10:5:3:1) $\mathrm{R}_{\mathrm{f}}$ 0.44; ${ }^{1} \mathrm{H}-\mathrm{NMR}$ (500 MHz, MeOD): $\delta$ 7.46-7.17 (m, 30H, Ar), 
5.49 (bs, 1H, H-1C), 5.22 (bs, 1H, H-1A), 5.10 (d, 1H, J1,2 = $3.7 \mathrm{~Hz}, \mathrm{H}-1 \mathrm{~B}), 5.06$ (bs, $\left.2 \mathrm{H}, \mathrm{CH}_{2}(\mathrm{Z})\right), 5.01\left(\mathrm{~d}, 1 \mathrm{H}, J_{1,2}=3.7 \mathrm{~Hz}, \mathrm{H}-1 \mathrm{D}\right), 5.00\left(\mathrm{~d}, 1 \mathrm{H}, J_{4,5}=1.5 \mathrm{~Hz}, \mathrm{H}-5 \mathrm{C}\right), 4.90-$ 4.64 (m, 10H, $\left.\mathrm{CH}_{2}(\mathrm{OBn}), \mathrm{H}-5 \mathrm{~A}(4.83)\right), 4.60$ (bs, 1H, H-2C), 4.52 (bs, 1H, H-2A), 4.42 (d, $\left.1 \mathrm{H}, \mathrm{CH}_{2}(\mathrm{OBn})\right), 4.35-4.31$ (m, 2H, H-6aB, H-6aD), 4.27-4.22 (m, 3H, H-3A, H-3C, H-6bB), 4.16 (bs, 1H, H-4A), 4.12 (bd, 1H, H-6bD), 4.05 (t, 1H, $J_{3,4}=J_{4,5}=9.6 \mathrm{~Hz}$, H-4B), 3.97 (m, 2H, H-4C, H-5B), 3.85 (m, 2H, H-3D, $\left.\mathrm{CH}_{2}-\mathrm{O}\right), 3.74$ (m, 2H, H-3B, H-5D), 3.67-3.60 (m, 2H, H-4D, $\mathrm{CH}_{2}-\mathrm{O}$ ), 3.47 (dd, 1H, $\left.J_{2,3}=10.3 \mathrm{~Hz}, \mathrm{H}-2 \mathrm{D}\right), 3.37$ (m, $\left.2 \mathrm{H}, \mathrm{CH}_{2}-\mathrm{N}\right), 3.29\left(\mathrm{dd}, 1 \mathrm{H}, J_{2,3}=10.3 \mathrm{~Hz}, \mathrm{H}-2 \mathrm{~B}\right) ;{ }^{13} \mathrm{C}-\mathrm{NMR}(125 \mathrm{MHz}, \mathrm{MeOD})$ (Significant data from HSQC experiment): $\delta 100.6$ (C-1A), 98.2 (C-1C), 96.6 (C-1D), 96.3 (C-1B), 81.5 (C-3D), 79.6 (C-3B), 78.5 (C-4D), 76.2, 76.1, 75.6, 73.1, 73.1 $\left(\mathrm{CH}_{2}(\mathrm{OBn})\right), 72.4$ (C-3A), 72.1 (C-2A, C-4B), 72.0 (C-4C), 71.7 (C-3C, C-4A), 71.3 (C-5B), 71.2 (C-5D), 70.7 (C-2C), $68.2\left(\mathrm{CH}_{2}-\mathrm{O}\right), 67.8$ (C-5A), 67.3 (C-5C), 67.2 $\left(\mathrm{CH}_{2}(\mathrm{Z})\right), 67.1(\mathrm{C}-6 \mathrm{~B}), 66.6(\mathrm{C}-6 \mathrm{D}), 65.3(\mathrm{C}-2 \mathrm{~B}), 65.0(\mathrm{C}-2 \mathrm{D}), 41.4\left(\mathrm{CH}_{2}-\mathrm{N}\right)$; ESI MS: $m / z$ : calcd for $\mathrm{C}_{69} \mathrm{H}_{73} \mathrm{~N}_{7} \mathrm{O}_{35} \mathrm{~S}_{4} \mathrm{~K}_{2}:$ 882.6; found: $882.4[M+2 \mathrm{H}+2 \mathrm{~K}]^{2-}$; calcd for $\mathrm{C}_{75} \mathrm{H}_{90} \mathrm{~N}_{8} \mathrm{O}_{35} \mathrm{~S}_{4}:$ 895.2; found: $894.9\left[M+3 \mathrm{H}+\mathrm{Et}_{3} \mathrm{NH}\right]^{2-}$; calcd for $\mathrm{C}_{69} \mathrm{H}_{71} \mathrm{~N}_{7} \mathrm{O}_{35} \mathrm{~S}_{4} \mathrm{~K}_{2} \mathrm{Na}_{2}$ : 904.6; found: $904.9[M+2 \mathrm{~K}+2 \mathrm{Na}]^{2-}$.

This compound (9 mg, $5 \mu \mathrm{mol})$ was dissolved in THF $(1.6 \mathrm{~mL})$ and treated with a $0.1 \mathrm{M}$ aqueous solution of $\mathrm{NaOH}(0.43 \mathrm{~mL})$. Then, a solution of $\mathrm{Me}_{3} \mathrm{P}$ in THF $(40 \mu \mathrm{L}$ of a $1 \mathrm{M}$ solution) was added and the reaction was stirred for $3 \mathrm{~h}$. The reaction mixture was neutralized with a $0.1 \mathrm{M}$ solution of $\mathrm{HCl}$ and concentrated to afford $\mathrm{N}$ benzyloxycarbonyl-2-aminoethyl $O$-(2-amino-3,4-di- $O$-benzyl-2-deoxy-6- $O$-sulfo- $\alpha$-D-

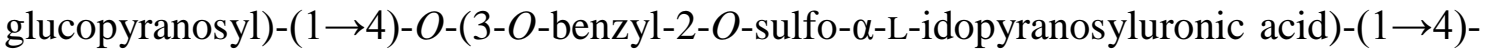
$O$-(2-amino-3-O-benzyl-2-deoxy-6- $O$-sulfo- $\alpha$-D-glucopyranosyl)-( $1 \rightarrow 4)-3$ - $O$-benzyl-2$O$-sulfo- $\alpha$-L-idopyranosiduronic acid as sodium salt. TLC (EtOAc-Py- $\mathrm{H}_{2} \mathrm{O}-\mathrm{AcOH}$ 
10:5:3:1) $\mathrm{R}_{\mathrm{f}}$ 0.43; ESI MS: $m / z$ : calcd for $\mathrm{C}_{69} \mathrm{H}_{79} \mathrm{~N}_{3} \mathrm{O}_{35} \mathrm{~S}_{4}: 818.7$; found: $818.1[M+4 \mathrm{H}]^{2-}$ ; calcd for $\mathrm{C}_{69} \mathrm{H}_{75} \mathrm{~N}_{3} \mathrm{O}_{35} \mathrm{~S}_{4} \mathrm{Na} 4$ : 862.6; found: $862.0[M+4 \mathrm{Na}]^{2-}$.

Triethylamine $(110 \mu \mathrm{L})$ and then sulfur trioxide-pyridine complex (16 mg, 98 $\mu \mathrm{mol})$ were added to a solution of this compound $(5 \mu \mathrm{mol})$ in anhydrous pyridine $(0.6$ $\mathrm{mL}$ ). After stirring for $6 \mathrm{~h}$ at room temperature under an argon atmosphere, the reaction mixture was purified by Sephadex LH-20 chromatography $\left(\mathrm{CH}_{2} \mathrm{Cl}_{2} / \mathrm{MeOH} 1: 1\right)$ and RP-18 chromatography (10 mM AcOH-Et $3 \mathrm{~N}(\mathrm{pH} 7.0) / \mathrm{MeOH} 90: 10 \rightarrow 0: 100)$ to give $N$ benzyloxycarbonyl-2-aminoethyl $O$-(3,4-di- $O$-benzyl-2-deoxy-2-sulfamido-6- $O$-sulfo$\alpha$-D-glucopyranosyl)-( $1 \rightarrow 4$ )- $O$-(3- $O$-benzyl-2- $O$-sulfo- $\alpha$-L-idopyranosyluronic $\quad$ acid)$(1 \rightarrow 4)-O$-(3-O-benzyl-2-deoxy-2-sulfamido-6- $O$-sulfo- $\alpha$-D-glucopyranosyl)-(1 $\rightarrow 4)-3$ $O$-benzyl-2- $O$-sulfo- $\alpha$-L-idopyranosiduronic acid as triethylammonium salt. The corresponding sodium salt was obtained by elution from a column of Dowex 50WX4$\mathrm{Na}^{+}$with $\mathrm{MeOH} / \mathrm{H}_{2} \mathrm{O}$ 9:1. TLC (EtOAc-Py- $\mathrm{H}_{2} \mathrm{O}-\mathrm{AcOH}$ 5:5:3:1) $\mathrm{R}_{\mathrm{f}}$ 0.57; ${ }^{1} \mathrm{H}-\mathrm{NMR}$ (500 MHz, MeOD): $\delta$ 7.52-7.05 (m, 30H, Ar), 5.99 (bs, 1H, H-1C), 5.36 (d, 1H, $J_{1,2}=3.6$ $\mathrm{Hz}, \mathrm{H}-1 \mathrm{D})$, 5.32-5.26 (m, 4H, H-1A (5.32), H-1B (5.30), $\left.\mathrm{CH}_{2}(\mathrm{OBn})\right), 5.03$ (m, 2H, $\left.\mathrm{CH}_{2}(\mathrm{Z})\right), 4.93$ (d, 1H, $\left.\mathrm{CH}_{2}(\mathrm{OBn})\right), 4.80$ (bs, 1H, H-5C), 4.77-4.71 (m, 6H, H-2A (4.74), H-2C (4.73), $\left.\mathrm{CH}_{2}(\mathrm{OBn})\right), 4.68\left(\mathrm{~d}, 1 \mathrm{H}, \mathrm{CH}_{2}(\mathrm{OBn})\right), 4.61(\mathrm{bs}, 1 \mathrm{H}, \mathrm{H}-5 \mathrm{~A}), 4.57$ (d, 1H, $\left.\mathrm{CH}_{2}(\mathrm{OBn})\right), 4.51-4.47$ (m, 2H, H-3A (4.50), $\left.\mathrm{CH}_{2}(\mathrm{OBn})\right), 4.41$ (m, 1H, H-5B), 4.37-4.32 (m, 3H, H-3C (4.36), H-6aD, H-6bD), 4.20-4.06 (m, 6H, H-4C (4.18), H-6aB (4.16), H4A (4.13), H-6bB (4.11), H-5D (4.09), H-3B (4.08)), 3.88 (t, 1H, $J_{3,4}=J_{4,5}=10.4 \mathrm{~Hz}$, $\mathrm{H}-4 \mathrm{~B}), 3.85\left(\mathrm{t}, 1 \mathrm{H}, J_{2,3}=J_{3,4}=9.8 \mathrm{~Hz}, \mathrm{H}-3 \mathrm{D}\right), 3.73\left(\mathrm{~m}, 1 \mathrm{H}, \mathrm{CH}_{2}-\mathrm{O}\right), 3.62-3.50(\mathrm{~m}, 4 \mathrm{H}$, H-4D (3.60), H-2B (3.56), H-2D (3.53), $\left.\mathrm{CH}_{2}-\mathrm{O}\right), 3.31$ (m, $\left.2 \mathrm{H}, \mathrm{CH}_{2}-\mathrm{N}\right) ;{ }^{13} \mathrm{C}-\mathrm{NMR}(125$ $\mathrm{MHz}, \mathrm{MeOD}$ ) (Significant data from HSQC experiment): $\delta$ 100.1 (C-1D), 100.0 (C-1A), 97.2 (C-1B), 94.4 (C-1C), 81.4 (C-3D), 78.8 (C-4D), 77.1 (C-4B), 76.4 (C-4C), 76.2, $76.0\left(\mathrm{CH}_{2}(\mathrm{OBn})\right), 75.9(\mathrm{C}-3 \mathrm{~B}), 75.5(\mathrm{C}-3 \mathrm{C}), 75.2\left(\mathrm{CH}_{2}(\mathrm{OBn})\right), 74.6(\mathrm{C}-4 \mathrm{~A}), 72.9$ 
(C-2A), 72.8, $72.6\left(\mathrm{CH}_{2}(\mathrm{OBn})\right), 71.7$ (C-3A), 71.5 (C-2C), 70.8 (C-5D), 69.6 (C-5C), $68.8(\mathrm{C}-5 \mathrm{~A}), 68.6(\mathrm{C}-5 \mathrm{~B}), 68.4(\mathrm{C}-6 \mathrm{~B}), 68.0\left(\mathrm{CH}_{2}-\mathrm{O}\right), 67.6(\mathrm{C}-6 \mathrm{D}), 67.3\left(\mathrm{CH}_{2}(\mathrm{Z})\right), 60.2$ (C-2B), 59.7 (C-2D), $41.3\left(\mathrm{CH}_{2}-\mathrm{N}\right)$; ESI MS: m/z: calcd for $\mathrm{C}_{69} \mathrm{H}_{73} \mathrm{~N}_{3} \mathrm{O}_{41} \mathrm{~S}_{6} \mathrm{Na}_{6}: 964.5$; found: $964.5[M+6 \mathrm{Na}]^{2-}$; calcd for $\mathrm{C}_{69} \mathrm{H}_{73} \mathrm{~N}_{3} \mathrm{O}_{41} \mathrm{~S}_{6} \mathrm{Na}_{10}$ : 1010.6; found: 1010.6 $[M+10 \mathrm{Na}]^{2+}$.

A solution of this compound $\left(5 \mu \mathrm{mol}\right.$, as sodium salt) in $\mathrm{H}_{2} \mathrm{O} / \mathrm{MeOH}(2.7$ $\mathrm{mL} / 0.3 \mathrm{~mL}$ ) was hydrogenated in the presence of $\mathrm{Pd}(\mathrm{OH})_{2}$. After $24 \mathrm{~h}$, the suspension was filtered over Celite and concentrated. The residue was purified by Sephadex G-25 chromatography $\left(\mathrm{H}_{2} \mathrm{O} / \mathrm{MeOH} 9: 1\right)$ to give 22 after lyophilisation $(3.2 \mathrm{mg}, 47 \%$ from the $O$-sulfated intermediate, 3 steps; $36 \%$ from 19,5 steps, $82 \%$ average yield per step). ${ }^{1} \mathrm{H}$ NMR (500 MHz, $\left.\mathrm{D}_{2} \mathrm{O}\right): \delta 5.47\left(\mathrm{~d}, 1 \mathrm{H}, J_{1,2}=3.6 \mathrm{~Hz}, \mathrm{H}-1 \mathrm{~B}\right), 5.39\left(\mathrm{~d}, 1 \mathrm{H}, J_{1,2}=3.5 \mathrm{~Hz}\right.$, H-1D), 5.21 (bd, 1H, H-1C), 5.08 (d, 1H, J1,2 = 4.1 Hz, H-1A), 4.82 (bd, 1H, H-5C), $4.53\left(\mathrm{~d}, 1 \mathrm{H}, J_{4,5}=3.2 \mathrm{~Hz}, \mathrm{H}-5 \mathrm{~A}\right), 4.40-4.25$ (m, 5H, H-2C (4.34), H-2A (4.31), H-6aB, H-6aD, H-6bB or H-6bD), 4.21-4.16 (m, 3H, H-3C, H-3A, H-6bB or H-6bD), 4.14 (t, $\left.1 \mathrm{H}, J_{3,4}=J_{4,5}=3.8 \mathrm{~Hz}, \mathrm{H}-4 \mathrm{~A}\right), 4.08(\mathrm{bt}, 1 \mathrm{H}, \mathrm{H}-4 \mathrm{C}), 4.05-3.97(\mathrm{~m}, 3 \mathrm{H}, \mathrm{H}-5 \mathrm{~B}, \mathrm{H}-5 \mathrm{D}$, $\left.\mathrm{CH}_{2}-\mathrm{O}\right), 3.81\left(\mathrm{~m}, 1 \mathrm{H}, \mathrm{CH}_{2}-\mathrm{O}\right), 3.76\left(\mathrm{t}, 1 \mathrm{H}, J_{3,4}=J_{4,5}=9.5 \mathrm{~Hz}, \mathrm{H}-4 \mathrm{~B}\right), 3.67-3.61(\mathrm{~m}, 2 \mathrm{H}$, H-3B, H-3D), $3.56\left(\mathrm{t}, 1 \mathrm{H}, J_{3,4}=J_{4,5}=9.6 \mathrm{~Hz}, \mathrm{H}-4 \mathrm{D}\right), 3.29-3.21(\mathrm{~m}, 4 \mathrm{H}, \mathrm{H}-2 \mathrm{~B}, \mathrm{H}-2 \mathrm{D}$, $\left.\mathrm{CH}_{2}-\mathrm{N}\right) ;{ }^{13} \mathrm{C}-\mathrm{NMR}\left(125 \mathrm{MHz}, \mathrm{D}_{2} \mathrm{O}\right)$ (Significant data from HSQC experiment): $\delta 99.6$ (C-1A), 99.1 (C-1C), 97.2 (C-1D), 95.9 (C-1B), 77.5 (C-2A), 76.1 (C-4B, C-4C), 75.9 (C-4A), 75.4 (C-2C), 69.9 (C-5A, C-3A, C-5D), 69.3 (C-3B, C-3D), 69.1 (C-5B, C-4D), 68.9 (C-5C), 68.5 (C-3C), 66.5, 66.3 (C-6B, C-6D), $64.4\left(\mathrm{CH}_{2}-\mathrm{O}\right), 58.0(\mathrm{C}-2 \mathrm{~B}), 57.8$ (C-2D), $39.4\left(\mathrm{CH}_{2}-\mathrm{N}\right)$; ESI MS: $m / z$ : calcd for $\mathrm{C}_{26} \mathrm{H}_{38} \mathrm{~N}_{3} \mathrm{O}_{39} \mathrm{~S}_{6} \mathrm{Na}_{5}$ : 661.45; found: $661.44[M+5 \mathrm{Na}+\mathrm{H}]^{2-} ; \quad$ calcd for $\mathrm{C}_{26} \mathrm{H}_{39} \mathrm{~N}_{3} \mathrm{O}_{39} \mathrm{~S}_{6} \mathrm{Na}_{4}: \quad 650.46 ;$ found: 650.45 $[M+4 \mathrm{Na}+2 \mathrm{H}]^{2-}$; calcd for $\mathrm{C}_{26} \mathrm{H}_{40} \mathrm{~N}_{3} \mathrm{O}_{39} \mathrm{~S}_{6} \mathrm{Na}_{3}: 639.47$; found: $639.46[M+3 \mathrm{Na}+3 \mathrm{H}]^{2-}$. 
2-aminoethyl $O$-(2-deoxy-2-sulfamido-4,6-di- $O$-sulfo- $\alpha$-D-glucopyranosyl)-( $1 \rightarrow 4$ )$O$-(2-O-sulfo- $\alpha$-L-idopyranosyluronic $\quad$ acid)-(1 $\rightarrow 4)-O$-(2-deoxy-2-sulfamido-6- $O$ sulfo- $\alpha$-D-glucopyranosyl)-( $1 \rightarrow 4)-O$-(2-O-sulfo- $\alpha$-L-idopyranosyluronic $\quad$ acid)$(1 \rightarrow 4)-O$-(2-deoxy-2-sulfamido-6- $O$-sulfo- $\alpha$-D-glucopyranosyl)-(1 $\rightarrow 4)-2-O$-sulfo- $\alpha$ L-idopyranosiduronic acid (23): $\mathrm{H}_{2} \mathrm{O}_{2}(30 \%, 1.4 \mathrm{~mL})$ and a solution of $\mathrm{LiOH}(0.7 \mathrm{M}$, $0.84 \mathrm{~mL})$ were added at $-5^{\circ} \mathrm{C}$ to a solution of $\mathbf{1 4}(76 \mathrm{mg}, 34 \mu \mathrm{mol})$ in $\mathrm{THF}(3.8 \mathrm{~mL})$. After stirring for $20 \mathrm{~h}$ at room temperature, $\mathrm{MeOH}(3.8 \mathrm{~mL})$ and a solution of $\mathrm{NaOH}(4$ $\mathrm{M}, 0.87 \mathrm{~mL}$ ) were added. After stirring for $20-24 \mathrm{~h}$ at room temperature, the reaction mixture was neutralized with a $4 \mathrm{M}$ solution of $\mathrm{HCl}$ and then diluted with $\mathrm{CH}_{2} \mathrm{Cl}_{2}(80$ $\mathrm{mL})$ and washed with $\mathrm{H}_{2} \mathrm{O}(40 \mathrm{~mL})$. The organic phase was extracted with a solution of $\mathrm{Na}_{2} \mathrm{SO}_{3}(10 \%)$, dried $\left(\mathrm{MgSO}_{4}\right)$, filtered, and concentrated to give the desired saponified hexasaccharide (60 mg, 97\%). ESI MS: $m / z$ : calcd for $\mathrm{C}_{88} \mathrm{H}_{96} \mathrm{~N}_{10} \mathrm{O}_{33} \mathrm{Na}_{3}$ : 1889.6; found: $1889.5[M+3 \mathrm{Na}-4 \mathrm{H}]^{-}$; calcd for $\mathrm{C}_{88} \mathrm{H}_{99} \mathrm{~N}_{10} \mathrm{O}_{33}: 1823.6$; found: $1823.5[M-\mathrm{H}]^{+}$.

This compound $(12 \mathrm{mg}, 6.7 \mu \mathrm{mol})$ and sulfur trioxide-trimethylamine complex $(33 \mathrm{mg}, 230 \mu \mathrm{mol})$ were dissolved in dry DMF $(1.0 \mathrm{~mL})$ and heated for $30 \mathrm{~min}$ at $100^{\circ} \mathrm{C}$ using microwaves as described above. The reaction vessel was cooled and $\mathrm{Et}_{3} \mathrm{~N}$ (150 $\mu \mathrm{L}), \mathrm{MeOH}(1 \mathrm{~mL})$ and $\mathrm{CH}_{2} \mathrm{Cl}_{2}(1 \mathrm{~mL})$ were added. The solution was layered on the top of a Sephadex LH-20 chromatography column which was eluted with $\mathrm{CH}_{2} \mathrm{Cl}_{2} / \mathrm{MeOH}$ (1:1) to obtain the desired hepta $O$-sulfated hexasaccharide as triethylammonium salt. The corresponding sodium salt was obtained by elution from a column of Dowex 50WX4- $\mathrm{Na}^{+}$with $\mathrm{MeOH} / \mathrm{H}_{2} \mathrm{O}$ 3:1. TLC (EtOAc-Py- $\mathrm{H}_{2} \mathrm{O}-\mathrm{AcOH}$ 5:5:3:1) $\mathrm{R}_{\mathrm{f}} 0.42 ;{ }^{1} \mathrm{H}-\mathrm{NMR}(500 \mathrm{MHz}, \mathrm{MeOD}): \delta$ 7.52-7.18 (m, 35H, Ar), 5.53 (bs, $1 \mathrm{H}$, H-1E), 5.47 (bs, 1H, H-1C), 5.22 (bs, 1H, H-1A), 5.21 (d, 1H, $\left.\mathrm{CH}_{2}(\mathrm{OBn})\right), 5.12$ (d, 1H, $J_{1,2}=3.7 \mathrm{~Hz}, \mathrm{H}-1 \mathrm{~B}$ or H-1D), $5.09\left(\mathrm{~d}, 1 \mathrm{H}, J_{1,2}=3.8 \mathrm{~Hz}, \mathrm{H}-1 \mathrm{~B}\right.$ or H-1D), 5.07 (bs, 3H, H-1F, $\mathrm{CH}_{2}(\mathrm{Z})$ ), 5.04 (bs, 1H, H-5C), 5.03 (bs, 1H, H-5E), 4.90-4.79 (m, 3H, $\mathrm{CH}_{2}(\mathrm{OBn})$, 
H-5A (4.82)), 4.77-4.59 (m, 7H, $\left.\mathrm{CH}_{2}(\mathrm{OBn}), \mathrm{H}-2 \mathrm{E}(4.62)\right), 4.53$ (bs, 2H, H-2C, H-2A), 4.50 (m, 2H, $\left.\mathrm{CH}_{2}(\mathrm{OBn}), \mathrm{H}-6 \mathrm{aF}\right), 4.49-4.42$ (m, 2H, $\left.\mathrm{CH}_{2}(\mathrm{OBn})\right), 4.36-4.15$ (m, 11H, H4F, H-6aB, H-6aD, H-3E, H-3C, H-3A, H-6bB, H-6bD, H-6bF, H-4A, H-4C), 4.07 (m, 2H, H-4B, H-4D), 4.03 (m, 1H, H-4E), 4.00 (m, 1H, H-5B or H-5D), 3.94 (m, 1H, H5F), 3.90-3.73 (m, 4H, H-3F, $\mathrm{CH}_{2}-\mathrm{O}, \mathrm{H}-5 \mathrm{~B}$ or H-5D, H-3B or H-3D), 3.71-3.60 (m, 2H, H-3B or H-3D, $\mathrm{CH}_{2}-\mathrm{O}$ ), 3.45-3.39 (m, 2H, H-2F, H-2B or H-2D), 3.37 (m, 2H, $\left.\mathrm{CH}_{2}-\mathrm{N}\right)$, $3.31(\mathrm{~m}, 1 \mathrm{H}, \mathrm{H}-2 \mathrm{~B}$ or $\mathrm{H}-2 \mathrm{D}) ;{ }^{13} \mathrm{C}-\mathrm{NMR}(125 \mathrm{MHz}, \mathrm{MeOD})$ (Significant data from HSQC experiment): $\delta 100.1$ (C-1A), 98.6 (C-1E), 98.5 (C-1C), 96.8, 96.1 (C-1B, C1D), 95.8 (C-1F), 80.2 (C-3F), 80.1, 79.7 (C-3B, C-3D), 78.0 (C-4F), 76.7, 76.5, 76.1, 73.5, $73.3\left(\mathrm{CH}_{2}(\mathrm{OBn})\right), 72.9$ (C-3A or C-3C), 72.7 (C-2A), 72.3 (C-4B, C-4D), 72.1 (C-4A), 71.5 (C-4E, C-2C), 71.4 (C-3E, C-3A or C-3C), 71.3, 71.2 (C-5B, C-5D), 71.1 (C-4C, C-5F), 70.9 (C-2E), $68.3\left(\mathrm{CH}_{2}-\mathrm{O}\right), 68.1$ (C-5A), 67.8 (C-6F), 67.5 (C-5C, C-5E), $67.4\left(\mathrm{CH}_{2}(\mathrm{Z}), \mathrm{C}-6 \mathrm{~B}, \mathrm{C}-6 \mathrm{D}\right), 65.9,65.3(\mathrm{C}-2 \mathrm{~B}, \mathrm{C}-2 \mathrm{D}), 64.5(\mathrm{C}-2 \mathrm{~F}), 41.5\left(\mathrm{CH}_{2}-\mathrm{N}\right) ; \mathrm{ESI}$ MS: $m / z$ : calcd for $\mathrm{C}_{142} \mathrm{H}_{237} \mathrm{~N}_{19} \mathrm{O}_{54} \mathrm{~S}_{7}: 1648.2$; found: $1648.3\left[M+9 \mathrm{Et}_{3} \mathrm{NH}+3 \mathrm{H}\right]^{2+}$.

This hexasaccharide $(6.7 \mu \mathrm{mol})$ was dissolved in THF $(2.4 \mathrm{~mL})$ and treated with a $0.1 \mathrm{M}$ aqueous solution of $\mathrm{NaOH}(0.96 \mathrm{~mL})$. Then, a solution of $\mathrm{Me}_{3} \mathrm{P}$ in THF $(88 \mu \mathrm{L}$ of a $1 \mathrm{M}$ solution) was added and the reaction was stirred for $6 \mathrm{~h}$. The reaction mixture was neutralized with a $0.1 \mathrm{M}$ solution of $\mathrm{HCl}$ and concentrated. $\mathrm{MeOH}(1 \mathrm{~mL}), \mathrm{CH}_{2} \mathrm{Cl}_{2}$ $(1 \mathrm{~mL})$ and $\mathrm{Et}_{3} \mathrm{~N}(250 \mu \mathrm{L})$ were added, and the solution was layered on the top of Sephadex LH-20 chromatography column which was eluted with $\mathrm{CH}_{2} \mathrm{Cl}_{2} / \mathrm{MeOH}$ (1:1) to obtain the corresponding intermediate. TLC (EtOAc-Py- $\mathrm{H}_{2} \mathrm{O}-\mathrm{AcOH}$ 8:5:3:1) $\mathrm{R}_{\mathrm{f}}$ 0.56; ESI MS: $m / z$ : calcd for $\mathrm{C}_{88} \mathrm{H}_{97} \mathrm{~N}_{4} \mathrm{O}_{54} \mathrm{~S}_{7} \mathrm{Na}_{10}: 2527.2$; found: $2527.2[M+10 \mathrm{Na}+\mathrm{H}]^{+}$; calcd for $\mathrm{C}_{88} \mathrm{H}_{97} \mathrm{~N}_{4} \mathrm{O}_{54} \mathrm{~S}_{7} \mathrm{Na} 8: 2481.2$; found: $2481.0[M+8 \mathrm{Na}+\mathrm{H}]^{-}$.

This hexasaccharide $(6.7 \mu \mathrm{mol})$ and sulfur trioxide-trimethylamine complex (31 $\mathrm{mg}, 219 \mu \mathrm{mol})$ were dissolved in dry DMF $(2.0 \mathrm{~mL})$ in a $5 \mathrm{~mL}$ microwave reaction vial 
under an argon atmosphere. $\mathrm{Et}_{3} \mathrm{~N}(250 \mu \mathrm{L})$ was added and the reaction mixture was subjected to microwave radiation for $30 \mathrm{~min}$ at $60^{\circ} \mathrm{C}$ (20 $\mathrm{W}$ average power). The reaction vessel was cooled under a stream of nitrogen. The reaction mixture was purified by Sephadex LH-20 chromatography $\left(\mathrm{CH}_{2} \mathrm{Cl}_{2} / \mathrm{MeOH}\right.$ 1:1) and RP-18 chromatography $(10 \mathrm{mM}$ AcOH-Et $3 \mathrm{~N}(\mathrm{pH} 7.0) /$ Acetonitrile $95: 5 \rightarrow 50: 50)$ to give the desired tri $\mathrm{N}$-sulfated hexasaccharide as triethylammonium salt. The corresponding sodium salt was obtained by elution from a column of Dowex $50 \mathrm{WX} 4-\mathrm{Na}^{+}$with $\mathrm{MeOH} / \mathrm{H}_{2} \mathrm{O}$ 3:1. TLC (EtOAc-Py- $\mathrm{H}_{2} \mathrm{O}-\mathrm{AcOH}$ 6:5:3:1) $\mathrm{R}_{\mathrm{f}}$ 0.21; ${ }^{1} \mathrm{H}-\mathrm{NMR}(500 \mathrm{MHz}$, MeOD): $\delta$ 7.59-7.06 (m, 35H, Ar), 6.05 (bs, 1H, H-1E), 6.01 (bs, 1H, H-1C), 5.35-5.30 (m, 6H, H-1A, H-1B, H-1D, H-1F, $\mathrm{CH}_{2}(\mathrm{OBn})$ ), 5.05 (m, 2H, $\mathrm{CH}_{2}(\mathrm{Z})$ ), 4.96-4.80 (m, 6H, H-5E (4.92), H-5C (4.81), $\left.\mathrm{CH}_{2}(\mathrm{OBn})\right), 4.77-4.42$ (m, 14H, H-5A (4.69), H-2E (4.67), H-2C (4.64), H-2A (4.53), H-5B, H-5D, H-6aF (4.52), H-6bF (4.45), $\mathrm{CH}_{2}(\mathrm{OBn})$ ), 4.35-4.03 (m, 15H, H-4C (4.27), H-4E (4.24), H-4F (4.24), H-4A (4.20), H-3C (4.20), H-3A (4.13), H-3E (4.13), H-3B, H-3D, H-3F, H-5F (4.05), H-6aB, H6bB, H-6aD, H-6bD), 3.95 (t, 1H, H-4B or H-4D), 3.80 (m, 1H, $\left.\mathrm{CH}_{2}-\mathrm{O}\right), 3.74$ (t, 1H, H4B or $\mathrm{H}-4 \mathrm{D}), 3.61-3.52$ (m, 4H, $\left.\mathrm{CH}_{2}-\mathrm{O}, \mathrm{H}-2 \mathrm{~B}, \mathrm{H}-2 \mathrm{D}, \mathrm{H}-2 \mathrm{~F}\right), 3.33$ (m, 2H, $\mathrm{CH}_{2}-\mathrm{N}$ ); ${ }^{13} \mathrm{C}-\mathrm{NMR}$ (125 MHz, MeOD) (Significant data from HSQC experiment): $\delta 99.5$ (C-1A), 96.8 (C-1B, C-1D, C-1F), 94.3 (C-1C), 94.2 (C-1E), 77.6 (C-4B or C-4D), 77.4 (C-4F), 77.1 (C-4B or C-4D), 75.4, 75.2, $74.9\left(\mathrm{CH}_{2}(\mathrm{OBn})\right), 74.3$ (C-3A, C-3E), 73.6-70.8 (C3C, C-3B, C-3D, C-3F, C-4A, C-4C (73.2), C-4E (71.8), C-2A (71.8), C-2C (72.4), C-2E (71.2), C-5B, C-5D, $\left.\mathrm{CH}_{2}(\mathrm{OBn})\right), 69.6$ (C-5C), 69.4 (C-5F), 69.1 (C-5E), 68.7 (C5A), 68.1 (C-6B or C-6D), 67.9 (C-6F), 67.7 (C-6B or C-6D), $67.3\left(\mathrm{CH}_{2}(\mathrm{Z})\right), 67.2$ $\left(\mathrm{CH}_{2}-\mathrm{O}\right), 60.1$ (C-2B, C-2D, C-2F), $42.0\left(\mathrm{CH}_{2}-\mathrm{N}\right)$; ESI MS: $\mathrm{m} / \mathrm{z}$ : calcd for $\mathrm{C}_{130} \mathrm{H}_{212} \mathrm{~N}_{11} \mathrm{O}_{63} \mathrm{~S}_{10} \mathrm{Na}: 1639.0$; found: $1639.2\left[M+7 \mathrm{Et}_{3} \mathrm{NH}+\mathrm{Na}+7 \mathrm{H}\right]^{2+}$; calcd for $\mathrm{C}_{142} \mathrm{H}_{243} \mathrm{~N}_{13} \mathrm{O}_{63} \mathrm{~S}_{10}$ : 1729.2; found: $1729.7\left[M+9 \mathrm{Et}_{3} \mathrm{NH}+6 \mathrm{H}\right]^{2+}$. 
A solution of this hexasaccharide $(6.7 \mu \mathrm{mol}$, as sodium salt $)$ in $\mathrm{H}_{2} \mathrm{O} / \mathrm{MeOH}(1.8$ $\mathrm{mL} / 0.2 \mathrm{~mL}$ ) was hydrogenated in the presence of $\mathrm{Pd}(\mathrm{OH})_{2}$. After $24 \mathrm{~h}$, the suspension was filtered over Celite and concentrated. The residue was purified by Sephadex G-25 chromatography $\left(\mathrm{H}_{2} \mathrm{O} / \mathrm{MeOH} 9: 1\right)$ to give $\mathbf{2 3}$ after lyophilisation $(4.4 \mathrm{mg}, 31 \%$ from the saponified intermediate, 4 steps; $30 \%$ from 14, 5 steps, $79 \%$ average yield per step). ${ }^{1} \mathrm{H}$ NMR (500 MHz, $\left.\mathrm{D}_{2} \mathrm{O}\right): \delta$ 5.50-5.47 (m, 2H, H-1F, H-1B or H-1D), $5.43\left(\mathrm{~d}, 1 \mathrm{H}, J_{1,2}=\right.$ $3.4 \mathrm{~Hz}, \mathrm{H}-1 \mathrm{~B}$ or H-1D), 5.27-5.21 (m, 2H, H-1C, H-1E), $5.12\left(\mathrm{~d}, 1 \mathrm{H}, J_{1,2}=3.8 \mathrm{~Hz}\right.$, H-1A), 4.84 (m, 2H, H-5C, H-5E), $4.56\left(\mathrm{~d}, 1 \mathrm{H}, J_{4,5}=3.1 \mathrm{~Hz}, \mathrm{H}-5 \mathrm{~A}\right), 4.48-4.26(\mathrm{~m}, 10 \mathrm{H}$, H-2A, H-2C, H-2E, H-6aB, H-6aD, H-6aF, H-6bB, H-6bD, H-6bF, H-4F), 4.24-4.19 (m, 3H, H-3A, H-3C, H-3E), 4.18-4.10 (m, 4H, H-4A, H-4C, H-4E, H-5F), 4.09-4.02 (m, 3H, H-5B, H-5D, $\mathrm{CH}_{2}-\mathrm{O}$ ), 3.87-3.76 (m, 4H, $\left.\mathrm{CH}_{2}-\mathrm{O}, \mathrm{H}-4 \mathrm{~B}, \mathrm{H}-4 \mathrm{D}, \mathrm{H}-3 \mathrm{~F}\right), 3.74-3.64$ (m, 2H, H-3B, H-3D), $3.36\left(\mathrm{dd}, 1 \mathrm{H}, J_{1,2}=3.3 \mathrm{~Hz}, J_{2,3}=10.8 \mathrm{~Hz}, \mathrm{H}-2 \mathrm{~F}\right), 3.33-3.21(\mathrm{~m}$, 4H, H-2B, H-2D, $\mathrm{CH}_{2}-\mathrm{N}$ ); ${ }^{13} \mathrm{C}-\mathrm{NMR}\left(125 \mathrm{MHz}, \mathrm{D}_{2} \mathrm{O}\right.$ ) (Significant data from HSQC experiment): $\delta 99.6(\mathrm{C}-1 \mathrm{~A}), 99.2$ (C-1C, C-1E), 96.4 (C-1B or C-1D), 95.9 (C-1F, C-1B or C-1D), 77.1 (C-2A, C-4F), 75.9 (C-4B, C-4D, C-4A, C-4C, C-4E, C-2C, C-2E), 69.9 (C-5A), 69.4 (C-3A, C-3C, C-3E, C-3B, C-3D, C-3F), 69.2 (C-5C, C-5E), 69.0 (C-5B, C-5D), 68.1 (C-5F), 66.4 (C-6B, C-6D, C-6F), $64.4\left(\mathrm{CH}_{2}-\mathrm{O}\right), 57.8$ (C-2B, C-2D), 57.4 (C-2F), $39.1\left(\mathrm{CH}_{2}-\mathrm{N}\right)$; ESI MS: $m / z$ : calcd for $\mathrm{C}_{38} \mathrm{H}_{51} \mathrm{~N}_{4} \mathrm{O}_{61} \mathrm{~S}_{10} \mathrm{Na}_{10}$ : 696.24; found: $696.22[M+10 \mathrm{Na}]^{3-}$.

2-aminoethyl $O$-(2-deoxy-2-sulfamido-6- $O$-sulfo- $\alpha$-D-glucopyranosyl)-(1 $\rightarrow 4)-O$-(2$O$-sulfo- $\alpha$-L-idopyranosyluronic acid)-(1 $\rightarrow 4)$ - $O$-(2-deoxy-2-sulfamido-6-O-sulfo- $\alpha$ D-glucopyranosyl)-(1 $\rightarrow 4)-O$-(2-O-sulfo- $\alpha$-L-idopyranosyluronic $\quad$ acid)-(1 $\rightarrow 4)-O$-(2deoxy-2-sulfamido-6-O-sulfo- $\alpha$-D-glucopyranosyl)-(1 $\rightarrow 4)-2-O$-sulfo- $\alpha$-Lidopyranosiduronic acid (24): $\mathrm{H}_{2} \mathrm{O}_{2}(30 \%, 0.64 \mathrm{~mL})$ and a solution of $\mathrm{LiOH}(0.7 \mathrm{M}$, $0.38 \mathrm{~mL})$ were added at $-5^{\circ} \mathrm{C}$ to a solution of $20(36 \mathrm{mg}, 15 \mu \mathrm{mol})$ in $\mathrm{THF}(1.7 \mathrm{~mL})$. 
After stirring for $20 \mathrm{~h}$ at room temperature, $\mathrm{MeOH}(1.7 \mathrm{~mL})$ and a solution of $\mathrm{NaOH}(4$ M, $0.39 \mathrm{~mL}$ ) were added. After stirring for $20-24 \mathrm{~h}$ at room temperature, the reaction mixture was neutralized with a $4 \mathrm{M}$ solution of $\mathrm{HCl}$ and then diluted with $\mathrm{CH}_{2} \mathrm{Cl}_{2}(20$ $\mathrm{mL})$ and washed with $\mathrm{H}_{2} \mathrm{O}(10 \mathrm{~mL})$. The organic phase was extracted with a solution of $\mathrm{Na}_{2} \mathrm{SO}_{3}(10 \%)$, dried $\left(\mathrm{MgSO}_{4}\right)$, and concentrated to give the desired saponified intermediate. ESI MS: $m / z$ : calcd for $\mathrm{C}_{95} \mathrm{H}_{102} \mathrm{~N}_{10} \mathrm{O}_{33} \mathrm{Na}_{3}$ : 1979.6; found: 1979.5 $[M+3 \mathrm{Na}-4 \mathrm{H}]^{-}$.

This compound $(15 \mu \mathrm{mol})$ and sulfur trioxide-trimethylamine complex (64 mg, $462 \mu \mathrm{mol})$ were dissolved in dry $\mathrm{DMF}(2.0 \mathrm{~mL})$ and heated for $30 \mathrm{~min}$ at $100^{\circ} \mathrm{C}$ using microwaves as described above. The reaction vessel was cooled and $\mathrm{Et}_{3} \mathrm{~N}(150 \mu \mathrm{L})$, $\mathrm{MeOH}(1 \mathrm{~mL})$ and $\mathrm{CH}_{2} \mathrm{Cl}_{2}(1 \mathrm{~mL})$ were added. The solution was layered on the top of a Sephadex LH-20 chromatography column which was eluted with $\mathrm{CH}_{2} \mathrm{Cl}_{2} / \mathrm{MeOH}(1: 1)$ to give the desired hexa $O$-sulfated hexasaccharide as triethylammonium salt. The corresponding sodium salt was obtained by elution from a column of Dowex 50WX4- $\mathrm{Na}^{+}$with $\mathrm{MeOH} / \mathrm{H}_{2} \mathrm{O}$ 3:1 (30 mg, 75\% from 20, 2 steps). TLC (EtOAc-Py$\mathrm{H}_{2} \mathrm{O}-\mathrm{AcOH}$ 8:5:3:1) $\mathrm{R}_{\mathrm{f}}$ 0.24; ${ }^{1} \mathrm{H}-\mathrm{NMR}(500 \mathrm{MHz}, \mathrm{MeOD}): \delta$ 7.42-7.16 (m, 40H, Ar), 5.93 (bs, 1H, H-1E), 5.82 (bs, 1H, H-1C), 5.23-5.10 (m, 4H, H-1F (5.21), H-1B or H1D, H-1A (5.16), $\left.\mathrm{CH}_{2}(\mathrm{OBn})\right), 5.09-4.88$ (m, 9H, H-1B or $\mathrm{H}-1 \mathrm{D}, \mathrm{CH}_{2}(\mathrm{Z}), \mathrm{CH}_{2}(\mathrm{OBn})$ ), 4.84-4.50 (m, 13H, H-5E, H-5C, H-2E (4.70), H-2C (4.65), H-5A (4.61), H-2A (4.52), $\mathrm{CH}_{2}(\mathrm{OBn})$ ), 4.41-3.98 (m, 16H, H-3E (4.39), H-6aB, H-6aD, H-6aF, H-3C (4.33), H-6bB, H-6bD, H-6bF, H-3A (4.27), H-4C, H-4E (4.11), H-4A (4.09), H-4B, H-4D, H-5B, H-5D), 3.89 (m, 1H, H-3F), 3.81-3.46 (m, 9H, H-3B, H-3D, $\mathrm{CH}_{2}-\mathrm{O}, \mathrm{H}-2 \mathrm{~F}$ (3.65), H-5F (3.64), H-4F (3.63), H-2B, H-2D), $3.36\left(\mathrm{~m}, 2 \mathrm{H}, \mathrm{CH}_{2}-\mathrm{N}\right) ;{ }^{13} \mathrm{C}-\mathrm{NMR}(125 \mathrm{MHz}$, MeOD) (Significant data from HSQC experiment): $\delta 100.6$ (C-1A), 95.4 (C-1C), 94.9 (C-1E), 94.7, 93.8 (C-1B, C-1D), 93.7 (C-1F), 82.5 (C-3F), 79.4 (C-4F), 78.8, 78.7 
(C-3B, C-3D), 76.0, 75.9, 75.6, $74.8\left(\mathrm{CH}_{2}(\mathrm{OBn})\right), 72.6$ (C-4A), 72.5, $72.4\left(\mathrm{CH}_{2}(\mathrm{OBn})\right)$, 71.3 (C-2C), 71.2 (C-2A), 71.1 (C-3A), 70.8-69.5 (C-4E, C-5F, C-2E, C-3E, C-3C, C-4C, C-4B, C-4D, C-5B, C-5D), 69.1 (C-5C, C-5E), 68.2 (C-5A), $67.6\left(\mathrm{CH}_{2}-\mathrm{O}\right), 67.2$ $\left(\mathrm{CH}_{2}(\mathrm{Z}), 66.7\right.$ (C-6B, C-6D, C-6F), $65.7(\mathrm{C}-2 \mathrm{~F}), 65.2(\mathrm{C}-2 \mathrm{~B}, \mathrm{C}-2 \mathrm{D}), 41.3\left(\mathrm{CH}_{2}-\mathrm{N}\right)$; ESI MS: $m / z$ : calcd for $\mathrm{C}_{95} \mathrm{H}_{97} \mathrm{~N}_{10} \mathrm{O}_{51} \mathrm{~S}_{6} \mathrm{Na}_{7}: 1273.1$; found: $1272.1[M+7 \mathrm{Na}]^{2-}$.

This $O$-sulfated hexasaccharide $(15 \mathrm{mg}, 5.8 \mu \mathrm{mol})$ was dissolved in THF (2.0 $\mathrm{mL})$ and treated with a $0.1 \mathrm{M}$ aqueous solution of $\mathrm{NaOH}(0.77 \mathrm{~mL})$. Then, a solution of $\mathrm{Me}_{3} \mathrm{P}$ in THF (70 $\mu \mathrm{L}$ of a $1 \mathrm{M}$ solution) was added and the reaction was stirred for $6 \mathrm{~h}$. The reaction mixture was neutralized with a $0.1 \mathrm{M}$ solution of $\mathrm{HCl}$ and concentrated to obtain the desired amino containing compound. TLC (EtOAc-Py- $\mathrm{H}_{2} \mathrm{O}-\mathrm{AcOH}$ 10:5:3:1) $\mathrm{R}_{\mathrm{f}}$ 0.34; ESI MS: $m / z$ : calcd for $\mathrm{C}_{95} \mathrm{H}_{103} \mathrm{~N}_{4} \mathrm{O}_{51} \mathrm{~S}_{6} \mathrm{Na}_{8}: 2491.3$; found: $2491.1[M+8 \mathrm{Na}]^{-}$; calcd for $\mathrm{C}_{95} \mathrm{H}_{103} \mathrm{~N}_{4} \mathrm{O}_{51} \mathrm{~S}_{6} \mathrm{Na}_{7}$ : 1234.2; found: $1234.0[M+7 \mathrm{Na}]^{2-}$.

This compound $(5.8 \mu \mathrm{mol})$ and sulfur trioxide-trimethylamine complex ( $25 \mathrm{mg}$, $174 \mu \mathrm{mol})$ were dissolved in dry DMF $(2.0 \mathrm{~mL})$ in a $5 \mathrm{~mL}$ microwave reaction vial under an argon atmosphere. $\mathrm{Et}_{3} \mathrm{~N}(200 \mu \mathrm{L})$ was added and the reaction mixture was subjected to microwave radiation for $30 \mathrm{~min}$ at $60^{\circ} \mathrm{C}(20 \mathrm{~W}$ average power). The reaction vessel was cooled under a stream of nitrogen. The reaction mixture was purified by Sephadex $\mathrm{LH}-20$ chromatography $\left(\mathrm{CH}_{2} \mathrm{Cl}_{2} / \mathrm{MeOH}\right.$ 1:1) to afford the corresponding tri $N$-sulfated hexasaccharide as triethylammonium salt. The sodium salt was obtained by elution from a column of Dowex 50WX4- $\mathrm{Na}^{+}$with $\mathrm{MeOH} / \mathrm{H}_{2} \mathrm{O}$ 3:1. TLC (EtOAc-Py- $\mathrm{H}_{2} \mathrm{O}-\mathrm{AcOH}$ 26:25:15:5) $\mathrm{R}_{\mathrm{f}} 0.26$.

A solution of this compound $\left(5.8 \mu \mathrm{mol}\right.$, as sodium salt) in $\mathrm{H}_{2} \mathrm{O} / \mathrm{MeOH}(1.35$ $\mathrm{mL} / 0.15 \mathrm{~mL}$ ) was hydrogenated in the presence of $\mathrm{Pd}(\mathrm{OH})_{2}$. After $24 \mathrm{~h}$, the suspension was filtered over Celite and concentrated. The residue was purified by Sephadex G-25 chromatography $\left(\mathrm{H}_{2} \mathrm{O} / \mathrm{MeOH} 9: 1\right)$ to give $\mathbf{2 4}$ after lyophilisation $(2.0 \mathrm{mg}, 17 \%$ from the 
$O$-sulfated intermediate, 3 steps; $13 \%$ from 20, 5 steps, $66 \%$ average yield per step). ${ }^{1} \mathrm{H}$ NMR (500 MHz, D $2 \mathrm{O}): \delta 5.48$ (bd, 1H, H-1B or H-1D), 5.44 (bd, 1H, H-1F), 5.39 (bs, 1H, H-1B or H-1D), 5.34 (bs, 1H, H-1C or H-1E), 5.25 (bs, 1H, H-1C or H-1E), 5.11 (bd, 1H, H-1A), 4.90-4.83 (m, 2H, H-5E, H-5C), 4.55 (bd, 1H, H-5A), 4.46-3.99 (m, 19H, H-6aB, H-6aD, H-6aF, H-6bB, H-6bD, H-6bF, H-2E, H-2C, H-2A (4.32), H-3A, H-3C, H-3E, H-4A (4.15), H-4C, H-4E, H-5B, H-5D, $\mathrm{CH}_{2}-\mathrm{O}$ (4.03), H-5F (4.01)), 3.873.77 (m, 3H, H-4B, H-4D, $\mathrm{CH}_{2}-\mathrm{O}$ (3.82)), 3.75-3.63 (m, 3H, H-3B, H-3D, H-3F (3.66)), $3.58\left(\mathrm{t}, 1 \mathrm{H}, J_{3,4}=J_{4,5}=9.6 \mathrm{~Hz}, \mathrm{H}-4 \mathrm{~F}\right), 3.33-3.18\left(\mathrm{~m}, 5 \mathrm{H}, \mathrm{H}-2 \mathrm{~B}, \mathrm{H}-2 \mathrm{D}, \mathrm{H}-2 \mathrm{~F}, \mathrm{CH}_{2}-\mathrm{N}\right.$

(3.24)); ${ }^{13} \mathrm{C}-\mathrm{NMR}$ (125 MHz, $\mathrm{D}_{2} \mathrm{O}$ ) (Significant data from HSQC experiment): $\delta 99.5$ (C-1A), 99.2, 98.9 (C-1C, C-1E), 96.9 (C-1B or C-1D), 96.7 (C-1F), 95.9 (C-1B or C1D), 77.7 (C-2A), 76.1 (C-4B or C-4D), 75.9 (C-4B or C-4D, C-4A, C-4C, C-4E, C-2C or C-2E), 75.2 (C-2C or C-2E), 70.9 (C-3F), 69.9 (C-3A, C-5A), 69.7 (C-5F), 69.5 (C-5C, C-5E, C-3B, C-3D), 69.2 (C-4F), 69.1 (C-5B, C-5D), 68.7, 68.5 (C-3C, C-3E), 66.4, 66.3 (C-6B, C-6D, C-6F), $64.5\left(\mathrm{CH}_{2}-\mathrm{O}\right), 57.8$ (C-2B, C-2D, C-2F), $39.3\left(\mathrm{CH}_{2}-\mathrm{N}\right)$. ESI MS: $m / z$ : calcd for $\mathrm{C}_{38} \mathrm{H}_{52} \mathrm{~N}_{4} \mathrm{O}_{58} \mathrm{~S}_{9} \mathrm{Na9}$ : 662.26; found: $662.24[M+9 \mathrm{Na}]^{3-}$; calcd for $\mathrm{C}_{38} \mathrm{H}_{53} \mathrm{~N}_{4} \mathrm{O}_{58} \mathrm{~S}_{9} \mathrm{Na}$ : 654.94 ; found: $654.92[M+8 \mathrm{Na}+\mathrm{H}]^{3-}$; calcd for $\mathrm{C}_{38} \mathrm{H}_{54} \mathrm{~N}_{4} \mathrm{O}_{58} \mathrm{~S}_{9} \mathrm{Na}_{7}$ : 647.61; found: $647.59[M+7 \mathrm{Na}+2 \mathrm{H}]^{3-}$.

\section{Acknowledgements}

We thank the CSIC (Grant 201180E021), the Spanish Ministry of Science and Innovation (Grant CTQ2009-07168), Junta de Andalucía (Grant P07-FQM-02969, and "Incentivo a Proyecto Internacional") and the European Union (FEDER support and Marie Curie Reintegration Grant) for financial support. J. A. acknowledges financial support from the MICINN through the Ramón y Cajal program.

\section{References and notes}


1. R. Raman, V. Sasisekharan and R. Sasisekharan, Chem. Biol., 2005, 12, $267-$ 277.

2. I. Capila and R. J. Linhardt, Angew. Chem. Int. Ed., 2002, 41, 391-412.

3. C. I. Gama and L. C. Hsieh-Wilson, Curr. Opin. Chem. Biol., 2005, 9, 609-619.

4. J. Kreuger, D. Spillmann, J. P. Li and U. Lindahl, J. Cell Biol., 2006, 174, 323327.

5. A. K. Powell, E. A. Yates, D. G. Fernig and J. E. Turnbull, Glycobiology, 2004, 14, 17R-30R.

6. J. R. Bishop, M. Schuksz and J. D. Esko, Nature, 2007, 446, 1030-1037.

7. M. Petitou and C. A. A. van Boeckel, Angew. Chem. Int. Ed., 2004, 43, 31183133.

8. $\quad$ L. Poletti and L. Lay, Eur. J. Org. Chem., 2003, 2999-3024.

9. C. Noti and P. H. Seeberger, Chem. Biol., 2005, 12, 731-756.

10. J. Angulo, R. Ojeda, J. L. de Paz, R. Lucas, P. M. Nieto, R. M. Lozano, M. Redondo-Horcajo, G. Giménez-Gallego and M. Martín-Lomas, ChemBioChem, 2004, 5, 55-61.

11. C. I. Gama, S. E. Tully, N. Sotogaku, P. M. Clark, M. Rawat, N. Vaidehi, W. A. Goddard, A. Nishi and L. C. Hsieh-Wilson, Nat. Chem. Biol., 2006, 2, 467-473.

12. D. M. Ratner, E. W. Adams, M. D. Disney and P. H. Seeberger, ChemBioChem, 2004, 5, 1375-1383.

13. J. L. de Paz and P. H. Seeberger, Mol. BioSyst., 2008, 4, 707-711.

14. J. L. de Paz, C. Noti and P. H. Seeberger, J. Am. Chem. Soc., 2006, 128, 27662767.

15. J. L. de Paz, C. Noti, F. Bohm, S. Werner and P. H. Seeberger, Chem. Biol., 2007, 14, 879-887.

16. R. Raman and R. Sasisekharan, Chem. Biol., 2007, 14, 873-874.

17. M. Rawat, C. I. Gama, J. B. Matson and L. C. Hsieh-Wilson, J. Am. Chem. Soc., 2008, 130, 2959-2961.

18. C. A. A. van Boeckel and M. Petitou, Angew. Chem. Int. Ed., 1993, 32, 16711690.

19. J. L. de Paz, J. Angulo, J. M. Lassaletta, P. M. Nieto, M. Redondo-Horcajo, R. M. Lozano, G. Giménez-Gallego and M. Martín-Lomas, ChemBioChem, 2001, 2, 673-685.

20. C. Noti, J. L. de Paz, L. Polito and P. H. Seeberger, Chem. Eur. J., 2006, 12, 8664-8686.

21. S. Arungundram, K. Al-Mafraji, J. Asong, F. E. Leach, I. J. Amster, A. Venot, J. E. Turnbull and G. J. Boons, J. Am. Chem. Soc., 2009, 131, 17394-17405.

22. F. Baleux, L. Loureiro-Morais, Y. Hersant, P. Clayette, F. Arenzana-Seisdedos, D. Bonnaffé and H. Lortat-Jacob, Nat. Chem. Biol., 2009, 5, 743-748.

23. A. Lubineau, H. Lortat-Jacob, O. Gavard, S. Sarrazin and D. Bonnaffé, Chem. Eur. J., 2004, 10, 4265-4282.

24. J. D. C. Codée, B. Stubba, M. Schiattarella, H. S. Overkleeft, C. A. A. van Boeckel, J. H. van Boom and G. A. van der Marel, J. Am. Chem. Soc., 2005, 127, 3767-3773.

25. J. C. Lee, X. A. Lu, S. S. Kulkarni, Y. S. Wen and S. C. Hung, J. Am. Chem. Soc., 2004, 126, 476-477.

26. H. A. Orgueira, A. Bartolozzi, P. Schell, R. E. J. N. Litjens, E. R. Palmacci and P. H. Seeberger, Chem. Eur. J., 2003, 9, 140-169.

27. J. Tatai and P. Fugedi, Tetrahedron, 2008, 64, 9865-9873. 
28. J. Chen, Y. Zhou, C. Chen, W. Xu and B. Yu, Carbohydr. Res., 2008, 343, 2853-2862.

29. J. L. de Paz and M. Martín-Lomas, Eur. J. Org. Chem., 2005, 1849-1858.

30. Y. P. Hu, S.Y. Lin, C.Y. Huang, M. M. L. Zulueta, J.Y. Liu, W. Chang and S. C. Hung, Nat. Chem., 2011, 3, 557-563.

31. G. Tiruchinapally, Z. Yin, M. El-Dakdouki, Z. Wang and X. Huang, Chem. Eur. J., 2011, 17, 10106-10112.

32. C. M. Dreef-Tromp, H. A. M. Willems, P. Westerduin, P. vanVeelen and C. A. A. van Boeckel, Bioorg. Med. Chem. Lett., 1997, 7, 1175-1180.

33. R. Ojeda, J. L. de Paz and M. Martín-Lomas, Chem. Commun., 2003, 24862487.

34. R. Ojeda, O. Terentí, J. L. de Paz and M. Martín-Lomas, Glycoconjugate J., 2004, 21, 179-195.

35. P. Czechura, N. Guedes, S. Kopitzki, N. Vázquez, M. Martín-Lomas and N. C. Reichardt, Chem. Commun., 2011, 47, 2390-2392.

36. Z. Wang, Y. Xu, B. Yang, G. Tiruchinapally, B. Sun, R. Liu, S. Dulaney, J. Liu and X. Huang, Chem. Eur. J., 2010, 16, 8365-8375.

37. T. Polat and C. H. Wong, J. Am. Chem. Soc., 2007, 129, 12795-12800.

38. S. Maza, J. L. de Paz and P. M. Nieto, Tetrahedron Lett., 2011, 52, 441-443.

39. R. Ojeda, J. L. de Paz, M. Martín-Lomas and J. M. Lassaletta, Synlett, 1999, 1316-1318.

40. J. L. de Paz, R. Ojeda, N. Reichardt and M. Martín-Lomas, Eur. J. Org. Chem., 2003, 3308-3324.

41. N. C. Reichardt and M. Martín-Lomas, ARKIVOC, 2005, 133-145.

42. Alternatively, $\mathbf{1 5}$ was obtained by glycosylation of disaccharide $\mathbf{1 8}$ with $\mathrm{N}$ benzyloxycarbonyl aminoethanol in good yield.

43. J. Kiss, Adv. Carbohydr. Chem. Biochem., 1974, 29, 229-303.

44. R. R. Schmidt and A. Toepfer, Tetrahedron Lett., 1991, 32, 3353-3356.

45. R. Lucas, D. Hamza, A. Lubineau and D. Bonnaffé, Eur. J. Org. Chem., 2004, 2107-2117.

46. D. Crich and V. Dudkin, J. Am. Chem. Soc., 2001, 123, 6819-6825.

47. L. Liao and F. I. Auzanneau, Org. Lett., 2003, 5, 2607-2610.

48. J. L. Hendel, A. Cheng and F. I. Auzanneau, Carbohydr. Res., 2008, 343, 29142923.

49. J. L. Hendel, J. W. Wang, T. A. Jackson, K. Hardmeier, R. De Los Santos and F. I. Auzanneau, J. Org. Chem., 2009, 74, 8321-8331.

50. A. Wang and F. I. Auzanneau, Carbohydr. Res., 2010, 345, 1216-1221.

51. R. Karlsson and A. Sjodin, US Pat., 0241724 A1, 2004.

52. S. Faham, R. E. Hileman, J. R. Fromm, R. J. Linhardt and D. C. Rees, Science, 1996, 271, 1116-1120.

53. M. Maccarana, B. Casu and U. Lindahl, J. Biol. Chem., 1993, 268, 2389823905.

54. L. Pellegrini, Curr. Opin. Struct. Biol., 2001, 11, 629-634 and references cited therein.

55. P. H. Liang, S. K. Wang and C. H. Wong, J. Am. Chem. Soc., 2007, 129, $11177-$ 11184.

56. S. Park and I. Shin, Org. Lett., 2007, 9, 1675-1678.

57. S. Cochran, C. Li, J. K. Fairweather, W. C. Kett, D. R. Coombe and V. Ferro, J. Med. Chem., 2003, 46, 4601-4608 and references cited therein. 